University of Louisville

ThinkIR: The University of Louisville's Institutional Repository

Electronic Theses and Dissertations

$12-2016$

\title{
Identification of residual descending pathways after human spinal cord injury.
}

Darryn A. Atkinson

University of Louisville

Follow this and additional works at: https://ir.library.louisville.edu/etd

Part of the Systems and Integrative Physiology Commons

\section{Recommended Citation}

Atkinson, Darryn A., "Identification of residual descending pathways after human spinal cord injury." (2016). Electronic Theses and Dissertations. Paper 2591.

https://doi.org/10.18297/etd/2591

This Doctoral Dissertation is brought to you for free and open access by ThinkIR: The University of Louisville's Institutional Repository. It has been accepted for inclusion in Electronic Theses and Dissertations by an authorized administrator of ThinkIR: The University of Louisville's Institutional Repository. This title appears here courtesy of the author, who has retained all other copyrights. For more information, please contact thinkir@louisville.edu. 


\title{
IDENTIFICATION OF RESIDUAL DESCENDING PATHWAYS AFTER HUMAN SPINAL CORD INJURY
}

By

\author{
Darryn A. Atkinson \\ B.S., Harding University, 1999 \\ M.P.T., University of Texas Southwestern Medical Center, 2003 \\ M.S., University of Louisville, 2014
}

\begin{abstract}
A Dissertation
Submitted to the Faculty of the

School of Medicine of the University of Louisville

in Partial Fulfillment of the Requirements

for the Degree of
\end{abstract}

Doctor of Philosophy in Anatomical Science and Neurobiology

Department of Anatomical Sciences and Neurobiology

University of Louisville

Louisville, Kentucky

December 2016 
Copyright 2016 by Darryn A. Atkinson

All rights reserved 



\title{
IDENTIFICATION OF RESIDUAL DESCENDING PATHWAYS AFTER HUMAN SPINAL CORD INJURY
}

\author{
By \\ Darryn A. Atkinson \\ B.S., Harding University, 1999 \\ M.P.T., University of Texas Southwestern Medical Center, 2003 \\ M.S., University of Louisville, 2014 \\ A Dissertation Approved on
}

December 1, 2016

By the following Dissertation Committee:

Dissertation Director- Dr. Susan J. Harkema

Dr. Andrea Behrman

Dr. Dena R. Howland

Dr. Charles H. Hubscher

Dr. David S.K. Magnuson

Dr. Jeffrey C. Petruska 


\section{DEDICATION}

This dissertation is dedicated to

My wife, Kim

My children, Kyley, Molly, Brandon, and Brooklyn

My parents, Ms. Martha A. Atkinson and Mr. Darryl L. Atkinson

My grandparents, Mr. Billy Joe Gunter and Ms. Marjorie Gunter

for their love, support, and encouragement. 


\section{ACKNOWLEDGEMENTS}

I would like to express my most sincere gratitude to my mentor, Dr. Susan Harkema for initially encouraging me to pursue my research interests at the University of Louisville, as well as for providing me with a wonderful graduate training experience. My achievements and the completion of this work were made possible by her support and mentorship. I would also like to express my respect and appreciation for my committee members Drs. Andrea Behrman, Dena Howland, Charles Hubscher, David Magnuson, and Jeffrey Petruska, who have generously given of their time and expertise. Each made unique and meaningful contributions to this project. Just as important, I need to thank each of the individuals who gave of their time and volunteered to participate in these studies-without them this work could not be completed. I also would like to acknowledge all current and former lab members, of which there are many. In particular, I would like to specifically acknowledge Dr. Dimitry Sayenko, Amber Mink, Erin Wyles, Danny Bryant, and Carie Tolfo for their contributions, encouragement, and support. Without their excellent mentorship, technical assistance, and expertise I would not be able to complete this work. I would also like to acknowledge to Frazier Rehab Institute and Kentucky One Health, Kentucky Spinal Cord \& Head Injury Research Trust Grant no. 11-7, National 
Institute of General Medical Sciences Grant 8 P30 GM-103507, Helmsley Foundation Grant \#2011PG-MED011, and the Commonwealth of Kentucky Challenge of Excellence Trust Fund their support of this research project. 


\section{ABSTRACT \\ IDENTIFICATION OF RESIDUAL DESCENDING PATHWAYS AFTER HUMAN SPINAL CORD INJURY}

Darryn A. Atkinson

December 1, 2016

Spinal Cord Injury (SCl) in humans is a heterogeneous diagnosis, resulting in variable paralysis and paresthesia based on the mechanism, rostrocaudal location, and severity of injury. Both neurophysiological and anatomical studies have suggested that subclinical residual supraspinal-spinal connectivity exists in a subset of individuals deemed to have motor and sensory complete injuries. Recent reports of volitional movement in chronic, motor complete individuals during epidural spinal stimulation have provided compelling evidence that these residual projections may be capable of mediating volitional movement when the functional state of spinal circuitry is electrically modulated.

I. It was the goal of this project to identify subclinical and pathwayspecific subliminal influences on spinal excitability after human $\mathrm{SCl}$, and further to determine their relationship to volitional muscle activation after injury.

II. Results demonstrated that volitional muscle activation can be identified and quantified in an objective manner via neurophysiological assessment, with greater resolution than current clinical measures. Electrophysiological studies 
probing the nervous system in subjects with chronic $\mathrm{SCl}$, evidence was obtained of residual descending influence even in subjects classified as having motor and sensory complete injuries. Comparisons between volitional muscle activation and detection of descending modulation of multisegmental muscle responses in incomplete SCI participants revealed that interlimb modulation of a given motor pool was a strong predictor of predicted volitional movement, but was also observed in muscles without volitional activation, suggesting the pathways mediating the observed modulation may be necessary but not sufficient for volitional muscle activation after SCI. 


\section{TABLE OF CONTENTS}

PAGE

ACKNOWLEDGEMENTS

ABSTRACT

iv

LIST OF TABLES

LIST OF FIGURES

GENERAL INTRODUCTION

NEUROPHYSIOLOGICAL ASSESSMENT OF MOTOR FUNCTION AFTER SPINAL CORD

INJURY

PATHWAY-SPECIFIC INVESTIGATION OF DESCENDING NEURAL INFLUENCE ON SPINAL MOTOR EXCITABILITY

MULTISEGMENTAL MUSCL RESPONSES

3

PROPRIOSPINAL TRACT ASSESSMENT

4

DEVELOPMENT OF METHODOLOGIES FOR IDENTIFICATION OF DESCENDING

INFLUENCE ON LUMBOSACRAL MOTOR EXCITABILITY

FUNCTIONAL NEUROPHYSIOLOGICAL ASSESSMENT (FNPA) 13

BACKGROUND

PROTOCOL DEVELOPMENT

VOLITIONAL MUSCLE ACTIVATION AFTER SCI

DEVELOPMENT OF NEUROPHYSIOLOCIAL PATHWAYS ASSESSMENT 21

BACKGROUND

CHARACTERIXATION OF MMR ACTIVATION PATTERNS EVOKED BY

TRANSCUTANEOUS SPINAL CORD STIMULATION

DESCENDING INTERLIMB MODULATION OF LUMBOSACRAL NETWORK EXCITABILITY IN HUMAN

43

INTRODUCTION

EXPERIMENTAL PROCEDURES

PARTICIPANTS

ELECTROMYOGRAPHY

45

SOLEUS H-REFLEX

45

MULTISEGMENTAL MUSCLE RESPONSES (MMRS)

46

ULNAR NERVE STIMULATION

47

EXPERIMENTAL PROTOCOLS

48

DATA ANALYSIS

49

RESULTS

ULNAR NERVE STIMULATION MODULATES THE AMPLITUDE OF SOLEUS

H-REFLEX AND MMR

ULNAR NERVE STIMULATION-INDUCED MODULATION OF BILATERAL LEG

MMRS

MAGNITUDE OF CONDITIONING EFFECT WAS RELATED TO RATE OF

RECRUITMENT 


\begin{tabular}{|c|c|}
\hline DISCUSSION & 52 \\
\hline DESCENDING INTERLIMB MODULATION OF LEG MMRS & 53 \\
\hline \multicolumn{2}{|c|}{ NEURAL PATHWAYS MEDIATING DESCENDING MODULATION OF SPINAL MOTOR } \\
\hline EXCITABILITY & 53 \\
\hline \multicolumn{2}{|l|}{ MAGNITUDE OF MMR MODULATION IS DEPENDENT UPON RATE OF } \\
\hline RECRUITMENT & 55 \\
\hline OPTIMIZATION OF STIMULATION LOCATION & 57 \\
\hline LIMITATIONS & 57 \\
\hline FUTURE DIRECTIONS & 58 \\
\hline CONCLUSIONS & 59 \\
\hline \multicolumn{2}{|c|}{ INTERLIMB REFLEXES REVEAL DESCENDING MODULATION OF SPINAL EXCITAILITY } \\
\hline AFTER HUMAN SPINAL CORD INJURY & 67 \\
\hline INTRODUCTION & 67 \\
\hline METHODS & 69 \\
\hline PARTICIPANTS & 69 \\
\hline FUNCTIONAL NEUROPHYSIOLOGICAL ASSESSMENT (FNPA) & 70 \\
\hline MULTISEGMENTAL MUSCLE RESPONSES (MMRS) & 71 \\
\hline ULNAR NERVE STIMULATION & 72 \\
\hline EXPERIMENTAL PROCEDURES & 72 \\
\hline DATA ANALYSIS & 73 \\
\hline RESULTS & 74 \\
\hline DISCUSSION & 77 \\
\hline PATHWAYS MEDIATING INTERLIMB MMR MODULATION & 78 \\
\hline \multicolumn{2}{|c|}{ MMR FACILITATION AND VOLITIONAL ACTIVATION WITHIN LUMBOSACRAL MOTOR } \\
\hline POOLS & 80 \\
\hline CONCLUSIONS & 82 \\
\hline DISCUSSION & 92 \\
\hline \multicolumn{2}{|c|}{ DESCENDING INTERLIMB MODULATION OF MMRS IN NEUROLOGICALLY INTACT } \\
\hline PARTICIPANTS & 97 \\
\hline DESCENDING INTERLIMB MODULATION OF MMRS AFTER SCI & 101 \\
\hline \multicolumn{2}{|c|}{ VOLUNTARY MUSCLE ACTIVATION AND INTERLIMB MMR FACILITATION AFTER SPINAL } \\
\hline CORD INJURY & 103 \\
\hline LIMITATIONS & 105 \\
\hline FUTURE DIRECTIONS & 106 \\
\hline REFERENCES & 111 \\
\hline CURRICULUM VITA & 133 \\
\hline
\end{tabular}




\section{LIST OF TABLES}

TABLE

TABLE 1

TABLE 2

TABLE 3

TABLE 4

TABLE 5

TABLE 6

TABLE 7

TABLE 8
PAGE

28

29

30

31

83

84

86

90 


\section{LIST OF FIGURES}

FIGURE

PAGE

FIGURE 1

32

FIGURE 2

FIGURE 3

FIGURE 4

FIGURE 5

FIGURE 6

FIGURE 7

FIGURE 8

FIGURE 9

FIGURE 10

FIGURE 11

62

FIGURE 12

63

FIGURE 13

64

FIGURE 14

65

FIGURE 15

66

FIGURE 16

85

FIGURE 17

87

FIGURE 18

88

FIGURE 19

89

FIGURE 20

91 


\section{CHAPTER I}

\section{GENERAL INTRODUCTION}

Recent studies of epidural spinal stimulation after human spinal cord injury $(\mathrm{SCl})$ have revealed that individuals with chronic, motor complete $\mathrm{SCl}$ may retain the capacity for volitional movement below the level of injury in the presence of epidural stimulation (Harkema, Gerasimenko et al. 2011, Angeli, Edgerton et al. 2014). In the first 3 participants in whom volitional movement ability was assessed at initiation of epidural spinal stimulation, volitional leg movement was observed between 4 and 11 days post stimulation onset (Angeli, Edgerton et al. 2014). One possible interpretation of these findings is that supraspinal centers retain some residual neural connectivity to the lumbosacral spinal circuitry, which is only detectable when the functional state of the spinal circuitry is modulated. Anatomical studies have demonstrated the existence of translesional axons in as many as $58 \%$ of spinal cord injured individuals who had been clinically classified as motor and sensory complete (Bunge, Puckett et al. 1993, Kakulas 1999, Kakulas and Kaelan 2015). Similarly, neurophysiological studies have demonstrated that even those individuals clinically diagnosed with motor and sensory complete injuries often retain some ability to modulate spinal motor neuron excitability below the level of injury, also possibly indicating the existence 
of residual descending fibers (Dimitrijevic, Dimitrijevic et al. 1984, Dimitrijevic, Hsu et al. 1992, McKay, Lim et al. 2004). It was the goal of this project to implement novel neurophysiological techniques for the identification spared and subliminal descending tracts, and to investigate the importance/relevance of these pathways to voluntary movement following human SCl.

There is mounting evidence to suggest that spinal electrical stimulation has the potential to significantly improve recovery of neurologic function after motor and sensory complete SCl. This population is typically limited to compensatory strategies and assistive devices to regain some measure of independence following injury. Improved understanding of the specific neural pathways and mechanisms underlying voluntary movement observed during epidural stimulation is critical to the continued development and refinement of epidural stimulation paradigms for translation to broader clinical populations. Moreover, knowledge of specific pathways mediating functional connectivity between spinal networks on either side of a spinal lesion may provide new targets for the development of novel recovery strategies and improve characterization of injury type and severity, allowing more individualized prognosis and therapeutic intervention selection by clinicians.

\section{Neurophysiological Assessment of Motor Function after Spinal Cord Injury}

Neurophysiologic assessment of voluntary and reflex muscle activation patterns following neurologic injury via multi-muscle electromyographic (EMG) analysis has been under investigation for over 35 years. (Dimitrijevic et al., 1980, Dimitrijevic et al., 1992a, Sherwood et al., 1996b, McKay et al., 2011a).This 
method takes advantage of the ability to monitor multiple muscles simultaneously during the performance of a motor task as well as during segmental reflex testing. Patterns of multi-muscle electromyographic (EMG) activity during both voluntary movement and reflex tests in individuals with $\mathrm{SCl}$ often exhibit cocontraction and/or spread of activity to distant spinal segments (Sherwood et al., 1996b, McKay et al., 2011b), suggesting altered descending control in many subjects who do retain some ability to voluntarily initiate movement after SCl. These studies led to the observation that, under specific standardized testing conditions, suprasegmental influences could be seen on segmental motor activity below the level of $\mathrm{SCl}$ in individuals with no ability to voluntarily activate segmental motor units below the level of lesion. (Dimitrijevic et al., 1977b, Dimitrijevic et al., 1984a, Sherwood et al., 1992) This has led to usage of the term "discomplete" to further characterize individuals given the clinical designation of motor complete SCl. A large number of individuals with clinically complete lesions (AIS A-B) have since been studied by this group; a majority were found to retain some ability to modulate spinal motor excitability below the level of injury (Dimitrijevic et al., 1992a, McKay et al., 2004). Although increased objectivity and sensitivity in the detection of volitional activation of motor units has been demonstrated (McKay et al., 2011b, Li et al., 2012), the specific neuronal pathways underlying modulation of spinal motor excitability after $\mathrm{SCl}$ cannot be identified with this method of assessment.

\section{Pathway-Specific Investigation of Descending Neural Influence on Spinal Motor}

\section{Excitability}


Despite the discussed neurophysiologic and anatomical evidence of translesional neural pathways after $\mathrm{SCl}$, identification of pathway-specific residual and/or de novo descending translesional connectivity in descending systems other than the corticospinal tracts are understudied. The H-reflex has been used to investigate descending supraspinal and intraspinal influence on lumbosacral motor excitability in non-injured human subjects, including corticospinal (Nielsen, 1995, Wolfe, 1996, Goulart et al., 2000, Guzman-Lopez et al., 2012), vestibulospinal (Kennedy et al., 2004, Lowery and Bent, 2009), reticulospinal (Ilic et al., 2011), and long propriospinal (Meinck, 1976, PiesiurStrehlow and Mienck, 1980, Meinck and Piesiur-Strehlow, 1981a, Delwaide and Crenna, 1984b, Frigon et al., 2004, Zehr et al., 2004) tracts. The H-reflex is advantageous for the study of paralyzed muscles which cannot be volitionally activated, and allows observation of both facilitative and inhibitory effects. Taskdependent modulation of the H-reflex via supra and intra-spinal inputs has been extensively studied, leading to the acceptance of the $\mathrm{H}$-reflex as being under descending influence (Delwaide and Crenna, 1984b, Capaday and Stein, 1986, Crone and Nielsen, 1989, Dyhre-Poulsen and Simonsen, 2002, Solopova and Selionov, 2012).

\section{Multisegmental Muscle Responses}

Single, brief electrical pulses over the posterior aspect of the lumbosacral spinal cord evoke short-latency compound motor action potentials in bilateral leg muscles (Dimitrijevic et al., 1983, Maertens de Noordhout et al., 1988), termed multi-segmental muscle responses (MMRs) (Courtine et al., 2007), posterior root 
muscle responses (Minassian et al., 2004), or sensory root evoked responses (Roy et al., 2012). Neurophysiological examinations of the effect of stimulation at various superior-inferior and anterior-posterior locations relative to the cord (Dimitrijevic et al., 1983) as well as observations of post activation depression suggest that dorsal root afferents are preferentially stimulated via transcutaneous stimulation, with the cathode positioned at midline over the dorsal aspect of the spinal cord (Minassian et al., 2004, Courtine et al., 2007, Hofstoetter et al., 2008, Roy et al., 2012). Modeling studies have also suggested large diameter posterior root afferents at the site of root entry into the cord will have the lowest thresholds in response to stimulation, with anterior root thresholds, and eventually superficial dorsal columns fiber thresholds being reached at successively higher intensities (Rattay et al., 2000, Ladenbauer et al., 2010a, Danner et al., 2011). Multiple investigators have investigated the modulatory properties of MMRs in order to further elucidate the nature of these responses. MMRs have been shown to share several properties with the peripherally evoked $\mathrm{H}$-reflex: MMRs are similarly inhibited by tendon vibration (Courtine et al., 2007, Minassian et al., 2007, Dy et al., 2010), the presence of reciprocal inhibition between antagonists can be observed (Minassian et al., 2007, Hofstoetter et al., 2008), and MMRs are facilitated during voluntary agonist contraction (Minassian et al., 2007, Hofstoetter et al., 2008). Further, task- and phase- dependent modulation during standing and gait also was observed (Courtine et al., 2007, Hofstoetter et al., 2008, Dy et al., 2010). These observations all support the segmental reflex nature of the response, suggesting activation of the la afferents at threshold 
stimulation intensities, reminiscent of the H-reflex evoked when stimulating the mixed nerve peripherally or intrathecally (Magladery et al., 1951b, Schieppati, 1987).

For the current project, methods were developed to take advantage of the ability to evoke MMRs from multiple bilateral segments within the lumbosacral enlargement using spinal stimulation to investigate residual neural influence of descending propriospinal pathways on the lumbosacral circuitry after SCI. MMRs address some of the limitations inherent to H-reflex modulation studies. These include difficulties evoking the reflex in some muscles due to difficulty locating the peripheral nerve, antidromic collision with increasing intensity of stimulation, and reflex stability issues during studies involving movement-due to changes in the relative position of the peripheral nerve being stimulated during limb movements (Zehr, 2002, Pierrot-Deseilligny, 2005), making MMRs advantageous for reflex testing.

The most important advantage of MMRs is the simultaneous detection of segmental responses in multiple bilateral leg muscles, representing the rostrocaudal extent of the lumbosacral enlargement. In contrast, the H-reflex methodology typically allows examination of one unilateral muscle at a time. Commonly, the soleus muscle is used; as such only the spinal segments innervating the soleus- S1 and S2 (Sharrard, 1964a, Kendall et al., 1983)- may be monitored. Comparison of the H-reflex and MMR modulation studies during standing (Hofstoetter et al., 2008) and ambulation in both healthy (Courtine et al., 
2007) and spinal cord injured subjects (Dy et al., 2010) illustrates this key benefit to use of MMRs when probing the spinal motor circuitry.

\section{Propriospinal Tract Assessment}

While human studies in $\mathrm{SCl}$ have primarily focused on corticospinal tract function (Wolfe, 1996, McKay et al., 1997, Curt et al., 1998, Curt et al., 2004, Curt and Ellaway, 2012), animal models of SCI have provided evidence that long descending propriospinal pathways can participate in synaptic plasticity, forming so-called detour pathways which re-establish functional cortical connections with the lumbosacral motor circuitry, ultimately leading to recovery of motor function (Bareyre et al., 2004, Courtine et al., 2009, Lang et al., 2012, Filli et al., 2014). Long propriospinal pathways have been extensively studied in animal models, and include long ascending and descending propriospinal neurons which reciprocally connect the cervical and lumbar enlargements (Jankowska et al., 1974, Schomburg et al., 1978, Beaumont et al., 2006, Reed et al., 2006, Ni et al., 2014). These findings have inspired new interest in descending propriospinal neurons as potential therapeutic targets following SCI (Cowley et al., 2010, Flynn et al., 2011).

Long descending propriospinal neurons projecting both ipsi- and contralaterally in the rat and the cat have been shown to receive substantially greater synaptic input from reticulospinal axon terminals as compared to corticospinals. (Matsuyama, Mori et al. 2004, Mitchell, McCallum et al. 2016). 
However, evidence suggests that mammals have increased numbers of corticospinal projections onto cervical propriospinal neurons coincident with increased hand dexterity, with humans having the most abundant cervical corticospinal projections (Lemon 2008). Interestingly, although very few published studies can be found which attempt to describe the location and relative number of descending axons at various rostro-caudal locations in the human spinal cord, one of the more recent of such studies concluded that below the cervical segments, a majority of descending fibers are propriospinal, with a majority of those being short propriospinal, and the next most common descending fiber type was reticulospinal(Nathan, Smith et al. 1996). Propriospinal axons mostly entered the gray matter by $L 2$, whereas reticulospinal fibers, which were more diffusely scattered throughout the spinal cord, descended to at least the lower lumbar levels.

C3-C4 propriospinals revceive converging descending input from all major descending pathways, including corticospinal, reticulospinal, vestibulospinal, and tectospinal contacts, in addition to contacts from peripheral upper limb afferents (Alstermark, Isa et al. 2007). The C3-C4 propriospinals project directly onto upper limb motor neurons, and have been shown to play a role in upper limb volitional motor control in cats, monkeys (Alstermark, Isa et al. 2007, Alstermark, Pettersson et al. 2011), and in parallel with direct cortico-motoneuronal inputs humans (Pierrot-Deseilligny 1989, Pierrot-Deseilligny 2002). However direct cortico-motoneuronal projections appear to be absent in rats (Alstermark, Ogawa et al. 2004). C3-C4 proppriospinals also project to long descending propriospinal 
neurons (Alstermark, Isa et al. 2007) located throughout the cervical enlargement (Bareyre, Kerschensteiner et al. 2004, Brockett, Seenan et al. 2013).

While some studying corticospinal pathways have suggested that the increase in cortico-motoneuronal control purported to exist in humans may be associated with a concomitant decrease in indirect contributions to upper limb motor control (Lemon 2008), the evidence for this is lacking. In response to this assertion, it has been pointed out that even though the number of corticospinal projections increases from cat to monkey to human, a majority of these projections still terminate in the in the intermediate gray matter or more dorsally, as opposed to direct spinal motor neuron synapses (Isa, Ohki et al. 2007).

The precise role of direct cortico-motoneuronal synaspes in motor control in comparison with indirect corticospinal influence via spinal interneurons and propriospinal neurons is still largely undetermined. Studies of corticospinal transections in both man and monkey resulted in significant and 'wellcoordinated' functional recovery of motor function (Bucy, Keplinger et al. 1964, Bucy, Ladpli et al. 1966), while transections of the anterior cord in humans generally produced little to no deficits in motor function (Nathan 1994), suggesting either ventral or dorsal descending motor systems is sufficient to enable functional motor control in the absence of the other in these species. It has been suggested that in humans, the nervous system might preferentially utilize the direct motoneuronal pathway for fine motor control, whereas the indirect cortico-propriospinal, cortico-reticulospinal (and potentially corticoreticulo-propriospinal) pathways are engaged when coordination of the upper, 
axial and lower limb musculature is needed as during ambulation, allowing taskspecific activation of spinal networks for interlimb coordination (Dietz and Michel 2009). This hypothesis is consistent with the known roles of propriospinal neurons in rats and cats (Ballion, Morin et al. 2001, Juvin, Simmers et al. 2005).

Reticulospinal fibers have also been shown to receive monosynaptic cortical inputs (Matsuyama, Mori et al. 2004), thereby forming a potential alternative to direct cortico-motoneuronal control in cases of corticospinal tract damage. Strengthening of reticulospinal influence on propriospinal and motor neurons has been demonstrated in conjunction with functional improvement following CNS injury (Zaaimi, Edgley et al. 2012, Bachmann, Lindau et al. 2014). Reticulospinal axons may be uniquely suited to mediate anatomical detours relaying cortical input around CNS damage to spinal interneurons due to the fact that they project both excitatory and inhibitory inputs onto propriospinal neurons, in contrast to cortiocospinal, vestibulospinal, and rubrospinal tracts which have been shown to have almost exclusive excitatory inputs (Du Beau, Shakya Shrestha et al. 2012, Brockett, Seenan et al. 2013), as identified based on analysis of neurotransmitter phenotypes. Also with regard to the formation of de novo circuits, the propriospinal cell bodies are in close anatomical approximation to reitculospinal axon terminals in laminae VII and VIII(Reed, Shum-Siu et al. 2006, Brockett, Seenan et al. 2013, Mitchell, McCallum et al. 2016)

Until recently, it was thought that reticulospinal input was required for locomotion, although rythymic leg movements can still be seen in SCl rats after 5-HT antagonist application. Interestingly, un-injured rats showed no gait deficits 
when given the same 5-HT antagonists . A major role for thoracic propriospinal neurons in the indirect transmission of reticulospinal locomotor input was demonstrated nicely in the neonatal rat (Zaporozhets, Cowley et al. 2006, Cowley, Zaporozhets et al. 2010, Zaporozhets, Cowley et al. 2011) and subsequently in the adult rat (Cowley, MacNeil et al. 2015) after contralateral staggered transections abolishing supraspinal input to the lumbosacral cord. 5HT agonist application in the thoracic segments improved the locomotor patterns even further.

With regard to their anatomical location, long descending propriospinal axons travel in the periphery of the anterior and lateral funiculi (see Flynn et. al., 2011), thus constituting part of the 'outer rim' of the spinal cord in which residual axons have been reported in post-mortem studies of humans with clinically classified motor-complete injuries(Kakulas and Kaelan 2015). A greater percentage of long- as opposed to short propriospinal neurons were found to be spared following a low thoracic contusion injury in rats, and perhaps importantly, the number of spared axons did not differ significantly between injury severities (Conta Steencken and Stelzner 2010).

In summary, the reticulospinal-propriospinal system has been shown to play a significant role both in posture/locomotion, and in volitional movement, as an alternative pathway for descending corticospinal input onto spinal motor- and interneurons. Based on these physiological observations, as well as their anatomical proximity, the location of descending axons, and reported sparing 
following experimental injuries of different severities, both pathways deserve increased study as it relates to recovery of motor function after spinal cord injury.

Descending propriospinal pathways have been studied in non-injured humans using electrophysiological methods (Piesiur-Strehlow and Mienck, 1980, Meinck and Piesiur-Strehlow, 1981a, Delwaide and Crenna, 1984b, Kagamihara et al., 2003). These studies reported modulation of leg muscle excitability consistent with evidence suggesting both spinal as well as a supraspinal reflex pathways, differentiated by the latency at which the modulation was observed. One study found that interlimb reflexes were observed during gait, but not during sitting or standing, leading to the hypothesis that the spinal networks involved could be flexibly engaged in a task-specific manner (Dietz, Fouad et al. 2001, Dietz and Michel 2009). Studies examining the role of the arms in recovery of locomotor function after $\mathrm{SCl}$ suggest that arm afferents provide task-specific influence which can improve gait parameters and leg muscle activation patterns after SCl (Visintin and Barbeau, 1994, Tester et al., 2011). 


\section{CHAPTER II}

\section{DEVELOPMENT OF METHODOLOGIES FOR IDENTIFICATION OF DESCENDING INFLUENCE ON LUMBOSACRAL MOTOR EXCITABILITY}

\section{Functional NeuroPhysiological Assessment (FNPA)}

\section{Background}

The most widely utilized assessment tool for the characterization of motor function after human $\mathrm{SCl}$ is International Society of Neurological Classification of Spinal Cord Injury (ISNCSCI) American Spinal Injury Association Impairment Scale (AIS) (Kirshblum, Waring et al. 2011). While this method of classification has been shown to effectively classify $\mathrm{SCl}$ severity into broad categories which correlate with functional ability (Middleton, Truman et al. 1998, Marino and Graves 2004), several limitations exist which limit its utility in SCI research. These include the subjectivity of motor and sensory scores, inability to designate hyper- versus hypoesthesia within the 'impaired' score, the lack of motor assessment for spinal levels T2-L1, and the inability to detect differences and/or changes in neuromuscular control of multi-muscle activation patterns accompanying functional tasks, which may be altered post $\mathrm{SCl}$ (Marino, Barros et al. 2003, Marino and Graves 2004, McKay, Lee et al. 2005, Marino, Jones et 
al. 2008, Kirshblum and Waring 2014). Limitations in the classification of injury using the AIS are evident in the considerable functional heterogeneity that exists within each classification (A-D) of the ISNCSCI (Sherwood, McKay et al. 1996, Behrman, Ardolino et al. 2012, Buehner, Forrest et al. 2012). This issue is of particular concern since AIS classifications are commonly used to stratify participants in clinical intervention studies.

At present there is a need to develop quantitative and sensitive methods for analysis of neuromuscular function below the level of $\mathrm{SCl}$, which possess sufficient sensitivity to 1) identify residual connectivity in descending motor pathways which may underlie the observed voluntary movement in motor complete individuals in the presence of epidural stimulation (Harkema, Gerasimenko et al. 2011, Angeli, Edgerton et al. 2014) 2) aid in stratification of subjects to reduce the considerable heterogeneity present within the $\mathrm{SCl}$ injury spectrum which currently impedes the interpretation of basic science as well as clinical studies in humans, and 3) detect subclinical changes in neurophysiological status as a result of natural recovery or in response to a specific intervention strategy (Ellaway, Kuppuswamy et al. 2011). Additional quantitative, sensitive, and objective techniques for measurement of neuromuscular function post SCI therefore represent a critical need both for clinicians and researchers alike (Steeves2007, Boakye2012).

Objective assessment of $\mathrm{SCl}$ motor function using multi-muscle surface EMG recordings during a standardized set of movement tasks and reflex tests, termed the brain motor control assessment (BMCA), can provide a quantifiable 
alternative to the subjective assessment of muscle strength used in the AIS (Sherwood, McKay et al. 1996). This method is able to detect repeatable and quantifiable increases in EMG activity with volitional effort in leg muscles graded "0" (no visible or palpable contraction) on the AIS (Sherwood, McKay et al. 1996). It was further demonstrated that motor recovery can be quantified in increased EMG amplitudes in prime movers, decrease in co-activation, decrease in time from onset to peak amplitude, as well as normalization of multi-muscle activation patterns (McKay, Lim et al. 2004, McKay, Ovechkin et al. 2011).

In addition to the measurement of muscle activation in response to specific volitional movement attempts, the BMCA also has provided evidence of subclinical descending influence on lower limb muscle activation in subjects with no detectable volitional activation below the injury (Dimitrijevic, Hsu et al. 1992). Three different maneuvers/reflex tests have been used to identify residual descending influence on segmental excitability after $\mathrm{SCl}$, including reinforcement maneuvers, tonic vibratory reflex testing, suppression of the plantar stimulation (Babinski) response. Sherwood et al. (Sherwood, Dimitrijevic et al. 1992) reported a positive finding in at least $1 / 3$ maneuvers in $74 / 88 \mathrm{SCl}$ participants with no detectable volitional motor unit activation, suggesting residual sparing may be present in a majority of individuals classified as motor complete. Reinforcement maneuvers involve the generation of maximal voluntary contraction in muscles above the level of injury. Three maneuvers were standardized: deep breath and hold, neck flexion with resistance, and shoulder shrug. These maneuvers were chosen since the muscles involved are all 
innervated by the high cervical segments and cranial nerves, meaning these muscles would be unaffected by the vast majority of SCls. Positive responses were identified by the appearance of delayed EMG activity in muscles below the level of lesion, usually accompanied by extension of the extremities. Two different types of positive responses were described, termed reinforcement maneuver response 1 and 2, respectively $\left(\mathrm{RMR}_{1}\right.$ and $\left.\mathrm{RMR} 2\right)$ (Dimitrijevic, Dimitrijevic et al. 1984). RMR1 was defined as low amplitude EMG activity observed in 1-3 muscles, with an onset time of $0.8-1.2$ seconds, while $\mathrm{RMR}_{2}$ occurs more commonly, consisting of larger amplitude activity in all muscles tested, with an onset time of 2-3 seconds (Dimitrijevic, Dimitrijevic et al. 1984). It was postulated that these positive responses represent "residual suprasegmental influence...transmitted through widespread plurisegmental excitation of interneurons, resulting in generalized excitation of spinal motor cells" (Dimitrijevic, Dimitrijevic et al. 1984).

The tonic vibratory reflex consists of tonic muscle activation in response to vibration of the same muscle, seen in neurologically intact human subjects (De Gail, Lance et al. 1966). Experiments in the decerebrate cat revealed that the reflex could be abolished by lesion of the lateral vestibulospinal and pontine reticulospinal tracts (Gillies, Burke et al. 1971, Gillies, Burke et al. 1971), and thus is thought to be mediated by supraspinal neuclei. Dimitrijevic and colleagues reported volitional muscle activation was observed during tonic vibration in spinal cord injured subjects who demonstrated no volitional activation ability otherwise (Dimitrijevic, Spencer et al. 1977). In a similar study, the same group reported the 
presence of the reflex in 67 of 88 clinically complete SCl subjects (Sherwood, Dimitrijevic et al. 1992).

Suppression of the Babinski response also has been suggested to identify discomplete SCl. It is identified by a quantifiable reduction of EMG evoked by the response during volitional suppression attempts as compared to the response amplitude from trials in which the subject made no suppression attempt (Cioni, Dimitrijevic et al. 1986). Of the three methods, suppression of the plantar reflex was the most infrequently observed in discomplete subjects (Sherwood, Dimitrijevic et al. 1992), and appears to be the least reliable, as a positive Babinski response is not evoked in all individuals with $\mathrm{SCl}$.

\section{Protocol Development}

In order to objectively quantify voluntary motor capacity and investigate the prevalence and extent of subclinical motor activity in individuals with $\mathrm{SCl}$, the FNPA was developed as a modified and expanded version of the BMCA protocol. The number of muscles from which EMG was recorded was expanded to 16 muscles bilaterally ( 32 total), chosen to represent the maximum possible number of spinal segmental levels (Fig. 1). EMG electrodes were placed midbelly (unless otherwise noted) on the following muscles bilaterally: sternocleidomastoid, upper trapezius, scalene, biceps brachii, triceps brachii, extensor carpi radialis, flexor carpi radialis, flexor digitorum profundus, adductor policis brevis, abductor digiti quinti, external intercostals, rectus abdominus, erector spinae, rectus femoris, adductor magnus, vastus lateralis, tibialis anterior, soleus, extensor digitorum longus, and soleus. Electrode placement was altered 
for rectus abdominus, with placement just distal to ribs and approximately two inches from midline. External Intercostal recording electrodes were placed at the sixth intercostal space along the anterior axillary line. Erector spinae electrodes were placed at the T10 vertebral level, two centimeters lateral from midline. The ASIA exam does not include any motor tests for the thoracic segments, while the BMCA protocol included axial muscles, but did not report analysis of these. Further, the protocol was expanded to include volitional isolated movement attempts for each muscle (Fig. 1). The protocol begins with 5 minutes of relaxation for assessment of baseline EMG activity, followed by reinforcement maneuvers. These consist of deep breath and hold, neck flexion with resistance, and shoulder shrug (Sherwood, Dimitrijevic et al. 1992). In addition, bilateral multi-joint leg flexion and extension attempts also are included to quantify volitional activation in participants who can perform multiple, but not single joint movements. Each maneuver is cued by a 3-second audible tone, with subjects instructed to begin the attempt at the start of the tone, and relax when the tone ended. Reflex tests include plantar stimulation, tests for ankle clonus, patellar and Achilles tendon taps, and patellar and Achilles vibration. The EMG signals are recorded on a 32-channel Eclipse Neurological Workstation (AXON Systems, Inc., Hauppauge, New York, NY, USA) with a sampling rate of $2 \mathrm{kHz}$ per channel and a bandpass of $30 \mathrm{~Hz}$ to $2 \mathrm{kHz}$.

Volitional muscle activation after SCI

In order to assess the sensitivity of the FNPA in the identification and quantification of voluntary muscle activity after $\mathrm{SCl}$, we analyzed EMG from 
volitional movement attempts in the biceps brachii, extensor carpi radialis longus, triceps brachii, tibialis anterior, and soleus muscles to investigate their correlation with AIS motor score and classification in 24 participants (Li, Atkinson et al. 2012). As classed by the AIS, 10 patients were AIS A, 4 were AIS B, 2 were AIS C, and 8 were AIS D (Table 1). AIS examination was performed within 48 hours of the FNPA in each participant.

To quantify responses, data are rectified and mean and root-mean-square (RMS) values were calculated for each attempt per muscle. Averages and standard deviations of mean and RMS values per muscle were calculated from three attempts in all events except relaxation. Relaxation was divided into 30 second intervals. Average and standard deviation of mean and RMS values were determined from 10 intervals in 5 minute of relaxation per muscle per person. Data was down sampled to $20 \mathrm{~Hz}$ for $\mathrm{EMG}$ envelope.

In 4 patients, EMG was recorded in muscles with an AIS motor score of zero (Fig. 2). In addition, trunk muscle activity was directly assessed in SCI participants, with EMG activity detected in 19/24 participants (cf. Fig. 2). Due to scarcity of AIS motor scores 1-3 in our participant population (Table 2), group comparisons of AIS motor score and RMS EMG activity was limited to AIS motor scores of 0 and 5 , except for the triceps brachii muscle, for which the AIS motor score of 4 was also included. Group RMS values by muscle are provided in Table 3 and visually presented in Figure 3 . RMS values and AIS motor scores were found to be well correlated overall, and in 4 of 5 individual muscles (Table 4). Difference in RMS values between muscles given an AIS motor score of 5 
and those given an AIS motor score of 0 was statistically significant for the triceps brachii, tibialis anterior, and soleus muscles $(p \leq .02)$, and approached significance for the biceps brachii and extensor carpi radialis muscles $(P=.059$ and .052 respectively). Comparison of the RMS values with AIS motor scores or 4 or 0 was made in the triceps brachii muscle, and these were also found to be statistically different $(p=.008)$. Additionally, sensitivity to changes in muscle activation over time as quantified by EMG RMS values can be observed (Fig. 4). In this representative example, an increase in EMG amplitude is noted for the biceps muscle between the first and second assessment, both of which occurred while the AIS motor score was 0.

The results of this study provided evidence that this approach can improve the resolution of residual motor capacity after $\mathrm{SCl}$. The detection of time-locked EMG activity during repeated movement attempts in muscles given an AIS motor score of 0 underscores this point. In fact, we identified such activity in two AIS A participants, one of whom converted to an AIS B during the course of the study. Whether neurophysiological examination can reliably predict the recovery of motor function based on apparent subclinical EMG activity deserves further study.

The inclusion of axial muscles innervated by the thoracic segments in this study is also noteworthy. Currently the motor score distribution among AIS injury levels between T2-L1 allows no distinction across this broad range of injury levels, since the motor score is $50 / 100$ for all such individuals (Graves, Frankiewicz et al. 2006). In addition to the advantage of allowing objective and 
quantifiable characterization of motor capacity after $\mathrm{SCl}$, the multi-muscle recordings may be further analyzed to identify both quantitative and qualitative differences in the spatiotemporal coordination of spinal motor pools from multiple bilateral segmental levels (Lee, Lim et al. 2004, Lim, Lee et al. 2005, McKay, Lee et al. 2005).

In summary, neurophysiological assessment of motor function after $\mathrm{SCl}$ via multi-muscle surface EMG can provide a quantitative and sensitive alternative for the characterization of motor function after human SCl. This method allows detection of subclinical descending influence on motor activity, and importantly can be used to objectively monitor change in function across time.

\section{Development of neurophysiological pathways assessment}

\section{Background}

While the FNPA can provide quantitative, sensitive assessment of volitional motor capacity as well as identify subclinical sparing of descending influence on spinal excitability, it is not designed to identify the specific descending pathways/ neuronal populations involved. Electrophysiological methods for investigating descending pathways exist, but typically are studied in the presence of volitional or postural muscle activity, making them ill-suited for the current purpose. Alternatively, some have studied descending influence using a condition-test paradigm in which the effect of conditioning stimuli on spinal motor excitability is determined by changes in the amplitude of the H-reflex. . The $\mathrm{H}$ reflex is elicited via electrical stimulation of peripheral nerves to induce excitation of peripheral afferents (group I in particular) which mediate segmental 
reflexes via mono- and oligosynaptic connections to motor neurons serving the muscles from which the stimulated afferents project. nerve undergoing stimulation (Magladery and Mc 1950, Magladery, Porter et al. 1951, PierrotDeseilligny 2005). A major limitation to this technique, however, is that the $\mathrm{H}$ reflex is not reliably evoked in many muscles, making the soleus muscle the most widely used for such studies (Zehr 2002). Since the soleus receives its innervation from the S1 and S2 segments, information can only be obtained related to changes within a single unilateral motor pool representing only the most distal segments of the lumbosacral enlargement (Sharrard 1964, Kendall, McCreary et al. 1983).

In order to gain more information as to the nature and extent of the descending excitation and/or inhibition on the lumbosacral motor pools, multisegmental muscle responses (MMRs) evoked by transcutaneous spinal stimulation were utilized, allowing us to monitor excitability changes in motor pools throughout the lumbosacral enlargement, as represented by multiple, bilateral, proximal and distal leg muscles (Courtine, Harkema et al. 2007, Minassian, Persy et al. 2007). The use of an evoked response precludes the need for volitional activation, and the finding that individuals with chronic, motor complete $\mathrm{SCl}$ regain the ability to activate muscles volitionally in the presence of epidural stimulation (Harkema, Gerasimenko et al. 2011, Angeli, Edgerton et al. 2014) suggests that electrical excitation of the lumbosacral circuitry may be required for the observation of residual descending influence after chronic, motor 
complete $\mathrm{SCl}$, as defined by the International Standards for Neurological Classification of Spinal Cord Injury (ISNCSCI) (Kirshblum, Waring et al. 2011).

Single pulses of transcutaneous spinal stimulation delivered posteriorly over the lumbosacral spinal cord evoke MMRs in bilateral proximal and distal lower extremity muscles, via excitation of dorsal root afferents (Minassian, Persy et al. 2007, Ladenbauer, Minassian et al. 2010). Evidence for the reflex nature of MMRs has been reported: responses are inhibited by prior pulses indicating postactviation depression, as well as by vibration of the muscle tendon, and active antagonist muscle contraction (Courtine, Harkema et al. 2007, Minassian, Persy et al. 2007, Dy, Gerasimenko et al. 2010). Similarly, MMRs exhibit task- and phase-dependent modulation during standing and stepping (Courtine, Harkema et al. 2007, Hofstoetter, Minassian et al. 2008, Dy, Gerasimenko et al. 2010). MMRs address some of the limitations inherent to H-reflex modulation studies, making it well suited for reflex testing. Posterior roots are preferentially stimulated, so no M-wave is generated and no antidromic volley exists which would collide with the orthodromic reflex response. The site of stimulation for the MMR overcomes some challenges associated with access to peripheral nerves with H-reflex stimulation (Zehr 2002). Comparison of the H-reflex and MMR during standing and ambulation in both healthy and spinal cord injured subjects illustrates an advantage to the use of MMRs when probing the spinal motor circuitry: this method allows for simultaneous detection of excitability modulation at multiple segments, which can provide information about the level of network 
integration and organization in response to specific sensory stimuli (Courtine, Harkema et al. 2007, Hofstoetter, Minassian et al. 2008, Lavrov, Dy et al. 2008). However, only a few studies have reported use of MMRs thus far, and these have varied in methodology, leading to incongruent reports regarding the nature of the responses following stimulation at a particular rostro-caudal location (Minassian, Persy et al. 2007, Troni, Di Sapio et al. 2011, Roy, Gibson et al. 2012). Further, the extent to which MMRs might be optimized for use in studies examining modulation in response to specific conditioning stimuli has not been previously addressed. As discussed, MMR modulation has been demonstrated to be analogous to that of the peripherally evoked $\mathrm{H}$-reflex. However, to date none of these studies has undertaken analysis methods which account for inherent differences among motor pools, limiting and otherwise complicating interpretation of this data. Such analyses are needed for a valid comparison of the effects among individual muscles. We hypothesized that the characteristics of MMR recruitment curves obtained from different leg muscles will reflect a relative preferential activation of proximal or distal motor pools based on their rostrocaudal arrangement along the lumbosacral enlargement (Fig. 5).

Characterization of MMR activation patterns evoked by transcutaneous spinal cord stimulation

We first sought to characterize the selectivity of recruitment of lumbosacral motor pools in uninjured individuals using transcutaneous spinal stimulation along the rostro-caudal axis of the lumbosacral enlargement. What follows is a synopsis of the results, which support the current studies, and have been 
published elsewhere (Sayenko, Atkinson et al. 2015). We obtained MMR recruitment curves from bilateral $\mathrm{RF}, \mathrm{VL}, \mathrm{MH}, \mathrm{TA}, \mathrm{MG}$, and $\mathrm{SOL}$ via surface electrodes during stimulation at multiple rostro-caudal levels in 15 neurologically intact participants. This was accomplished by placing the stimulating electrode at the T10-T11, T11-T12, T12-L1, and L1-L2 interspinous spaces and, using $2 \mathrm{~mA}$ increments, increasing the stimulation intensity from 2 to $100 \mathrm{~mA}$, or the maximum tolerable intensity. At each stimulation intensity, a minimum of 3 stimuli were delivered.

The results demonstrated that the relative order of activation (i.e. the relative increase or decrease in MMR threshold for a particular muscle) varied predictably based on location of stimulation (fig. 6). Specifically, the thresholds of knee muscles, whose motor pools are located more proximally within the lumbosacral segments, were lower than distal muscles' thresholds when stimulation was delivered to more proximal segments (i.e. T10-T11, T11-T12) while the reverse was true for more distal sites of stimulation. In addition to the selective recruitment of proximal or distal leg muscles at threshold intensities based on the site of stimulation, MMR thresholds in all muscles increased as the site of stimulation was moved proximally. Further, and in agreement with previous studies (Minassian, Persy et al. 2007, Troni, Di Sapio et al. 2011, Roy, Gibson et al. 2012), the rostro-caudal level of stimulation also appeared to affect the preferential activation of afferent structures, with the potential of activation of motor fibers noted at more caudal stimulation locations (Fig. 7). 
The location of stimulation also influenced the rate of recruitment as measured by the slope of the recruitment curve (Fig. 8). Posterior leg muscles, $\mathrm{MH}, \mathrm{MG}$, and SOL, consistently demonstrated greater rates of recruitment, which agrees with known inter-muscle differences in sensitivity to afferent stimulation (Edgerton, Smith et al. 1975, Meunier, Pierrot-Deseilligny et al. 1994, PierrotDeseilligny 2005) likely to reflect intrinsic differences among motor pools relative to functional roles.

Lastly, left-right asymmetry was noted (Fig. 9). In experimental sessions, we attempted to minimize asymmetry by medial-lateral movement of the stimulating electrode, however this had little to no effect on the observed phenomena. This result appears to suggest the asymmetries noted are the result of anatomical and/or physiological differences in individual muscles, although interestingly the left-right MMR asymmetries within an individual did not appear to be consistently related to handedness or a dominant functional side.

The results of this study demonstrated that MMRs could be evoked in bilateral knee and ankle muscles, with predictable variance in threshold and recruitment characteristics among muscles studied. It was shown that through optimization of the location of stimulation, it is possible to evoke submaximal MMRs in all muscles simultaneously, which is ideal for conditioning studies (Crone, Hultborn et al. 1990). In particular, due to the relatively slower rate of recruitment of proximal muscles $\mathrm{VL}$ and $\mathrm{RF}$, and the potential for direct motor contamination at more caudal levels or stimulation, a more proximal stimulation location appears preferable. While previous studies of MMR modulation have 
identified a stimulation location based on bony landmarks, the present data suggest that operationally, the location of stimulation may be better standardized across individuals when identified based on relative MMR recruitment thresholds. With regard to the use of MMRs in a condition-test paradigm, these implications are discussed further in the next chapter. 


\section{Table 1}

Demographic and clinical characteristics in 28 patients with $\mathrm{SCl}^{*}$

\begin{tabular}{ccccc}
\hline Characteristic & $\begin{array}{c}\text { Full Sample (\# } \\
\text { assessments) }\end{array}$ & $\begin{array}{l}\text { Motor complete } \\
\text { (AIS A \& B) }\end{array}$ & $\begin{array}{c}\text { Motor Incomplete } \\
\text { (AIS C \& D) }\end{array}$ & $\begin{array}{l}\text { Neurologically } \\
\text { Intact }\end{array}$ \\
\hline Sex: & $24(58)$ & 13 & 7 & 3 \\
Women & $4(8)$ & 1 & 3 & 1 \\
Age: (y) & $38 \pm 15$ & & & \\
Injury Level & 17 & 8 & 9 & NA \\
Cervical & 7 & 6 & 1 & NA \\
Thoracic & & & & \\
Time Since SCl & & & & \\
(years) \\
$\leq 1$
\end{tabular}

NOTE: There were 28 total volunteers, who participated in 66 total assessments. Of the $62 \mathrm{SCl}$ assessments, 32 have an associated ASIA exam (within 3 days).

(Li et al., 2012) 


\section{Table 2}

Distribution of AIS motor scores at spinal cord levels corresponding to muscles of interest.

\begin{tabular}{|c|c|cccccc|}
\hline $\begin{array}{c}\text { Associated } \\
\text { Muscle }\end{array}$ & Level & $\mathbf{0}$ & $\mathbf{1}$ & $\mathbf{2}$ & $\mathbf{3}$ & $\mathbf{4}$ & $\mathbf{5}$ \\
\hline biceps & $\mathrm{C} 5$ & 5 & 1 & 0 & 2 & 3 & 34 \\
ECR & $\mathrm{C} 6$ & 6 & 1 & 0 & 2 & 3 & 33 \\
triceps & $\mathrm{C} 7$ & 7 & 4 & 1 & 1 & 11 & 26 \\
TA & $\mathrm{L} 4$ & 35 & 0 & 2 & 1 & 7 & 17 \\
Soleus & $\mathrm{S} 1$ & 33 & 2 & 1 & 1 & 4 & 20 \\
\hline Total & & 86 & 8 & 4 & 7 & 28 & 130 \\
\hline
\end{tabular}

(Li et al., 2012) 


\section{Table 3}

Summary statistics of RMS EMG activity at each muscle of interest by ISNCSCI motor score*.

\begin{tabular}{|l|c|cccccc|}
\hline Muscle & Level & $\mathbf{0}$ & $\mathbf{1}$ & $\mathbf{2}$ & $\mathbf{3}$ & $\mathbf{4}$ & $\mathbf{5}$ \\
\hline Bicep & C5 & $\begin{array}{c}1.7 \\
{[1.7,1.7]}\end{array}$ & 5.4 & & $\begin{array}{c}52.1 \\
{[48.5,55.7]}\end{array}$ & $\begin{array}{c}22.2 \\
{[19.7,36.1]}\end{array}$ & $\begin{array}{c}75.3 \\
{[32.2,131.9]}\end{array}$ \\
\hline ECR & C6 & $\begin{array}{c}5.8 \\
{[4.2,6.5]}\end{array}$ & 29.2 & & $\begin{array}{c}36.3 \\
{[28.6,44.1]}\end{array}$ & $\begin{array}{c}34.2 \\
{[24.4,40.8]}\end{array}$ & $\begin{array}{c}145.1 \\
{[82.1,296.9]}\end{array}$ \\
\hline Tricep & C7 & $\begin{array}{c}1.7 \\
{[1.4,5.0]}\end{array}$ & $\begin{array}{c}5.2 \\
{[2.6,27.5]}\end{array}$ & 0.7 & 17.2 & $\begin{array}{c}13.5 \\
{[5.8,16.2]}\end{array}$ & $\begin{array}{c}43.9 \\
{[13.2,115.8]}\end{array}$ \\
\hline TA & L4 & $\begin{array}{c}0.9 \\
{[0.6,1.6]}\end{array}$ & & $\begin{array}{c}9 \\
{[4.8,13.2]}\end{array}$ & 29.2 & $\begin{array}{c}62.7 \\
{[31.6,114.5]}\end{array}$ & $\begin{array}{c}109.1 \\
{[70.4,193]}\end{array}$ \\
\hline Soleus & S1 & $\begin{array}{c}0.8 \\
{[0.6,1.4]}\end{array}$ & $\begin{array}{c}1.1 \\
{[1.0,1.2]}\end{array}$ & 26.8 & 39.6 & $\begin{array}{c}44.8 \\
{[26.2,63.4]}\end{array}$ & {$[22.7,89.6]$} \\
\hline
\end{tabular}

*at the spinal cord level corresponding to the given muscle. Values are median [interquartile extrema]. Empty cells indicate AIS motor scores not observed in our sample. Cells with single numbers indicate ISNCSCI motor scores observed only once. 


\section{Table 4}

Results of correlation test (nonparametric Kendall correlation test) and ISNCSCI motor score group EMG comparison (nonparametric Kruskal-Wallis test)

\begin{tabular}{|c|c|c|c|}
\hline Muscle & Level & $\begin{array}{c}\text { Correlation }(95 \% \\
\text { CI) }\end{array}$ & $\begin{array}{c}\text { P-value } \\
\text { (ISNCSCI=0 vs. } \\
\text { ISNCSCI=5) }\end{array}$ \\
\hline Bicep & C5 & $0.25[-0.11,0.60]$ & 0.059 \\
\hline ECR & C6 & $0.80[0.42,1.00]$ & 0.052 \\
\hline Tricep & C7 & $0.52[0.34,0.70]$ & 0.02 \\
\hline TA & L4 & $0.53[0.33,0.73]$ & $<0.01$ \\
\hline Soleus & S1 & $0.51[0.28,0.74]$ & $<0.01$ \\
\hline Overall & & $0.85(0.76,0.95)$ & $<0.01$ \\
\hline
\end{tabular}

(Li et al., 2012) 
Figure 1. Diagram and chart showing FNPA muscles and maneuvers.

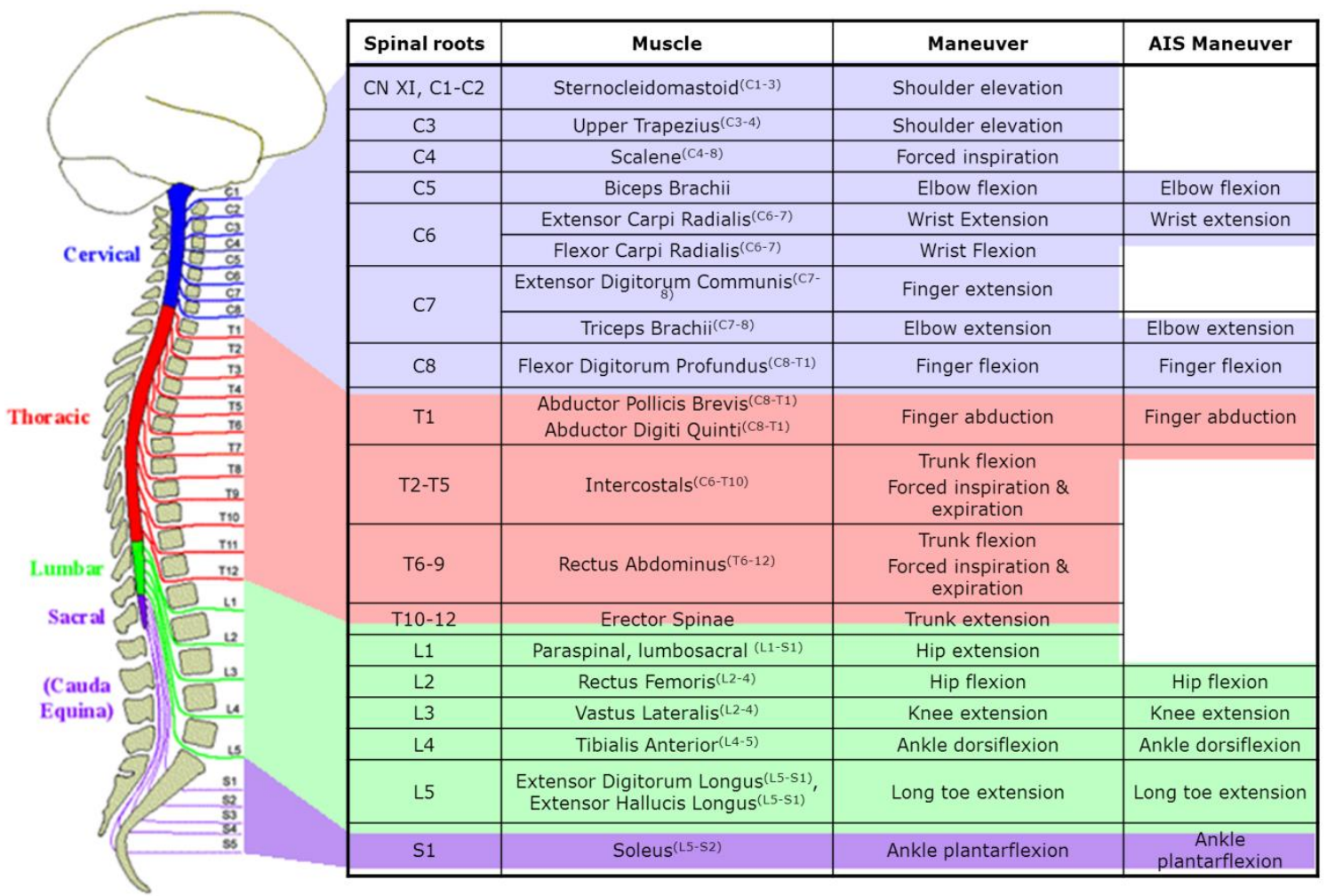

Muscles assessed using both the FNPA and the ISNCSCI examination are listed as well as the maneuvers used for testing and the spinal root level that each muscle represents. AIS = ASIA Impairment Scale. 


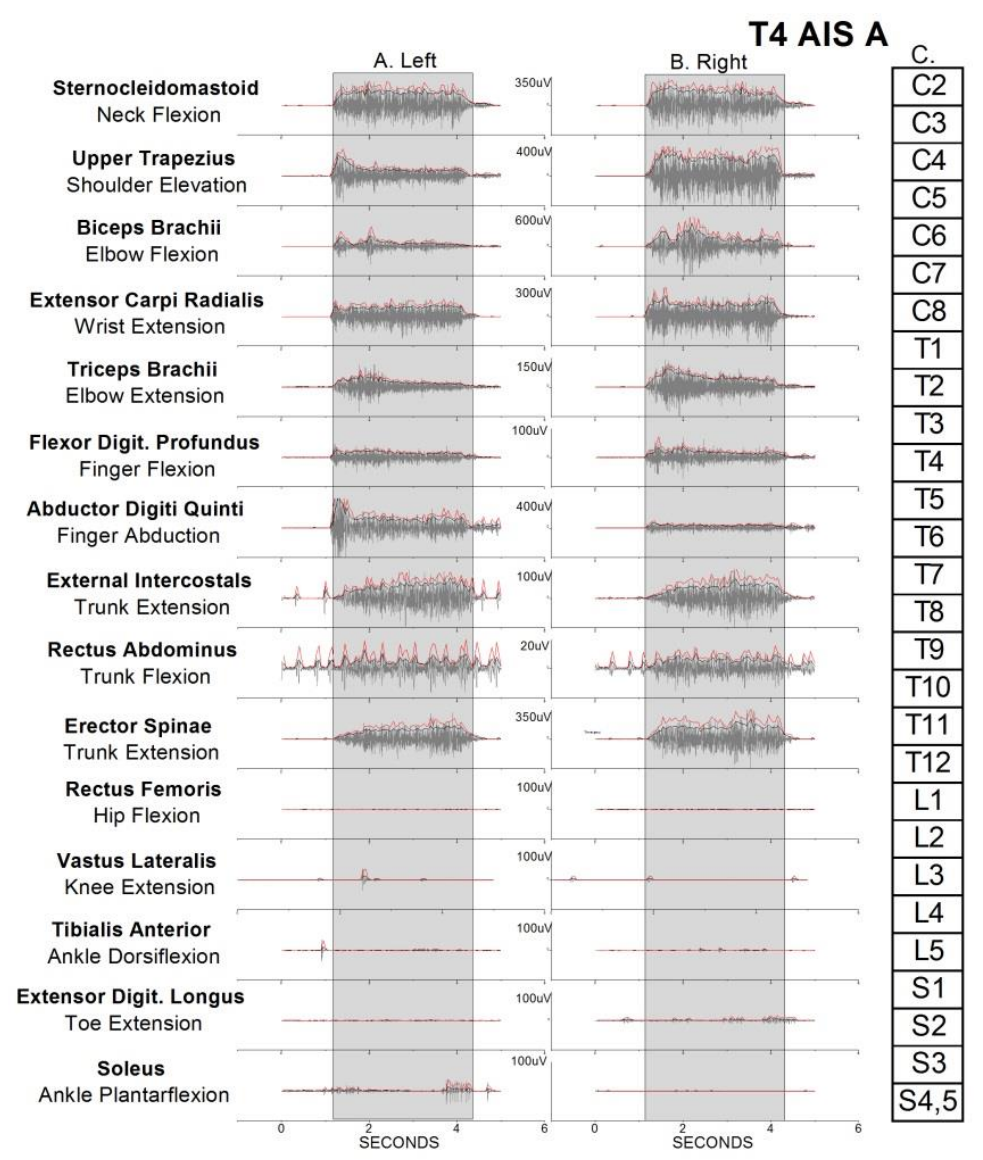

\begin{tabular}{|c|c|c|c|c|c|}
\hline \multicolumn{2}{|c|}{ D. Motor } & \multicolumn{2}{|c|}{ E. Light Touch } & \multicolumn{2}{|c|}{ F. Pin Prick } \\
\hline & & 2 & 2 & 2 & 2 \\
\hline & & 2 & 2 & 2 & 2 \\
\hline & & 2 & 2 & 2 & 2 \\
\hline 5 & 5 & 2 & 2 & 2 & 2 \\
\hline 5 & 5 & 2 & 2 & 2 & 2 \\
\hline 5 & 5 & 2 & 2 & 2 & 2 \\
\hline 5 & 5 & 2 & 2 & 2 & 2 \\
\hline 5 & 5 & 2 & 2 & 2 & 2 \\
\hline & & 2 & 2 & 2 & 2 \\
\hline & & 2 & 2 & 2 & 2 \\
\hline & & 2 & 2 & 2 & 2 \\
\hline & & 0 & 0 & 0 & 0 \\
\hline & & 0 & 0 & 0 & 0 \\
\hline & & 0 & 0 & 0 & 0 \\
\hline & & 0 & 0 & 0 & 0 \\
\hline & & 0 & 0 & 0 & 0 \\
\hline & & 0 & 0 & 0 & 0 \\
\hline & & 0 & 0 & 0 & 0 \\
\hline & & 0 & 0 & 0 & 0 \\
\hline & & 0 & 0 & 0 & 0 \\
\hline 0 & 0 & 0 & 0 & 0 & 0 \\
\hline 0 & 0 & 0 & 0 & 0 & 0 \\
\hline 0 & 0 & 0 & 0 & 0 & 0 \\
\hline 0 & 0 & 0 & 0 & 0 & 0 \\
\hline 0 & 0 & 0 & 0 & 0 & 0 \\
\hline & & 0 & 0 & 0 & 0 \\
\hline & & 0 & 0 & 0 & 0 \\
\hline & & 0 & 0 & 0 & 0 \\
\hline
\end{tabular}

Figure 2. FNPA voluntary movement results in a patient classified as T-4 ASIA.

The EMG activity shown (gray lines) represents the averaged EMG response

from 3 trials over time for primary muscles responsible for each corresponding voluntary movement from the left (A) and right (B) sides. Each EMG response was down-sampled to $20 \mathrm{~Hz}$ and rectified. The mean \pm SD of 3 responses over time was superimposed on the figure (black and red lines, respectively).

Corresponding muscle spinal levels (C), ISNCSCI motor scores (D), light touch $(E)$, and pinprick (F). The shaded gray area indicates the time of command for the specific voluntary movement. This patient clearly shows repeatable, voluntary muscle activity below the lowest intact motor and sensory level identified on the ISNCSCI examination. Digit. $=$ digitorum .

(Li et al., 2012) 
Figure 3. Boxplots of RMS EMG activity for muscles of interest by ASIA Impairment Scale motor score.

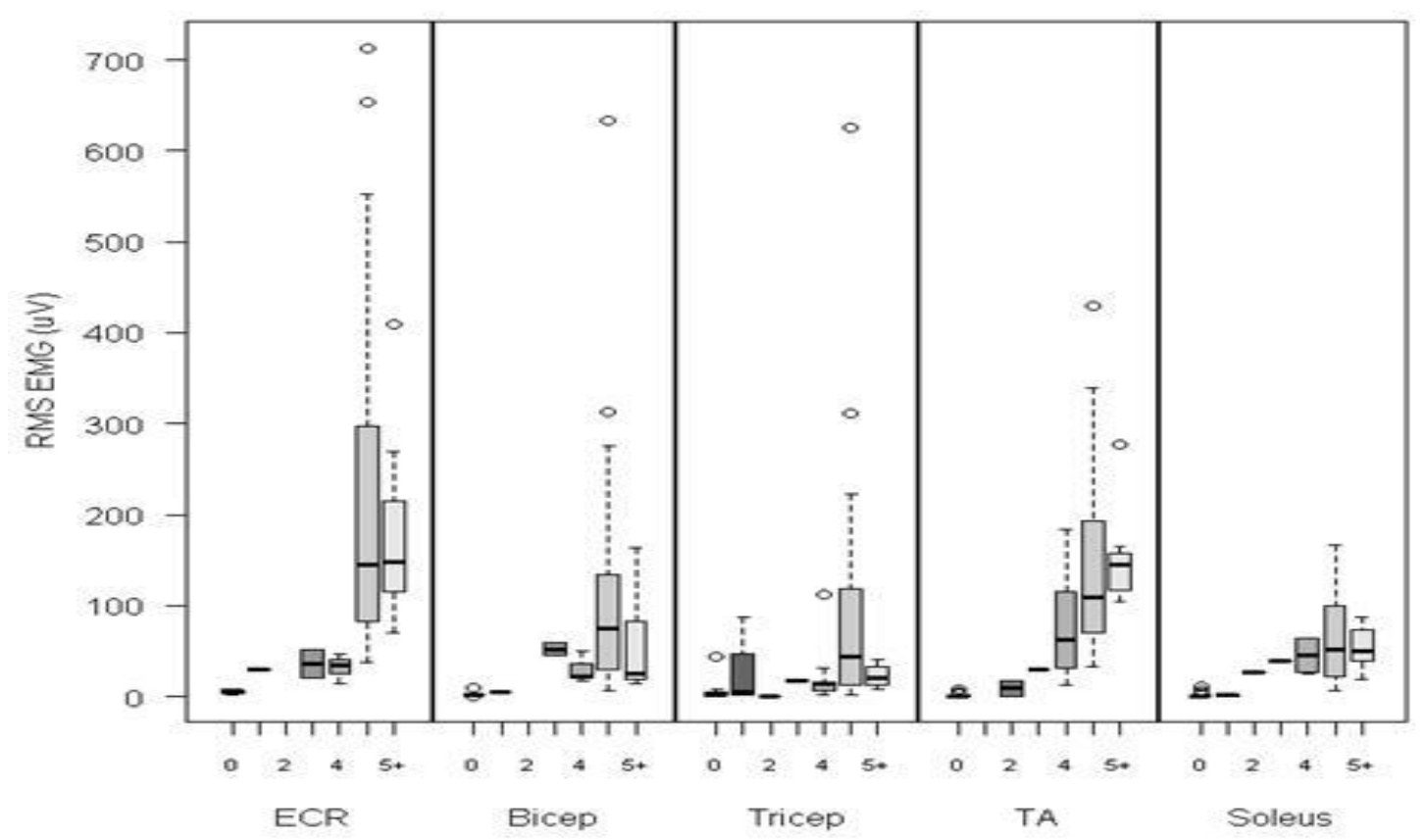

Motor score 5+ indicates noninjured individuals. The lines bisecting the boxes are the medians; the edges of the boxes are the interquartile range (25th and 75 th percentiles); the edges of the whiskers represent the outlier-excluded range (minimum and maximum, excluding outliers); the circles represent outliers, which are points that lie beyond $1.5^{*}$ the interquartile range from the edge of the box.

(Li et al., 2012) 
Figure 4. Recovery of EMG in elbow flexor muscle after SCl.
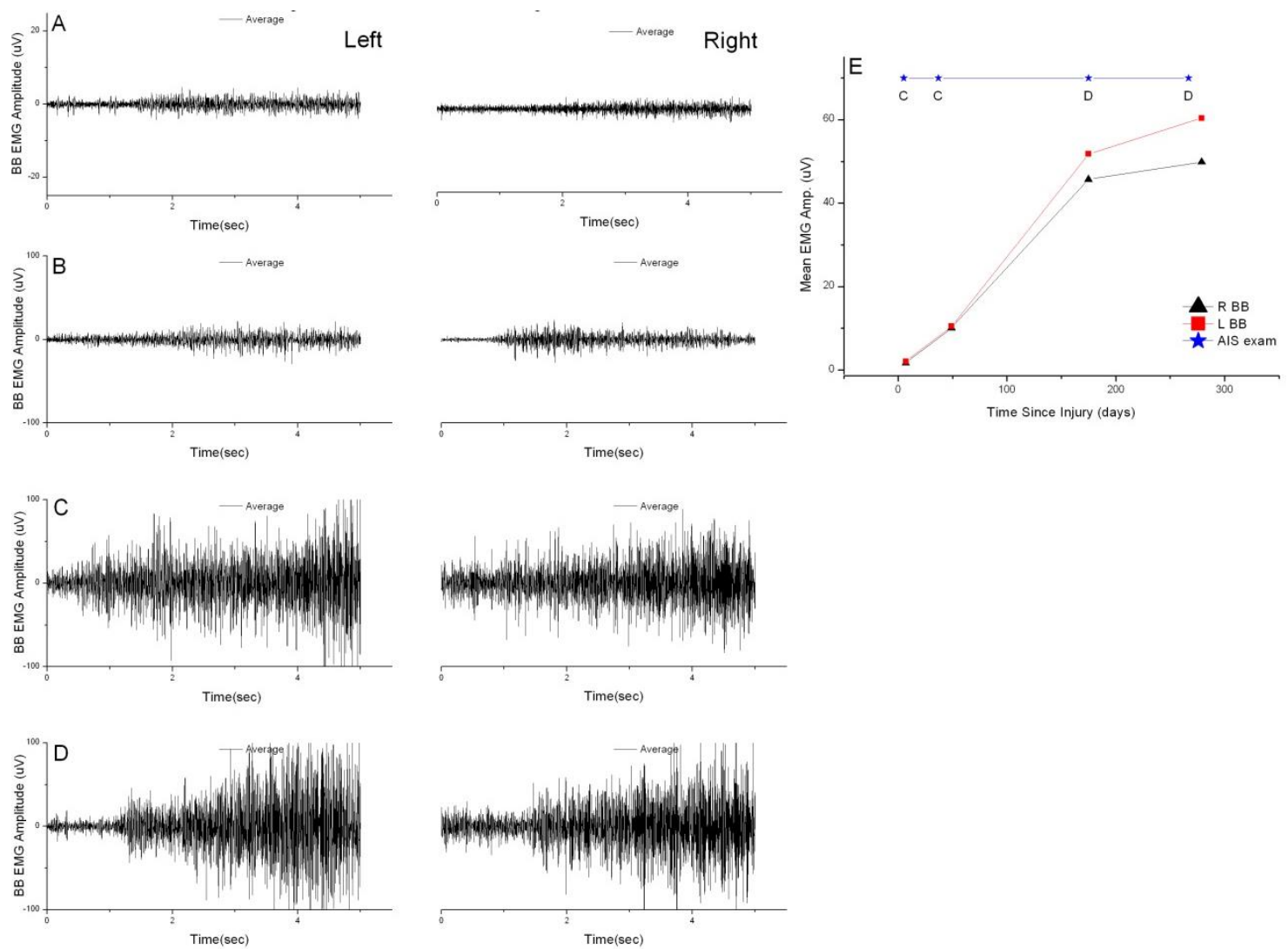

The EMG amplitude (in $\mathrm{mV}$ ) for the averaged response from three 3-second trials recorded from the left $(L)$ and right $(R)$ biceps brachii (BB) muscles at 7 days (A), 49 days (B), 175 days (C), and 279 days (D) post-SCl. Change in RMS values for right and left biceps brachii from the same time points is shown in panel $\mathbf{E}$. Also shown are the dates of the ISNCSCI examinations for this patient $(5,37$, 175, and 267 days postinjury) as well as the ASIA Impairment Scale (AIS) classification for that examination.

(Li et al., 2012) 
Figure 5. Approximate location of individual muscles' spinal motor pools within the lumbosacral enlargement.

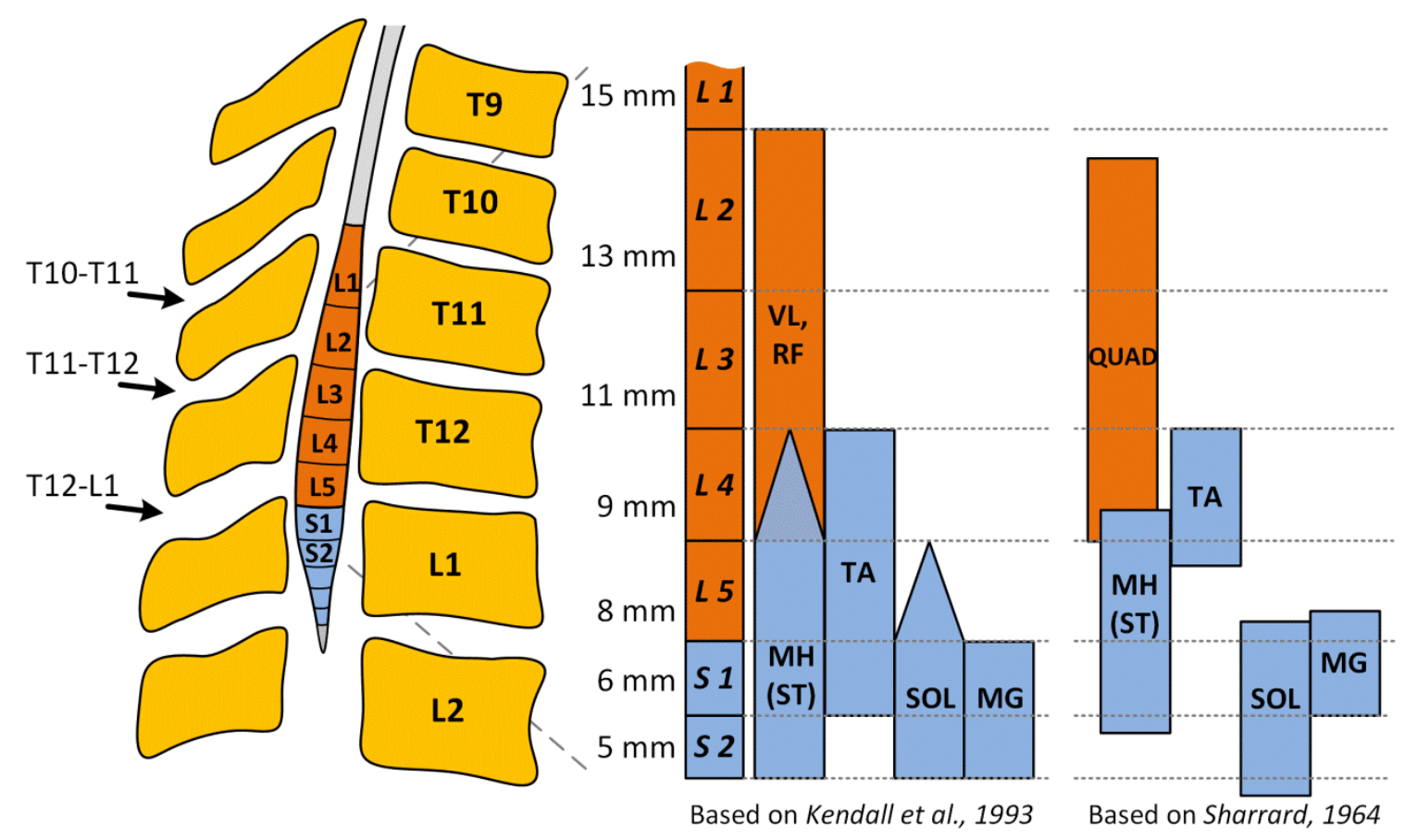

Reconstruction showing the approximate location of transcutaneous electrical spinal stimulation over the lumbosacral enlargement, and the location of the motor pools based on the segmental charts provided by Kendall et al. (27) and Sharrard (52). Figure created using data from Refs. 27 and 52. Triangle endings in Kendall's chart denote agreement of three to four sources out of six from the anatomical and clinical data, whereas square ending bars denote motor pools' localization agreed on in five or six sources. The numbers shown left at each spinal segment are the average length in millimeters of the segment (52). VL, vastus lateralis; RF, rectus femoris; $\mathrm{MH}$, medial hamstring; $\mathrm{ST}$, semitendinosus; TA, tibialis anterior; SOL, soleus; MG, medial gastrocnemius; QUAD, quadriceps. 
Figure. 6. MMRs in one participant during transcutaneous electrical spinal stimulation delivered at multiple lumbosacral levels.

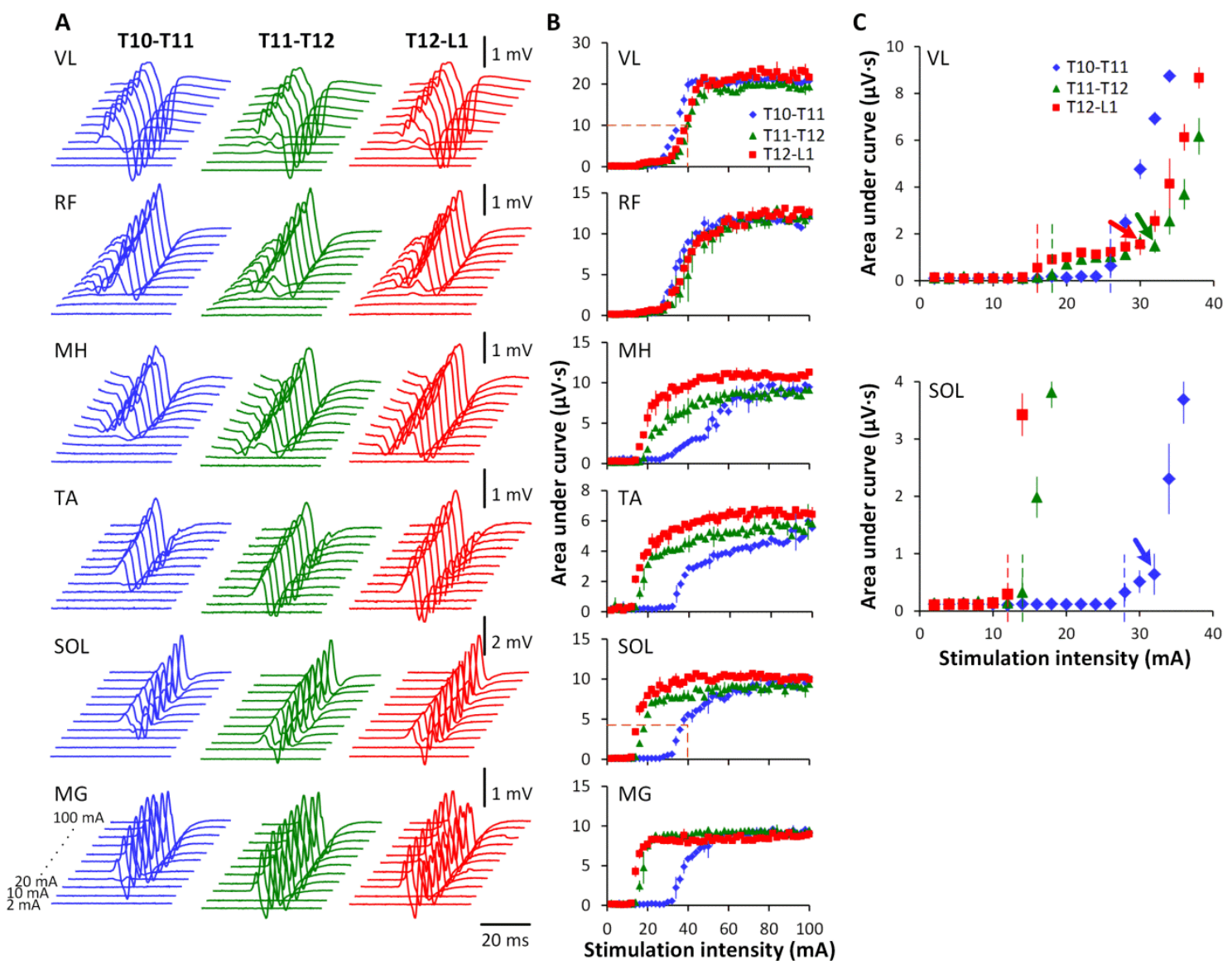

$A$ : the average of three nonrectified MMRs in right muscles at each stimulation intensity from 2 to $100 \mathrm{~mA}$. Shown is the time window between 10 and $55 \mathrm{~ms}$ following the stimulus. $B$ : recruitment curves of right muscles at each location of spinal stimulation. Orange dotted lines on VL and SOL indicate the initial rise of the recruitment curves. $C$ : enlarged ascending fragments of the recruitment curves of $\mathrm{VL}$ and $\mathrm{SOL}$ designated in $B$. Dotted lines of different colors show the muscle activation threshold corresponding to each spinal level of stimulation. 
Arrows indicate the inflection points in the ascending fragments recruitment curves. 
Figure 7. MMRs in one participant during transcutaneous electrical spinal stimulation delivered at different intensities at three spinal levels.
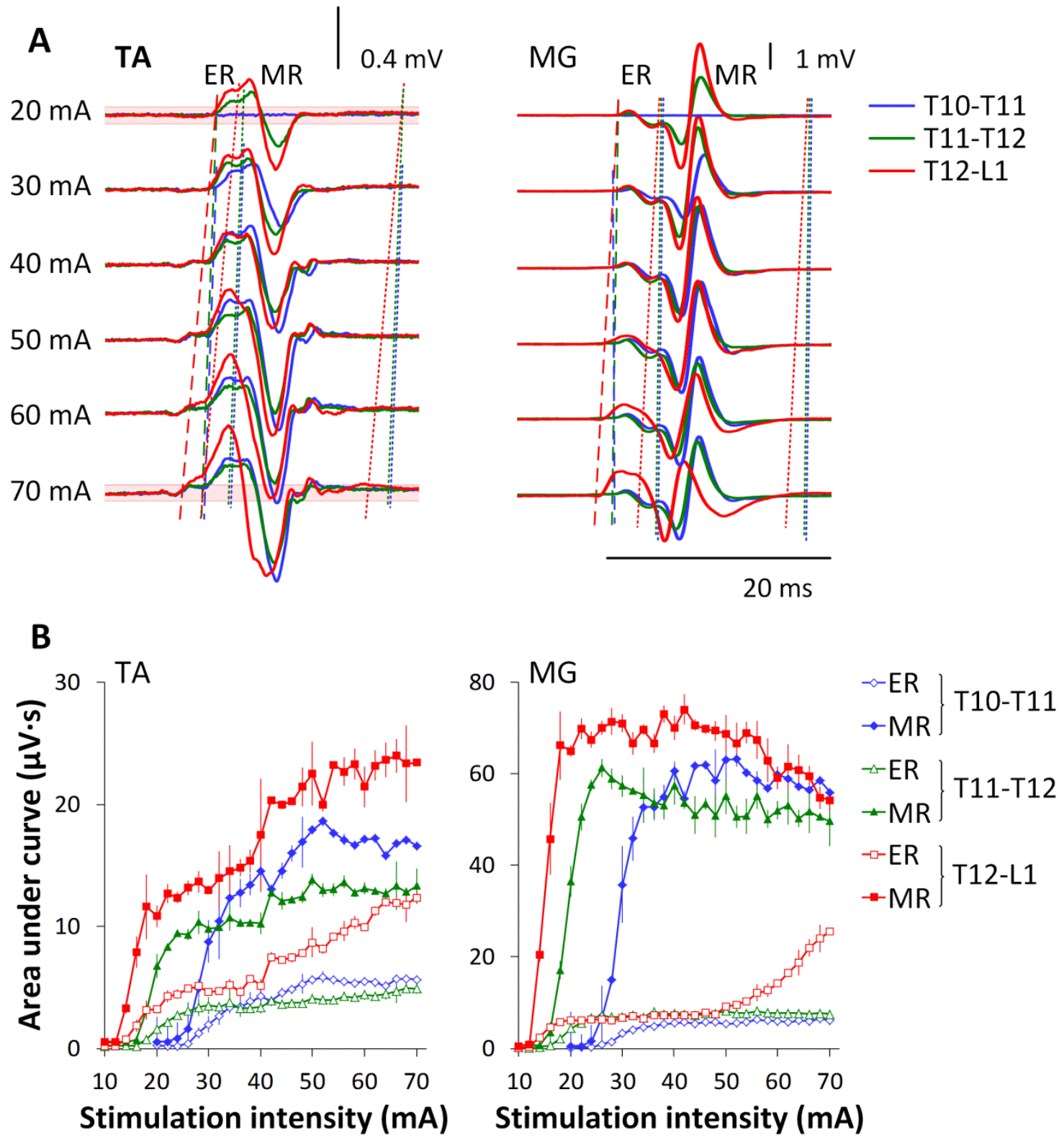

$A$ : the average of three nonrectified responses is shown for each stimulation intensity. Dashed lines of different colors show the onset of the early responses (ER) determined from the ensemble-averaged waveforms and corresponding to each spinal level of stimulation. Note the changes of the onset with increment of the stimulation intensity. Dotted lines indicate the epochs of medium responses 
(MR). Note well-distinguished separation between ER and MR at lower stimulation intensities, and a masked,"smoothed" division between them at higher intensities. Red shadowed areas near the baseline on the top and bottom traces on the left indicate the average magnitude plus 3 SDs before the stimulus during stimulation at T12-L1. B: area of the ERs and MRs during stimulation delivered at each stimulation location.

(Sayenko et al., 2015) 
Figure 8. MMR rate of recruitment as quantified by slope of recruitment curves. Pooled data of the maximal tangential slope of the ascending portions of recruitment curves of right leg muscles at different locations of stimulation.

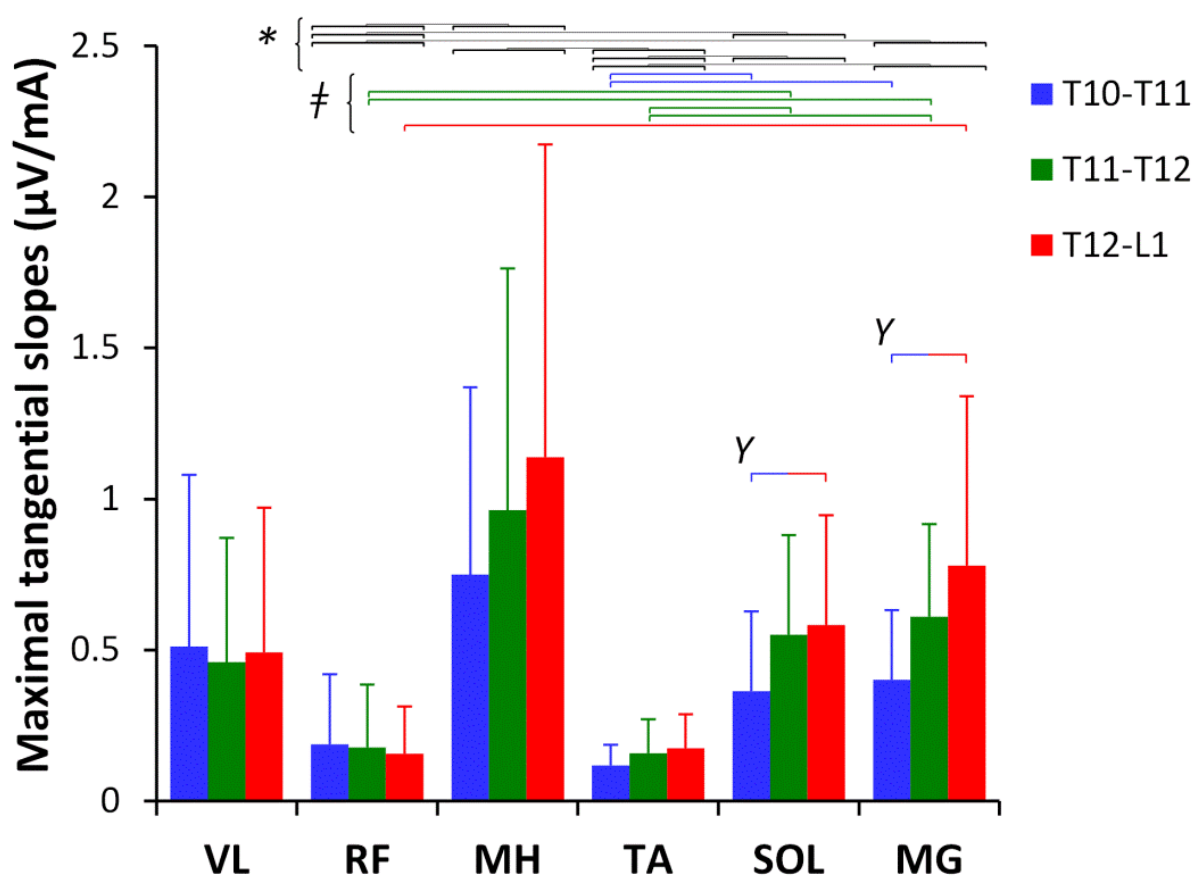

*Black horizontal bars indicate statistically significant differences of the slope between the muscles: RF vs. MH, SOL, MG; MH vs. TA; and TA vs. SOL, MG. F/Blue, green, and red horizontal bars indicate statistically significant differences of the slope between different muscles at T10-T11 (i.e., TA vs. SOL, MH), T11T12 (i.e., RF vs. SOL, MG, as well as TA vs. SOL, MG), and T12-L1 (i.e., RF vs. $M G)$, respectively. Y|Blue-red bars indicate the differences of the slope between T10- T11 and T12-L1 in SOL and MG $(P \leq 0.05)$. 
Figure 9. Left-Right asymmetry of MMRs.

\section{A}
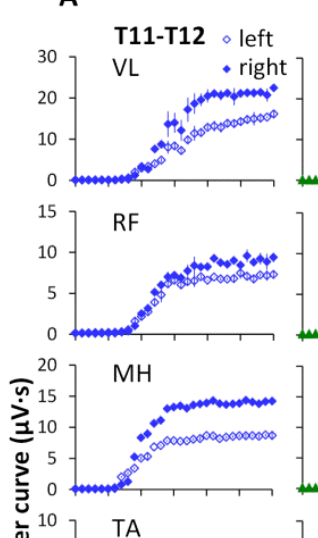

.
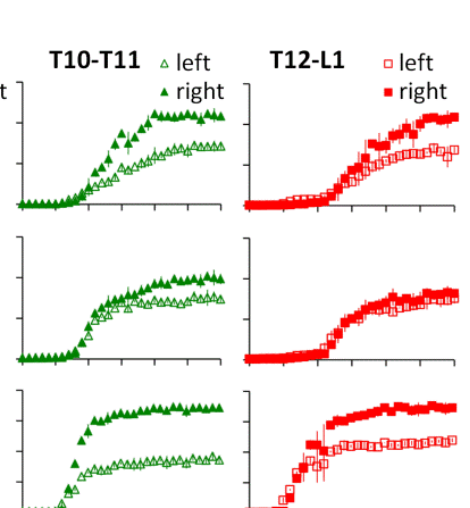

TA

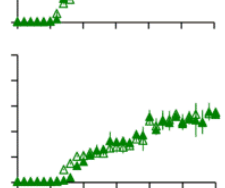

SOL

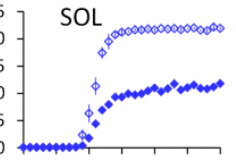

I $\mathrm{MG}$

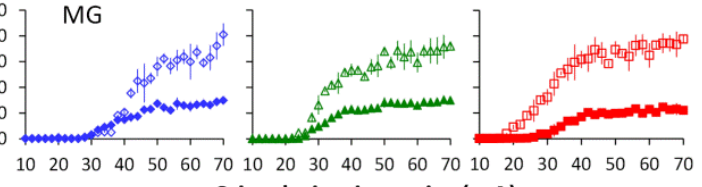
Stimulation intensity ( $\mathrm{mA}$ )

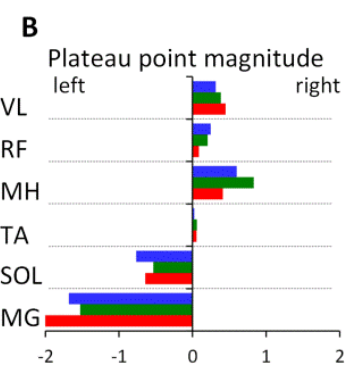

C

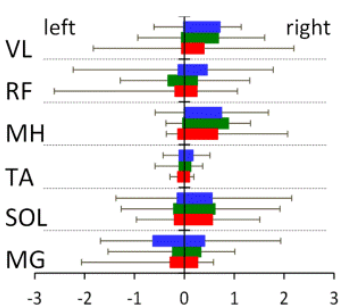

Threshold intensity
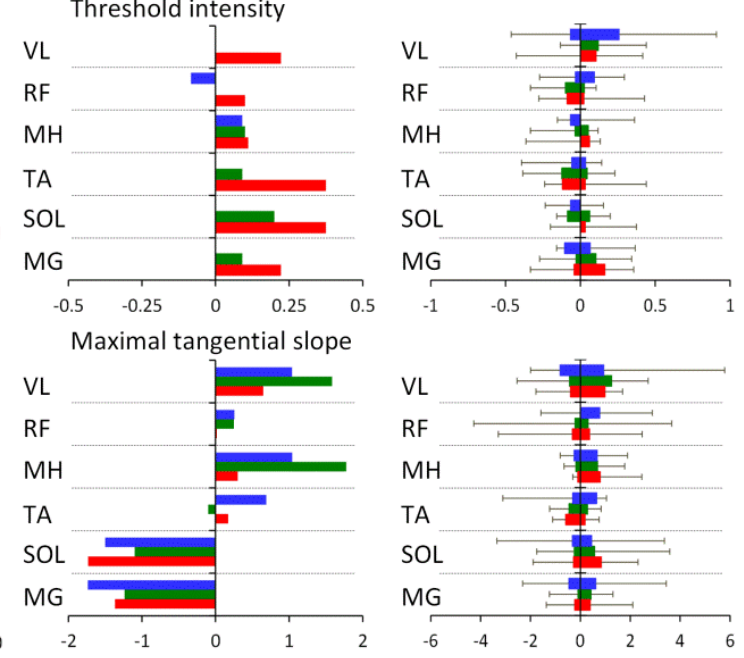

Left-Right difference (times)

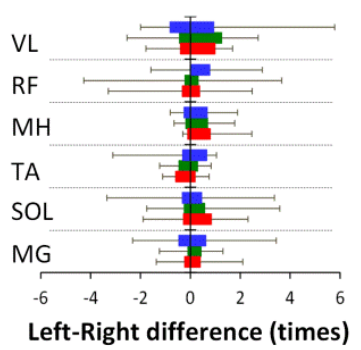

$A$ : recruitment curves of left and right muscles at each location of spinal

stimulation in one participant. Individual $(B)$ and pooled data $(C)$ illustrating the asymmetry level between left and right muscles in the plateau point magnitude (top), threshold intensity (middle), and maximal tangential slope (bottom). 


\title{
CHAPTER III
}

\section{DESCENDING INTERLIMB MODULATION OF LUMBOSACRAL NETWORK EXCITABILITY IN HUMAN}

\author{
$\underline{\text { Introduction }}$ \\ Recent studies of electrical spinal stimulation have revealed that \\ individuals with chronic, motor complete spinal cord injury $(\mathrm{SCl})$ retain the ability \\ to volitionally modulate spinal network excitability below the level of injury during \\ stimulation, resulting in the volitional movement of otherwise paralyzed limbs. \\ (Harkema, Gerasimenko et al. 2011, Angeli, Edgerton et al. 2014, Gerasimenko, \\ Lu et al. 2015). Of note is the rapid emergence of this ability following the \\ initiation of epidural stimulation, between 4-11 days in 3 participants (Angeli, \\ Edgerton et al. 2014). This finding suggests not only that spinal networks below \\ the level of injury maintain sufficient organization to integrate and execute \\ descending motor commands, but that some residual neural connectivity \\ between supraspinal centers and the lumbosacral circuitry likely exists in a \\ subset of individuals thought to have a motor complete SCI. Current clinical and \\ neurophysiological methods lack the sensitivity to measure, or appreciate \\ alterations to, pathway specific spinal transmission following neurological insult \\ or subsequent clinical rehabilitation.
}


Increased resolution in neurophysiological measurement of descending spinal projections is needed for the objective assessment of the extent of residual and/or newly formed pathways projecting to sublesional spinal networks following $\mathrm{SCl}$, and evaluation of their role in functional recovery. The current approach is based on non-invasive, transcutaneous electrical spinal stimulation, and designed to characterize the convergence of descending interlimb and peripheral afferent projections onto lumbosacral motor neurons. The proposed types of analyses are crucial to further our understanding of the structural and functional reorganization strategies of the human brain and spinal cord after neurological disorders or lesions. Additionally, the extent to which MMR methodology might be optimized for this purpose, and the development of analysis techniques allowing meaningful and contextual comparisons of conditioning effects in heteronymous motor pools has not been previously addressed.

The aim of the present study was to evaluate the neuromodulatory effects of descending interlimb pathways on the motor excitability of the lumbosacral circuitry, in order to characterize propriospinal influence on the excitability of lumbosacral motor pools projecting to proximal and distal leg muscles. We hypothesized that: 1) conditioning effects on the soleus MMR would be analogous to those observed in the $\mathrm{H}$-reflex, 2) and descending interlimb pathways can be engaged to modulate the amplitudes of multiple bilateral leg MMRs via ulnar nerve electrical stimulation in neurologically intact human participants. 


\section{Experimental Procedures}

\section{Participants}

15 volunteers ( 6 male and 9 female; mean \pm SD: age $29.1 \pm 5.3$ yrs., height $168 \pm 8 \mathrm{~cm}$, body mass $68.7 \pm 12.9 \mathrm{~kg}$ ) with no history of neurological injury or disease participated in the study. Written informed consent was obtained from all participants. The present study conformed to the standards set by the Declaration of Helsinki, and the procedures were approved by the University of Louisville (KY, USA) institutional review board the local ethics committee.

\section{Electromyography}

Surface EMG signals were recorded bilaterally in rectus femoris (RF), vastus lateralis $(\mathrm{VL})$, medial hamstrings $(\mathrm{MH})$ (semitendinosus), tibialis anterior (TA), medial gastrocnemius (MG) and lateral soleus (SOL) muscles, using bipolar pre-amplified surface electrodes (Motion Lab Systems, Baton Rouge, LA, USA) with fixed inter-electrode distance of $17 \mathrm{~mm}$. The reference electrode was placed over the middle $1 / 3$ of the left tibia. The EMG signals were differentially amplified using MA300 EMG System (Motion Lab Systems, Baton Rouge, LA, USA) with a band-pass filter of $10 \mathrm{~Hz}$ to $2 \mathrm{kHz}(-3 \mathrm{~dB})$. The EMG data were digitized at a sampling rate of $5000 \mathrm{~Hz}$.

Soleus $H$ - reflex

Soleus $\mathrm{H}$ - and M-responses were obtained using a custom-built constant current stimulator $(0-100 \mathrm{~mA}, 200 \mathrm{~V})$ to deliver single, $1 \mathrm{~ms}$, monophasic squarewave pulses every 6 seconds, via a monopolar surface electrode positioned over 
the posterior tibial nerve in the popliteal fossa. Recruitment curves for soleus $\mathrm{H}$ and M-responses were obtained as follows: stimulation began at subthreshold intensities and was increased at 1-2 mA increments until a plateau in the Mresponse was observed.

\section{Multisegmental muscle responses (MMRs)}

The same stimulator used for $\mathrm{H}$-reflex experiments was used to apply transcutaneous electrical spinal cord stimulation with the cathode (conductive rubber electrode, $18 \mathrm{~mm}$ diameter) placed on the skin between the spinous processes of the T10 and L2 vertebrae at the midline. Two $5 \times 9 \mathrm{~cm}$ selfadhesive electrodes (Pro-Patch, Taiwan) were used as anodes, placed bilaterally over the anterior superior iliac spine (Courtine, Harkema et al. 2007, Dy, Gerasimenko et al. 2010). Participants were placed in prone position on a hospital bed. Spinous processes were palpated and the interspinous spaces between the spinous processes of T10-T11, T11-T12, T12-L1, and L1-L2 were marked. Once the cathode was affixed over a specific interspinous space, the participant was moved to a supine position for the remainder of the study. Stimulation consisted of single, $1 \mathrm{~ms}$, monophasic square-wave pulses delivered every 6 seconds. Electrical stimulation began at an intensity which was subthreshold for all muscles, and then increased at $2 \mathrm{~mA}$ increments, with 3 stimuli presented at each increment up to $100 \mathrm{~mA}$, or the maximum tolerable intensity. Recruitment curves were then generated by plotting the MMR amplitude as a function of stimulus intensity. Recruitment threshold for a particular motor pool varied predictably during spinal stimulation at different 
rostro-caudal locations (figure 10). More rostral stimulation sites resulted in recruitment of more rostrally located motor pools (i.e., VL) at lower stimulation intensities, while more caudal stimulation sites similarly resulted in earlier recruitment of caudal motor pools (i.e., SOL). In each participant example, one location of stimulation resulted in a range of stimulus intensities at which submaximal MMRs could be evoked in both proximal (VL) and distal (SOL) motor pools. In the participant represented in panel A, this was identified as the T10T11 level, while the participant represented in panel B demonstrates this recruitment pattern at T11-T12. As such, location of stimulation for conditioning experiments was determined by the recruitment pattern of MMRs, as opposed to using a bony landmark for site selection. As a result, the location of stimulation selected for conditioning experiments varied across patients, with spinal stimulation provided at T9-T10 $(n=1)$, T10-T11 $(n=8)$, T11-T12 $(n=7)$, and T12-L1 $(n=1)$.

\section{Ulnar nerve stimulation}

Ulnar nerve stimulation was applied just proximal to the elbow on the ventromedial surface of the arm via bipolar stimulating electrode of fixed interelectrode distance of $30 \mathrm{~mm}$ (MLADDF30, AD Instruments, New Zealand) using a constant current stimulator (DS5, Digitimer®, UK). Motor threshold for the abductor digiti minimi was identified, and the conditioning stimulation was delivered at 3x motor threshold intensity (Meinck and Piesiur-Strehlow 1981, Gorodnichev, Pivovarova et al. 2012), via three $1 \mathrm{~ms}$ square wave pulses with inter-stimulus interval of $1 \mathrm{~ms}$. 


\section{Experimental protocols}

To confirm the suitability of MMRs for conditioning experiments, the effects of ulnar nerve stimulation on the amplitudes of the soleus $\mathrm{H}$-reflex and MMR were compared in four participants. The test $\mathrm{H}$-reflex amplitude was $20-$ $30 \%$ of M max (Crone, Hultborn et al. 1990), then the amplitude of the MMR was matched.

MMR recruitment curves were obtained from multiple rostro-caudal locations in 11 participants. In order to standardize the procedure across participants, location of stimulation used in conditioning experiments was subsequently selected based on order of MMR recruitment (i.e. the approximate spinal level of stimulation, as opposed to a fixed location relative to a vertebral level). Further, to optimize the technique, we studied the conditioning effects of ulnar nerve stimulation on MMRs of varying magnitude in 8 participants. Conditioning bouts were conducted at multiples of the MMR threshold intensity $(x 1.2, x 1.4, x 1.6, x 1.8, x 2.0)$.

The time-course of ulnar stimulation induced facilitation was studied, using condition-test intervals of $40,50,60,70,90,110,125,140,160$, and $190 \mathrm{~ms}$. All experimental bouts began with spinal stimulation only, at a rate of one every 6 seconds. Following collection of 10 unconditioned MMRs, conditioning stimulation at the ulnar nerve was initiated, with condition-test-intervals presented randomly. Each condition-test interval was presented a total of 10 times, along with at least 10 interspersed controls. Following completion of the conditioning stimuli, 10 additional unconditioned MMRs were recorded. 


\section{Data analysis}

The magnitude of $\mathrm{H}$-reflexes and MMRs were quantified as the peak-topeak amplitude of the evoked responses. Recruitment curves were modeled by a 3-parameter logistic (3PL) non-linear mixed effects models. Subject- and muscle-specific estimates of the recruitment curve parameters - (1) the maximum MMR amplitude (MMRmax), (2) MMR amplitude at the maximum rate of recruitment (RRmax), and (3) the midpoint of the curve were generated from the best linear unbiased predictions from the mixed effects model.

Response amplitudes from conditioning experiments were modeled with a nested, mixed effects analysis of covariance (ANCOVA). The primary fixed effects were the latency of the condition-test interval (unconditioned, 40ms, 50ms, etc.) and muscle, a 12-level factor. The best linear unbiased predictions estimates of MMRmax, RRmax, and the recruitment curve midpoint were also included as fixed effects in the model to adjust comparisons of control and conditioned responses for the features of each subject's recruitment curve. Twoway interactions between the muscle fixed effect and each other fixed effect were included. The model intercepts were included as random effects within muscle within subject, so that linear contrasts comparing control and conditioned responses were conducted within muscle and subject. Conditioned responses were compared between left and right sides via linear contrasts applied to the fitted linear mixed effects model.

A targeted analysis of the relationship between conditioning effects and the magnitude of stimulation relative to RRmax was conducted on 8 subjects 
(see methods). A linear mixed effects model was employed to test the relationship between the magnitude of the conditioning effect and the intensity of stimulation relative to RRmax. Analyses were conducted in the open-source R software package (R Core Team (2015). R: A language and environment for statistical computing. R Foundation for Statistical Computing, Vienna, Austria. http://www.r-project.org/) using the nlme library for mixed effects models (Pinheiro 2000). All significance tests were conducted at the .05 level.

\section{$\underline{\text { Results }}$}

Ulnar nerve stimulation modulates the amplitude of soleus $\mathrm{H}$-reflex and MMR H-reflexes and MMRs displayed similar characteristics in recruitment curves and modulation via ulnar nerve stimulation. Recruitment curves of the $\mathrm{H}$ reflex (figure 11A) and MMR (figure 11B) were recorded in the SOL during the same experimental session in four participants. Stimulation of the ulnar nerve prior to induction of either the H-reflex or MMR in the SOL resulted in an increase in response amplitude (figure 11C, D). Facilitation of the reflex responses began at condition-test intervals of $50 \mathrm{~ms} / 60 \mathrm{~ms}$, and reached a maximum at conditiontest intervals of $100 \mathrm{~ms} / 110 \mathrm{~ms}$, for the $\mathrm{H}$-reflex and MMR respectively, and was observed in all participants tested (figure 11E, F).

Multi-segmental muscle responses (MMRs), evoked in multiple bilateral leg muscles via transcutaneous spinal cord stimulation, are known to share several properties with the well-studied H-reflex, including (i) suppression of the responses by tendon vibration (Courtine, Harkema et al. 2007, Minassian, Persy et al. 2007, Dy, Gerasimenko et al. 2010) (ii) the presence of reciprocal inhibition 
between antagonists (Minassian, Persy et al. 2007, Hofstoetter, Minassian et al. 2008), (iii) facilitation of the responses during voluntary agonist contraction (Minassian, Persy et al. 2007, Hofstoetter, Minassian et al. 2008), (iv) task- and phase- dependent modulation during standing and gait (Courtine, Harkema et al. 2007, Hofstoetter, Minassian et al. 2008, Dy, Gerasimenko et al. 2010), and (v) facilitation when conditioned by transcranial magnetic stimulation over the motor cortex (Wolfe, Hayes et al. 1996, Roy, Bosgra et al. 2014).

\section{Ulnar nerve stimulation-induced modulation of bilateral leg MMRs}

Significant facilitation of bilateral leg MMRs in response to ulnar nerve stimulation was observed in all analyzed muscles in all participants as exemplified in figure 3A. Group analyses demonstrated a pattern of facilitation in all muscles, beginning as early as the $40 \mathrm{~ms}$ condition-test interval-the earliest interval tested, and exhibiting at least two peaks: the first and largest occurring at the $90 \mathrm{~ms}$ condition-test interval $(p<.0001)$, and the second at the $140 \mathrm{~ms}$ condition-test interval $(p<.001)$ (figure 12B). Across muscles, MH, VL, MG, and SOL demonstrated similar magnitudes of facilitation for a given condition-test interval, while RF and TA demonstrated a relatively smaller magnitude response to conditioning stimuli (figure 12B). In addition, significant differences were found between ipsi- and contralateral muscles at the $60 \mathrm{~ms}$ condition-test interval in $\mathrm{MH}$, VL, MG, and SOL, wherein the muscles ipsilateral to the side of ulnar nerve stimulation demonstrated a greater magnitude of facilitation $(p<.01)($ figure 12B). Little to no modulation was seen at this condition-test interval in RF and TA. Additionally, significant left-right differences were also found for $\mathrm{MH}$ at condition- 
test intervals of 90 and $110 \mathrm{~ms}$, and for $\mathrm{VL}$ at condition-test intervals of 50,70 , 110 , and 160ms $(p<.01)$ (figure 12B).

Magnitude of conditioning effect was related to rate of recruitment

Conditioning experiments at multiple spinal stimulation intensities allowed observation of the conditioning effect on MMRs of different amplitudes (figure 13). When comparing the stimulation intensities used in the conditioning experiments (figure 13A) to the rate of recruitment at the point on the recruitment curve corresponding to those stimulation intensities (figure 13B), it can be seen that the greatest magnitude of conditioning always occurred at or near the maximum rate of recruitment.

In order to identify the test responses resulting in the greatest absolute magnitude of facilitation, the amplitude of unconditioned MMRs was subtracted from that of the conditioned MMRs, and divided by the RRmax, which was defined as the MMR amplitude at the point in the recruitment curve where the maximum rate of recruitment was observed (figure 14). Normalization of the MMR amplitudes revealed that the magnitude of facilitation was related to the size of the test MMR $(p<.01)$, with the effect peaking at test reflex amplitudes equal to RRmax (figures 14, 15).

\section{Discussion}

The present study establishes the utility and sensitivity of transcutaneous spinal stimulation in human neurophysiological studies as a tool for the objective characterization of the neuromodulatory effects of a specific descending motor system on multiple bilateral lumbosacral motor pools. Facilitation of MMRs 
recorded from bilateral knee and ankle muscles, resulting from the convergence of descending interlimb and lumbosacral afferent volleys is demonstrated. Criteria were identified for standardization and optimization of MMR methodology, and analyses methods are presented which allow physiologically valid comparisons of the conditioning effects among individual motor pools.

Descending interlimb modulation of leg MMRs

Significant facilitation of leg MMRs was elicited by stimulation of the ulnar nerve (figures 12,13). The evidence for descending interlimb modulation of the reflex excitability of motor neurons within the lumbosacral enlargement to date has been largely limited to the soleus muscle H-reflex (Piesiur-Strehlow and Meinck 1980, Meinck and Piesiur-Strehlow 1981, Delwaide and Crenna 1984, Kagamihara, Hayashi et al. 2003), representing just one unilateral motor pool (Sharrard 1964, Kendall, McCreary et al. 1983).

The extent to which descending excitation from unilateral upper limb afferents converges with lumbosacral afferent input to modulate bilateral spinal motor neuron excitability throughout the lumbosacral enlargement has not previously been reported. The current results extend the existing findings, demonstrating the convergence of descending and afferent inputs in bilateral motor pools spanning the lumbosacral enlargement.

Neural pathways mediating descending modulation of spinal motor excitability

The overall time-course of facilitation in the current study, with facilitation beginning at $40-50 \mathrm{~ms}$ and peaking at $90 \mathrm{~ms}$, followed by a second peak in facilitation at 140ms, was observed in all muscles studied (figure 12). The 
conditioning effect first reached statistical significance at the $40 \mathrm{~ms}$ condition-test interval in $\mathrm{VL}$ and SOL, and at the $60 \mathrm{~ms}$ condition-test interval was significant in all muscles except TA (figure 12B). After accounting for afferent conduction, this represents a central conduction time of $\sim 25-45 \mathrm{~ms}$, consistent with a fast conducting, directly descending propriospinal tract (Piesiur-Strehlow and Meinck 1980, Meinck and Piesiur-Strehlow 1981, Zehr, Collins et al. 2001, Butler, Godfrey et al. 2016). Transcutaneous stimulation of dorsal root afferents at the C4 and T9 vertebral levels has been shown to result in the same facilitation pattern when conditioning the $\mathrm{H}$ reflex, with $\mathrm{T} 9$ stimulation producing the effect at shorter condition-test intervals, implying a directly descending propriospinal pathway at short latencies (Meinck and Piesiur-Strehlow 1981). Short latency facilitation $(\leq 60 \mathrm{~ms})$ was of significantly greater magnitude in ipsilateral leg muscles (figure 12), consistent with previous propriospinal studies both in the cat (Schomburg, Meinck et al. 1978) and in humans (Meinck and Piesiur-Strehlow 1981, Sarica and Ertekin 1985, Butler, Godfrey et al. 2016). The long ascending and descending propriospinal pathways, first identified and extensively studied in animals, are known to be important in forelimb-hind limb coordination (Lloyd and Mclntyre 1948, Gerasimenko, Musienko et al. 2009, Cowley, Zaporozhets et al. 2010). Neurophysiological studies of human interlimb reflexes provide mounting evidence for the direct propriospinal mediation of task-specific modulation of motor excitability in arm and leg muscles during repetitive tasks requiring interlimb coordination (Suzuki, Nakajima et al. 2014, Sasada, Tazoe et al. 2016, Suzuki, Nakajima et al. 2016). 
Several have noted the seemingly fixed pattern of facilitation in the soleus H-reflex in response to stimulation of a wide variety of both ipsi- and contralateral upper limb and trunk cutaneous and mixed peripheral nerves (Meinck and Piesiur-Strehlow 1981, Delwaide and Crenna 1984, Zehr, Collins et al. 2001, Kagamihara, Hayashi et al. 2003, Zehr, Frigon et al. 2004). Facilitation observed at longer condition-test intervals $(\geq 100 \mathrm{~ms})$, likely reflects oligosynaptic, supraspinally mediated reflex pathway(s) (Delwaide and Crenna 1984, Delwaide and Schepens 1995, Kennedy and Inglis 2001, Kagamihara, Hayashi et al. 2003). As such, it seems likely that reflex pathways involving multiple descending tracts may be represented in the time-course of the facilitation, with directly descending propriospinal pathways responsible for the early facilitation, and facilitation at longer latencies attributable to a startle response (Meinck and Piesiur-Strehlow 1981, Dragert and Zehr 2009). A subpopulation of long descending propriospinal neurons located in the cervical enlargement, with ascending collateral projections to the reticular formation and/or cerebellum has been described in mammals (Skinner, Nelson et al. 1989, Verburgh, Kuypers et al. 1989), and would be well suited to mediate both the short- and long latency facilitation observed.

\section{Magnitude of MMR modulation is dependent upon rate of recruitment}

The rate of recruitment of an individual muscle's MMR was significantly related to the presence and magnitude of modulation (figures 13-15). MMRs from all muscles exhibited the greatest magnitude of facilitation when the amplitude of the test MMR was at or near RRmax (figures 13-15). This relationship allows 
normalization of conditioning results relative to the proportion of each motor pool excited by the test stimulus. The maximum rate of recruitment, which was quantified as the maximum slope of the recruitment curve, has been shown to vary across motor pools (Sayenko, Atkinson et al. 2015). The rate of recruitment can be understood as the sensitivity of a motor pool to incremental increases in excitatory afferent input. It was previously demonstrated that $\mathrm{MH}, \mathrm{VL}, \mathrm{MG}$, and SOL consistently exhibit greater maximum rates of recruitment as compared with RF and TA, with the same muscles exhibiting the greatest magnitude of facilitation in response to ulnar nerve stimulation in the current study (figure 12).

The current results could be interpreted as evidence that significant overlap exists in the projection patterns of both the descending interlimb and peripheral afferent excitatory inputs directly onto motor neurons within a given motor neuron pool. However, the consistent nature and extent of facilitation observed in heteronymous muscles when the excitability of the lumbosacral circuitry was altered by transcutaneous spinal stimulation is in contrast to that observed in a similar study in which descending reflexes were identified in quiescent knee and ankle flexor and extensor muscles via modulation of baseline EMG. Under these conditions, less than $25 \%$ of all muscles studied demonstrated descending interlimb modulation (Butler, Godfrey et al. 2016). As such, it seems more likely that descending interlimb and multisegmental afferent inputs are integrated by pre-motor interneurons, thus modulating the excitability state of the entire motor neuron pool. Pre-motor convergence has been reported in studies evoking cortical and peripheral volleys, with the short lumbar 
propriospinals located in the rostral lumbar segments implicated (MarchandPauvert, Simonetta-Moreau et al. 1999, Pierrot-Deseilligny 2005).

\section{Optimization of stimulation location}

Here the location for transcutaneous spinal stimulation was selected based on the order of recruitment of spinal motor pools. Specifically, we selected a stimulation location in which activation thresholds of proximal muscles (L2-L4) were observed at lower stimulation intensities relative to distal leg muscles (S1S2) (figure 10). This ensured that stimulation was applied along the most rostral portion of the lumbosacral enlargement, in order to standardize the procedure across participants, as well as to minimize the possibility of stimulation of mixed peripheral nerves from the most rostral segments as they descend outside the spinal cord (Minassian, Persy et al. 2007, Troni, Di Sapio et al. 2011, Sayenko, Atkinson et al. 2015).

Previous studies of MMR modulation have selected the site of stimulation location based on bony vertebral landmarks, typically the T11-T12 interspinous space (Courtine, Harkema et al. 2007, Hofstoetter, Minassian et al. 2008, Dy, Gerasimenko et al. 2010). Anatomical variability in the level of termination of the spinal cord with reference to the spinal vertebrae has been demonstrated (Troni, Di Sapio et al. 2011), so use of a bony landmark likely leads to variability with respect to order of recruitment of MMRs across participants, as has been demonstrated previously (Troni, Di Sapio et al. 2011, Sayenko, Atkinson et al. 2015) and in the current study.

\section{Limitations}


While there are several advantages in the use of MMRs to monitor motor neuron excitability in electrophysiological studies, some limitations remain. Notably, in H-reflex studies, the M-wave provides a measure of the consistency of the test response. Since transcutaneous spinal stimulation does not produce an M-wave, there is no simple method for monitoring the unconditioned MMR amplitude throughout a conditioning bout.

Although we sought to assess descending propriospinal influence on the lumbosacral motor circuitry, the current data do not allow elimination of other descending tracts as potential contributors to the observed effects. However, ongoing studies on the influence of other major descending tracts on lumbosacral motor neuron excitability will improve our understanding of the facilitation/inhibition profiles of different descending tracts. As our methods were designed to be easily transferred to a clinical population with limited mobility, a supine testing position was ideal. However, this limits our ability to interpret the behavioral relevance of the interlimb modulation observed.

\section{Future directions}

There is a need for more sensitive measures of residual and subclinical neural connectivity following $\mathrm{SCl}$ than are currently available. Assessment of descending pathway viability after disease/injury of the central nervous system can provide increased resolution regarding the nature and severity of injury with respect to the descending motor systems. Studies of epidural spinal cord stimulation following chronic motor-complete $\mathrm{SCl}$ suggest that sub-clinical neural influence on lumbosacral motor pools exists following even severe $\mathrm{SCl}$ (Angeli, 
Edgerton et al. 2014). The current study, in conjunction with ongoing studies of other descending motor pathways, including vestibulospinal, reticulospinal, and corticospinal tracts, can reveal new insights into the influence of descending tracts on the lumbosacral motor circuitry, as well as their potential reorganization to restore functional cortical connections to the lumbosacral circuitry following $\mathrm{SCl}$. Identification of residual and/or de novo descending pathways capable of influencing spinal excitability, as well as the neuronal populations involved, is necessary to further our understanding of the mechanisms involved in the spinal stimulation-dependent restoration of volitional motor function after SCl (Angeli, Edgerton et al. 2014). Further, identification of residual descending influence in clinical populations can reveal potential therapeutic targets to researchers and clinicians.

\section{$\underline{\text { Conclusions }}$}

In summary, the current work uncovers the capacity of the descending interlimb pathways to modulate the excitability of spinal motor pools throughout the lumbar enlargement. These findings reveal the multi-segmental and bilateral influence of cervical afferents on lumbosacral motor neuron pools, and support the existence a descending propriospinal system in humans. As compared with previous studies, the methods described allow detection of descending interlimb influence on motor excitability, providing quantitative assessment of descending spinal transmission after neurological insult. As neurological injuries are frequently asymmetric in presentation, the described techniques are of value to studies evaluating the degree of symmetry of intact descending motor projections 
and their relationship to functional abilities and/or limitations post injury. Further, we demonstrated that optimization of the MMR methodology could maximize the sensitivity of this approach, allowing intra- and interlimb comparisons among individual muscles, which can be interpreted within the context of each motor pool's relative excitability state. 
Figure 10. MMR recruitment curves for the $\mathrm{VL}$ and $\mathrm{SOL}$ muscles in two representative participants

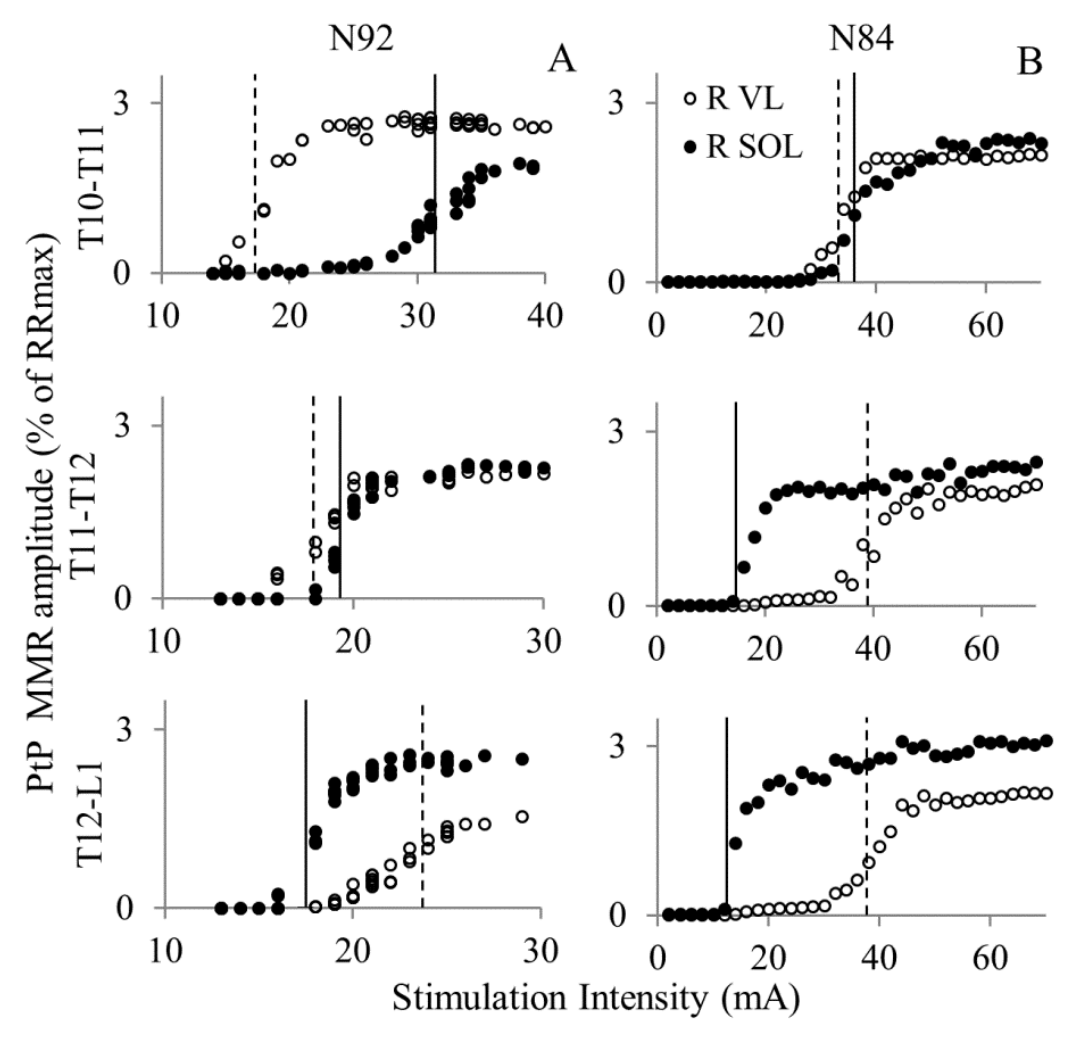

MMR recruitment curves for the $\mathrm{VL}$ and $\mathrm{SOL}$ muscles in two representative participants (A, B) at three different spinal stimulation locations: T10-T11, T11T12, and T12-L1. The vertical lines indicate the RRmax for VL (hashed) and SOL (solid). 
Figure 11. Conditioning of soleus $\mathrm{H}$-reflex and MMR via ulnar nerve stimulation
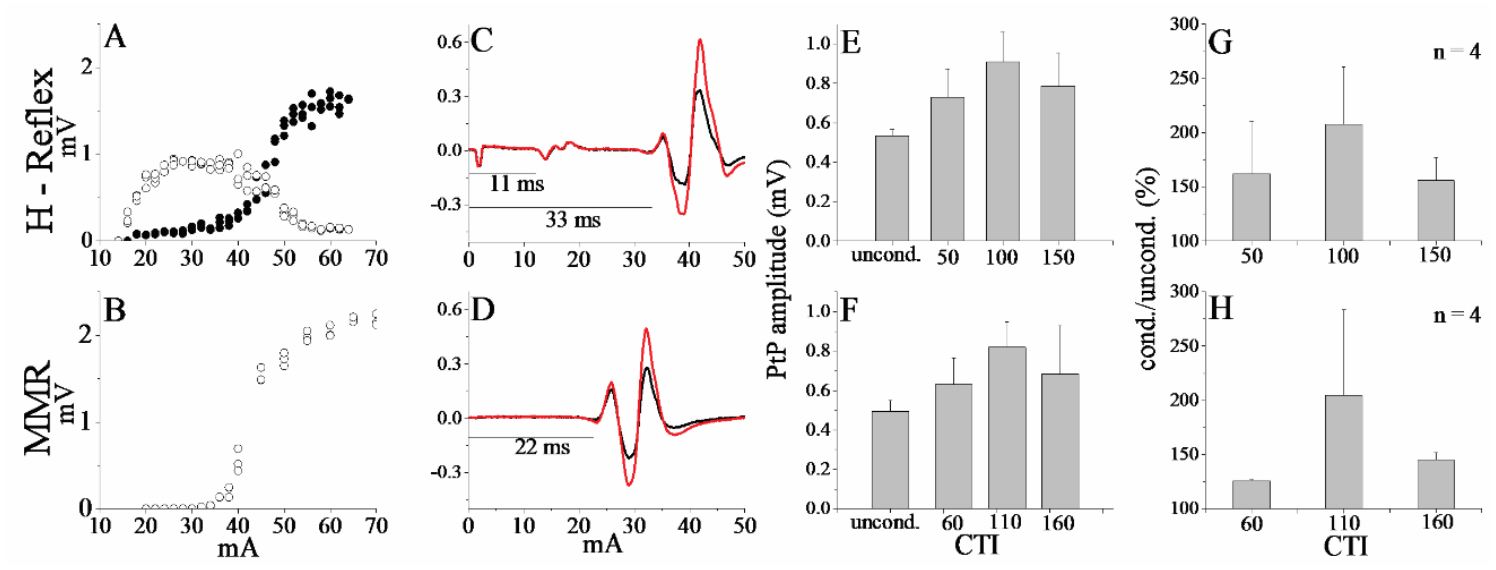

Recruitment curves for the H-reflex (A) and MMR (B). Empty circles represent reflex responses and filled circles represent direct motor responses. Average of 10 unconditioned (black) and 10 conditioned (red) H-reflexes (C) and MMRs (D) recorded in the right soleus of a representative participant. Conditioning results over a range of condition-test intervals (CTIs) for H-reflex (E) and MMR (F) of the representative participant. Group conditioning results over a range of CTIs for $\mathrm{H}$ reflex $(G)$ and MMR $(H)$. 
Figure 12. Conditioning effect of ulnar nerve stimulation on leg MMRs

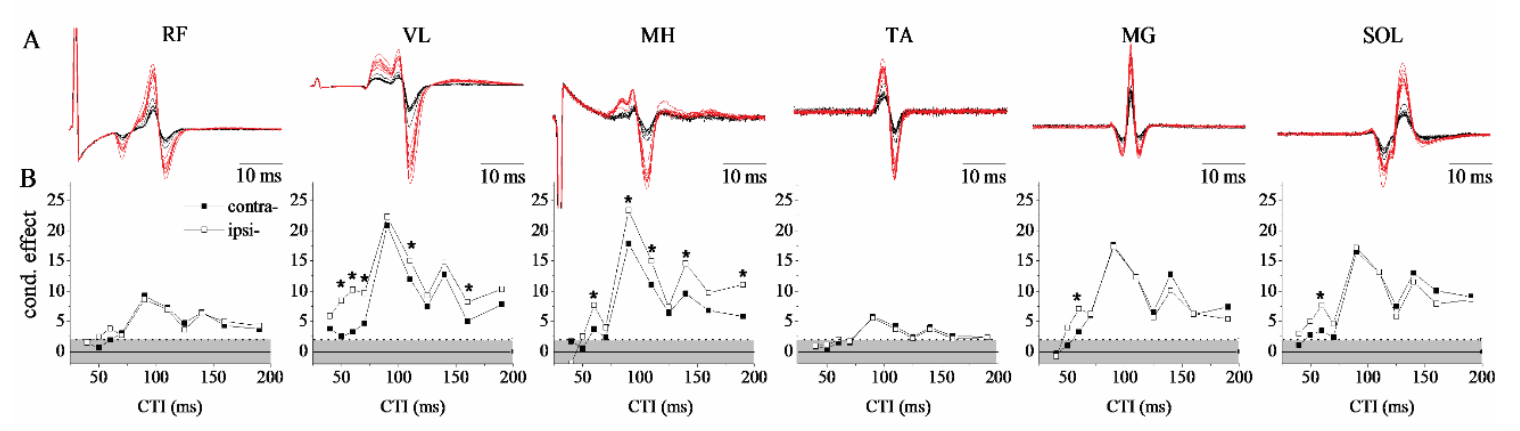

(A) Unconditioned (black) and Conditioned (red) MMRs (10 responses each) for

$\mathrm{RF}, \mathrm{VL}, \mathrm{MH}, \mathrm{TA}, \mathrm{MG}$ and $\mathrm{SOL}$ in a representative research participant. (B) Group data $(n=15)$ detailing the conditioning effect by muscle and condition-test interval (CTI). Values above the shaded area are statistically significant. Significant differences between left (contralateral) and right (ipsilateral) muscles are indicated by an asterisk. 
Figure 13. Relationship between MMR rate of recruitment and conditioning effects

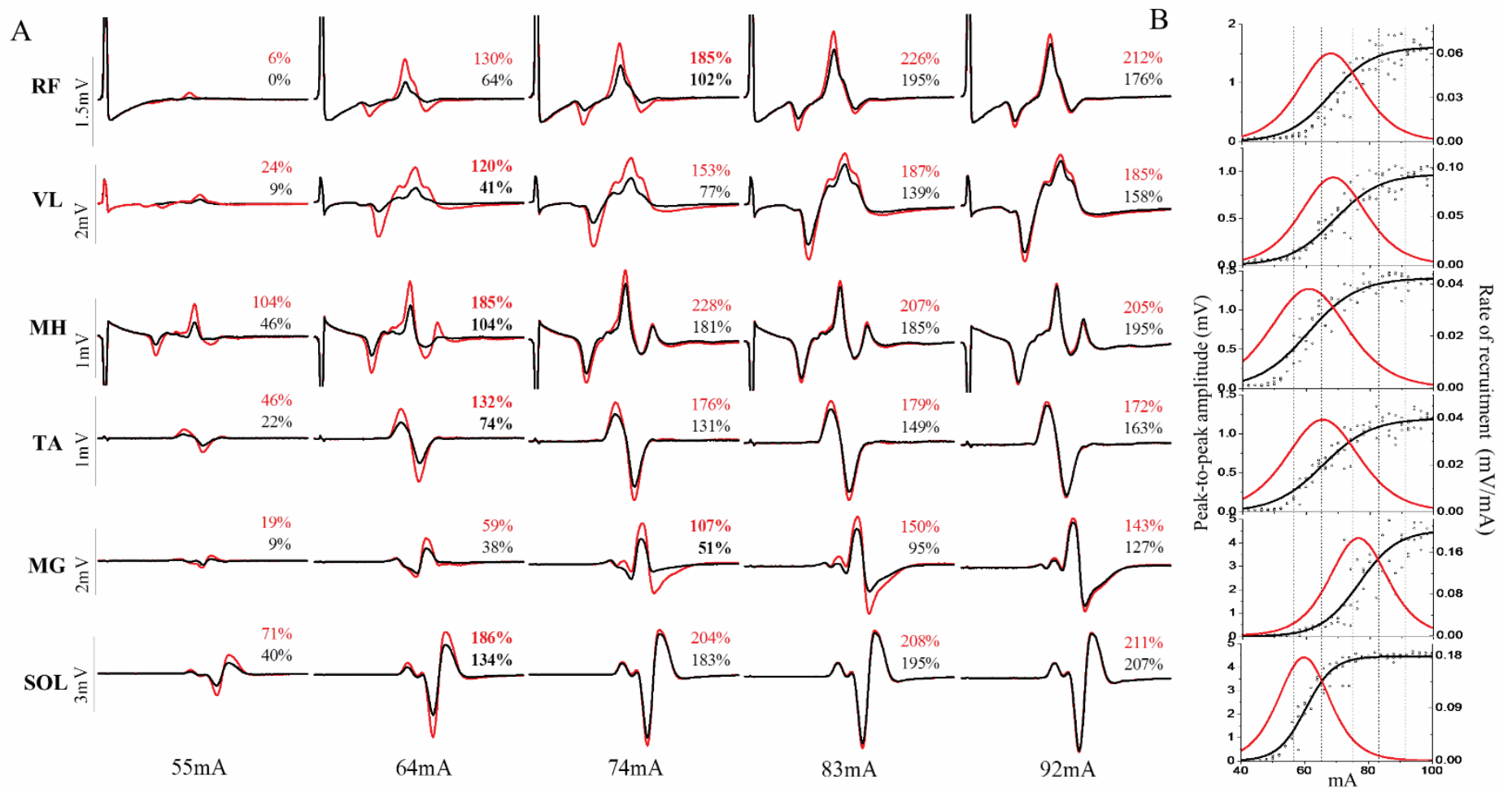

(A) Average of 10 unconditioned (black) and 10 conditioned (red) MMRs from 6 different muscles at 5 different spinal stimulation intensities in a representative participant. The condition-test interval is $90 \mathrm{~ms}$. Amplitude of the unconditioned and conditioned MMRs, expressed as a percentage of RRmax are given for each MMR. (B) MMR Recruitment curves for each muscle are plotted (empty circles). The black line represents the estimated recruitment curve. The red line gives the estimated rate of recruitment (right y-axis). Vertical dashed lines denote the spinal stimulation intensities of each conditioning bout depicted in panel A. 
Figure 14. Conditioning results from the individual example given in figure 13

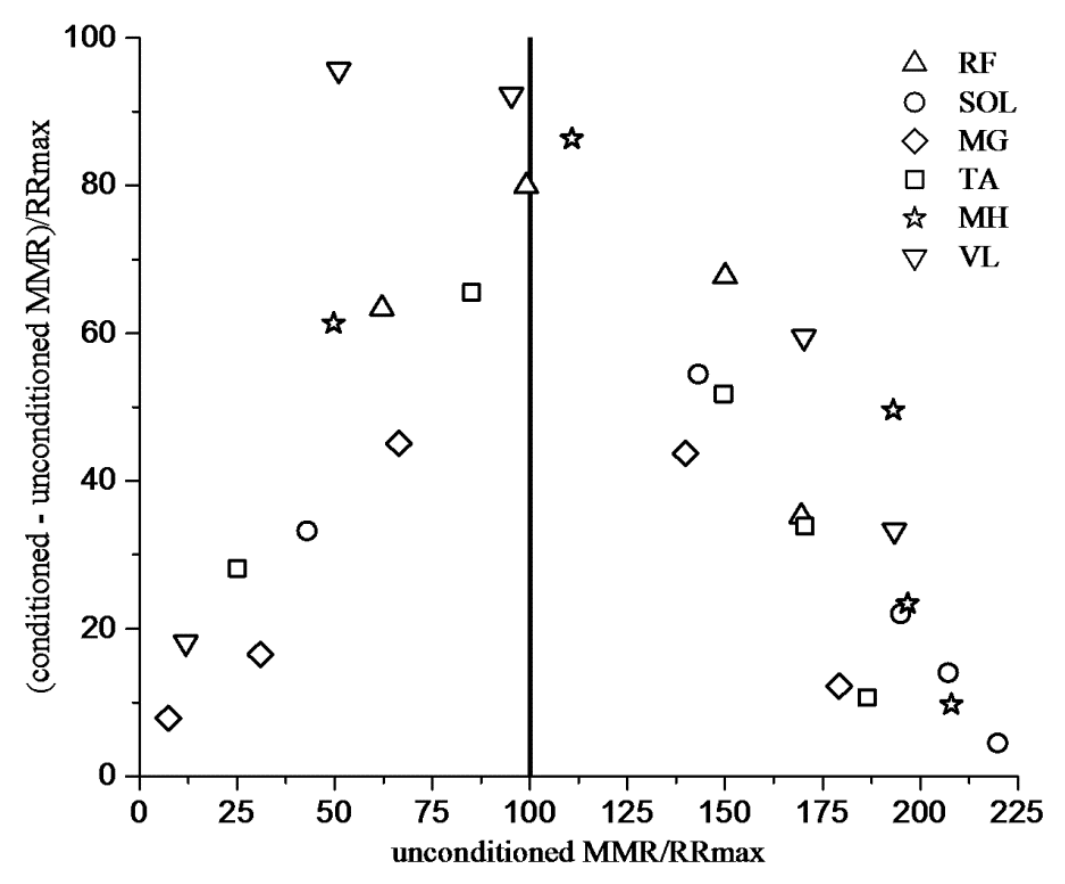

Control amplitudes were subtracted from conditioned amplitudes, normalized to RRmax and plotted against the amplitude of the unconditioned MMR, expressed as a percentage of RRmax. 
Figure 15

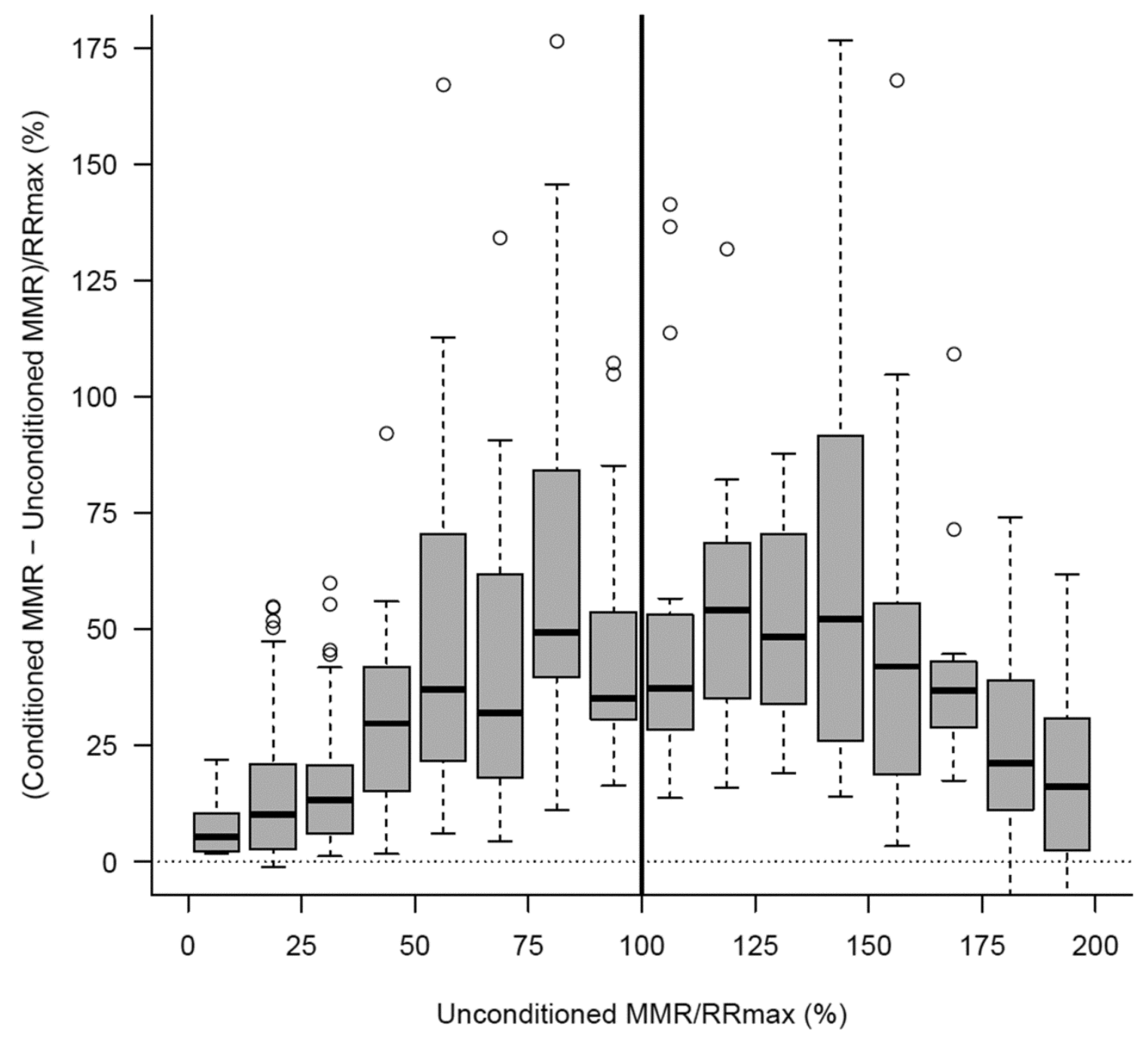

Group data $(n=15)$ illustrating the magnitude of conditioning as a function of unconditioned MMR amplitude, both normalized to RRmax. Bin widths for stimulation intensity are $12.5 \%$ RRmax. Open circles represent outlying conditioning responses. Vertical Black line denotes RRmax 


\section{CHAPTER IV}

\section{INTERLIMB REFLEXES REVEAL DESCENDING MODULATION OF SPINAL EXCITABILITY AFTER HUMAN SPINAL CORD INJURY}

\section{Introduction}

Spinal Cord Injury (SCl) in humans may result from a variety of different injury mechanisms, resulting in variable muscle paralysis and/or paresis based on the location and severity of injury. One of the primary determinants of rehabilitative prognosis is the existence and prevalence of motor function below the level of injury (Kirshblum and O'Connor 1998, 2008). Currently, clinicians frequently rely on subjective assessment of residual motor function in a limited set of muscles for determination of spared motor function after SCl (Kirshblum, Waring et al. 2011).

Both neurophysiological (Dimitrijevic, Hsu et al. 1992, Sherwood, Dimitrijevic et al. 1992) and anatomical evidence (Bunge, Puckett et al. 1993, Kakulas 1999) has long suggested that residual descending connectivity between supraspinal structures and the intact spinal circuitry below the level of lesion, which cannot be detected upon clinical examination, exists in a subset of individuals clinically diagnosed as having a motor complete lesion. However the clinical relevance of these findings seemed insignificant until reports of human 
epidural spinal stimulation after chronic, motor complete SCI provided compelling evidence that these residual pathways may be capable of mediating volitional movement when the functional state of spinal circuitry is enhanced (Harkema, Gerasimenko et al. 2011, Angeli, Edgerton et al. 2014).

Despite the known heterogeneity of injury mechanism and subsequent clinical presentation associated with human SCI (Behrman, Ardolino et al. 2012), until recently little has been done to investigate the viability of specific descending spinal tracts after human $\mathrm{SCl}$, with the exception of the corticospinal tracts. Recent studies have demonstrated that residual descending influence can be electrically evoked through corticospinal, vestibulospinal, and descending interlimb pathways, however response rates in motor complete $\mathrm{SCl}$ individuals has been minimal in muscles innervated by the lumbosacral enlargement (Barthelemy, Willerslev-Olsen et al. 2015, Butler, Godfrey et al. 2016, Squair, Bjerkefors et al. 2016). As discussed by the authors, electrically evoked responses in quiescent muscles may be difficult to obtain even in neurologically intact individuals, with the problem being compounded after SCl. While volitional contraction may improve response rates, spinal cord injured individuals with motor complete injuries are unable to volitionally activate muscles below the level of injury. As an alternative, a condition-test paradigm allows for the effect of descending volleys to be investigated in sub-lesional motor pools in which the excitability is electrically modulated. As such, we developed electrophysiological methods for the comprehensive investigation of descending tract-specific 
influence on lumbosacral motor neuron excitability utilizing transcutaneous spinal electrical stimulation (Atkinson et al., 2016, submitted).

In the current study, we sought to identify descending propriospinal influence on lumbosacral motor excitability after chronic SCl in human. We assessed descending propriospinal function through the induction of interlimb reflexes, thought to be mediated by propriospinal neurons (Atkinson et al., 2016, submitted)(Meinck and Piesiur-Strehlow 1981, Zehr, Collins et al. 2001), in order to evaluate descending influence on spinal motor excitability. In order to assess the relevance of residual descending interlimb inputs after SCI to motor recovery, volitional muscle activation of bilateral leg muscles was also evaluated.

\section{Methods}

\section{Participants}

18 volunteers ( 16 male and 2 female; mean \pm SD: age $29.6 \pm 7.1$ years, time since injury $4.7 \pm 3.1$ years) participated in the study. Only individuals with upper motor neuron lesion were included, with the level of injury as follows:

$\mathrm{C} 2=1, \mathrm{C} 4=4, \mathrm{C} 5=3, \mathrm{C} 6=1, \mathrm{C} 7=1, \mathrm{~T} 2=3, \mathrm{~T} 4=4$, and $\mathrm{T} 6=1$. The Participants' injury severity was assessed clinically via the International Standards for the Neurological Classification of Spinal Cord Injury (ISNCSCI) American Spinal Injury Association Impairment Scale (AIS). According to the exam, subjects were classified as motor complete (AIS A, n=5), motor complete and sensory incomplete (AIS B, n=4), or motor and sensory incomplete (AIS C, n=4, and AIS D, n=5) (Table 5). Written informed consent, approved by the University of Louisville (KY, USA) institutional review board, was obtained from all participants. 
Functional Neurophysiological Assessment (FNPA)

In order to objectively identify the presence of volitional muscle activation following $\mathrm{SCl}$, participants' ability to generate volitional activation in muscles innervated below the level of lesion was assessed via functional neurophysiological assessment. As descried in detail elsewhere, assessment is based on a rigidly administered, standardized protocol allowing reliable characterization of the extent of volitional motor control, as well the existence of subclinical descending influence on spinal motor excitability below the lesion. Briefly, participants are positioned in supine, and instructed in the performance of standardized movement attempts ( 3 trials each) targeting each of 16 muscles recorded bilaterally, chosen to represent motor pools throughout the cervical, thoracic, and lumbosacral segments. Reinforcement maneuvers and tonic vibratory reflex are assessed for determination of residual descending influence in the absence of volitional muscle activation, identifying 'discomplete' injury, in addition to patellar and Achilles tendon stretch reflex tests, and assessment of ankle clonus.

Surface EMG signals were recorded bilaterally from sternocleidomastoid (SCM), upper trapezius (UT), biceps brachii (BB), triceps brachii (TB), extensor carpi radialis (ECR), flexor digitorum profundus (FDP), abductor digiti quniti (ADQ), external intercostal (IC6 - th $^{\text {th }}$ intercostal space), rectus abdominus (RA), erector spinae (EST10 - lateral to T10 spinous process), rectus femoris (RF), vastus lateralis $(\mathrm{VL})$, medial hamstrings ( $\mathrm{MH}$-semitendinosus), tibialis anterior (TA), extensor digitorum longus (EDL) and lateral soleus (SOL), in individuals 
with injuries above T2. In individuals with injuries below the T2 neurological level, and thus with no impairment of upper limb musculature, BB, TB, ECR, FDP, and $A D Q$, were replaced with external oblique (EO), erector spinae at L2 (ESL2), peroneous longus $(\mathrm{PL})$, medial gastrocnemius $(\mathrm{MG})$, and flexor hallucis brevis (FHB) for greater resolution in the motor segments below the lesion. Recordings were conducted using bipolar pre-amplified surface electrodes (Motion Lab Systems, Baton Rouge, LA, USA) with fixed inter-electrode distance of $17 \mathrm{~mm}$. The reference electrode was placed over the middle $1 / 3$ of the left tibia. The EMG signals were differentially amplified using MA300 EMG System (Motion Lab Systems, Baton Rouge, LA, USA) with a band-pass filter of $10 \mathrm{~Hz}$ to $2 \mathrm{kHz}(-3$ dB). The EMG data were digitized at a sampling rate of $2000 \mathrm{~Hz}$.

\section{Multisegmental muscle responses (MMRs)}

A custom-built constant current stimulator $(0-100 \mathrm{~mA}, 200 \mathrm{~V})$ was used to deliver single, $1 \mathrm{~ms}$, monophasic square-wave pulses to the cathode (conductive rubber electrode, $18 \mathrm{~mm}$ diameter) placed on the skin at the midline over the lower thoracic vertebrae. Two $5 \times 9 \mathrm{~cm}$ self-adhesive electrodes (Pro-Patch, Taiwan) were used as anodes, placed bilaterally over the anterior superior iliac spine (Courtine, Harkema et al. 2007, Dy, Gerasimenko et al. 2010, Gorodnichev, Pivovarova et al. 2012). Participants were positioned in supine for the duration of the study.

Surface EMG signals were recorded bilaterally in rectus femoris (RF), vastus lateralis $(\mathrm{VL})$, medial hamstrings $(\mathrm{MH})$ (semitendinosus), tibialis anterior (TA), medial gastrocnemius (MG) lateral soleus (SOL), and flexor hallucis brevis 
(FHB) muscles, using the same electrodes as above. The EMG signals were differentially amplified using MA300 EMG System (Motion Lab Systems, Baton Rouge, LA, USA) with a band-pass filter of $10 \mathrm{~Hz}$ to $2 \mathrm{kHz}(-3 \mathrm{~dB})$. The EMG data were digitized at a sampling rate of $5000 \mathrm{~Hz}$.

The precise location of stimulation for conditioning experiments was determined by the recruitment pattern of MMRs, and standardized such that proximal muscle's thresholds occurred at intensities just below those required to evoke threshold responses in distal muscles (Atkinson et al., 2016, submitted).

\section{Ulnar nerve stimulation}

Ulnar nerve stimulation was applied just proximal to the elbow via bipolar stimulating electrode of fixed inter-electrode distance of $30 \mathrm{~mm}$ (MLADDF30, ADInstruments, New Zealand) using a constant current stimulator (DS5, Digitimer $\AA$, UK). Sensory followed by motor thresholds for the abductor digiti minimi (ADM) were identified, with the presence and latency of the H-reflex confirming the viability of the ulnar nerve after SCI. No participants were recruited with a neurologic level of injury at C8-T1, which provide segmental innervation to the ulnar nerve (Sable 1998). Conditioning stimulation was delivered at $3 x$ motor threshold intensity (Meinck and Piesiur-Strehlow 1981), using three $1 \mathrm{~ms}$ square wave pulses with inter-stimulus interval of $1 \mathrm{~ms}$.

\section{Experimental procedures}

Descending interlimb conditioning of MMRs was investigated as previously described (ref). Briefly, MMR recruitment curves were obtained for identification of optimal test stimulus intensities. Sensory and motor thresholds 
were obtained in $A D Q$ in response to ulnar nerve stimulation, and the conditioning stimulation was set to 3 times motor threshold. Conditioning stimuli were delivered to both left and right ulnar nerves, in separate conditioning bouts randomly ordered within a single experimental session. Condition-test intervals of $40,60,90,110,125,140,160,190,220,250$, and $300 \mathrm{~ms}$ were presented randomly at least 8 times each, along with pre-, interspersed, and post-controls.

\section{Data analysis}

Following $2^{\text {nd }}$ order Butterworth $(40-500 \mathrm{~Hz})$ filtering, EMG from 3 trials of muscle activation were averaged and presented as the ratio of active to baseline EMG (Table 2), with a value of 1.5 or greater indicating an increase in muscle activation during the volitional movement attempt. Participants were grouped based on presence of volitional leg muscle activation: those with motor complete $\mathrm{SCl}$, as identified by a lack of volitional activation of any leg muscle during functional neurophysiological assessment (Li, Atkinson et al. 2012), and those with motor incomplete $\mathrm{SCl}$, who demonstrated repeated, measurable muscle activation in at least one leg muscle.

The magnitude of MMRs was quantified as the peak-to-peak amplitude of the evoked responses. For each conditioning experiment, in each muscle, we fit a generalized additive model (GAM) to the responses as a function of time. The GAM was defined to include a 5-dimensional spline-based smoothing function and a single factor accounting for each CTI tested throughout the experiment. The fitted GAM provided a smoothed trend for the responses over the course of the experiment, so that any changes in unconditioned (control) responses over 
time were accounted by the model. Further, the inclusion of CTI as a factor permitted comparison of mean CTI to control responses for each CTI, with the feature that such comparisons were "localized" to nearby interspersed controls, in order to adequately account for any change in control amplitude or variability during the course of a particular conditioning bout. Standardized conditioning responses were calculated as t-statistics from each GAM model and adjusted per experiment, per muscle using the Benjamini-Hochberg correction with a nominal false discovery rate of 0.05 (Benjamini and Hochberg 1995). A muscle was empirically identified has having been conditioned at a particular CTI if the Benjamini-Hochberg adjusted standardized conditioning response was greater than 2.0. A muscle was defined as having been conditioned overall if it was identified as conditioned at any CTI. The relationship between volitional control and conditioning was evaluated in the RF, VL, MH, TA, and SOL muscles with a chi-square test. All analyses were conducted using the R software environment (R Core Team. (2016). R: A Language and Environment for Statistical Computing. R Foundation for Statistical Computing, Vienna, Austria. V 3.3.1).

\section{$\underline{\text { Results }}$}

FNPA assessment of voluntary muscle activation allowed quantification of active EMG during single joint volitional movement attempts in each participant. Table 2 summarizes the results of volitional movement attempts in 5 bilateral lower limb muscles in which MMR conditioning was also assessed. Seven participants demonstrated volitional muscle activation in at least one leg muscle, while 11 participants were unable to generate active EMG in any leg muscle 
during movement attempts. Based on these results, participants were classified into two groups: motor complete $(n=11)$ or motor incomplete $(n=7)$. Subclinical descending influence was also assessed via reinforcement maneuvers and tonic vibratory reflex. Of the 11 participants who did not demonstrate volitional activation of any leg muscles, positive responses to reinforcement maneuvers were observed in 7 participants, while the presence of tonic vibratory response was identified in 6 (Figure 16). Both signs were present in 4/11 motor complete participants, while neither were present in 2/11. Overall, 9/11 motor complete participants demonstrated some evidence of residual descending influence on spinal motor activity based on a positive response to one of the discomplete criteria (Table 6).

ADM H-reflexes were obtained to confirm viability of the ulnar nerve in all participants. The first peak latency was $28 \pm 4 \mathrm{~ms}$ and $28 \pm 3 \mathrm{~ms}$ for the left and right sides, respectively. In 3 participants, an H-reflex could not be obtained on the left (2) or right (1) side. Conditioning studies were limited to a single ulnar nerve in these cases.

Conditioning stimulation at the ulnar nerve resulted in the facilitation of the test MMR in 59 total muscles (66\%) of $7 / 7$ motor incomplete participants (figure 17), and in 24 total muscles (20\%) of $7 / 11$ motor complete participants (figure 18), demonstrating descending interlimb projections onto lumbosacral motor neuron pools (Table 7). We further subdivided results based on the location of $\mathrm{SCl}$ relative to the segmental innervation of the ulnar nerve (C8), i.e. rostral $(n=10)$ or caudal $(n=8)$ (cf. Table 6, Table 8). Within the motor incomplete group, 
MMR facilitation was observed in $76 \%$ of all muscles in participants with SCI above C8 $(n=3)$, with $59 \%$ of muscles in participants with SCI below C8 $(n=4)$ also demonstrating MMR facilitation (Tables 7, 8). For motor complete participants, 23 of 24 muscles and 6 of 7 participants demonstrating MMR facilitation had $\mathrm{SCl}$ above the $\mathrm{C} 8$ level, with MMR facilitation occurring in just one muscle in 1 of 4 participants with an $\mathrm{SCl}$ below $\mathrm{C} 8$.

For motor incomplete participants, group percentages of the total number of muscles demonstrating MMR facilitation to ipsilateral and contralateral ulnar nerve facilitation, respectively, were as follows: RF: $57 \%$ and $64 \%$, VL: $57 \%$ and 64\%, $\mathrm{MH}: 57 \%$ and $64 \%$, TA: $63 \%$ and $43 \%$, MG: $43 \%$ and $50 \%$, SOL: $43 \%$ and 43\%, and FHB: $60 \%$ and $50 \%$ (Figure 19). For motor complete participants, group percentages of the total number of muscles demonstrating MMR facilitation to ipsilateral and contralateral ulnar nerve facilitation, respectively, were: RF: $14 \%$ and $5 \%$, VL: $9 \%$ and $18 \%, \mathrm{MH}: 14 \%$ and $5 \%$, TA: $9 \%$ and $0 \%$, MG: $14 \%$ and $14 \%$, SOL: $5 \%$ and $5 \%$, and FHB: $5 \%$ and $5 \%$.

As expected, the condition-test intervals at which MMR facilitation occurred varied among participants (Tables 7,8 ). However, in all groups a majority of MMR facilitation occurred at condition-test intervals between $90 \mathrm{~ms}$ and $140 \mathrm{~ms}$.

Volitional activation and interlimb MMR facilitation within a given muscle were found to be related (chi-square test, $\mathrm{P}<.0001)$. MMR facilitation was observed in $72 \%$ of leg muscles (RF, VL, MH, TA, and SOL bilaterally) demonstrating volitional activation (cf. Tables 6, 7). Conversely, MMR facilitation 
was not observed in $83 \%$ of muscles in which no volitional activation was observed. In motor complete participants with a positive reinforcement maneuver, 4/7 demonstrated MMR conditioning, while in those without a positive response, MMR conditioning was observed in 2/4. Similarly, for the tonic vibratory reflex, 4/6 participants with a tonic vibratory reflex also had MMR facilitation, compared to $3 / 5$ motor complete participants without a tonic vibratory response. MMR facilitation was not observed in 2/6 participants demonstrating both discomplete criteria. Among the 7 motor incomplete participants, 3 had a positive reinforcement maneuver, while all 7 had a tonic vibratory reflex.

\section{Discussion}

The current results demonstrate the presence of descending interlimb influence on spinal motor excitability after chronic SCI. Few studies have examined inter-enlargement connectivity after $\mathrm{SCl}$, and such studies have typically applied different methodology and focused primarily on ascending reflexes (Calancie 1991, Calancie, Lutton et al. 1996, Calancie, Alexeeva et al. 2005). One such report recently described modulation of baseline EMG in arm and leg muscles after $\mathrm{SCl}$ in response to superficial peroneal and radial nerve stimulation, respectively. Descending interlimb reflexes in response to radial nerve stimulation were reported in just $6 \%$ (4 total muscles) of ipsilateral and and $0 \%$ contralateral muscles out of 17 participants with cervical SCI (Butler, Godfrey et al. 2016). Considering that Butler et al. used large pulse trains at higher stimulation intensities than applied here, this discrepancy with the current results appears surprising. However, reflex studies quantifying changes in baseline EMG 
typically employ some degree of volitional muscle activation, which improves the likelihood of an evoked response and/or observation of modulation in EMG. Since individuals with motor complete $\mathrm{SCl}$ cannot initiate a volitional contraction below the level of lesion, the current study utilized transcutaneous spinal stimulation to raise the excitability state of the lumbosacral motor circuitry. From this standpoint, the current results are in agreement with recent studies of both transcutaneous and epidural stimulation, which have demonstrated that the excitability state of the lumbosacral circuitry may be modulated by electrical stimulation, allowing the integration of peripheral afferents and descending inputs, and resulting in the activation of paralyzed muscles (Angeli, Edgerton et al. 2014, Gerasimenko, Lu et al. 2015, Rejc, Angeli et al. 2015).

\section{Pathways mediating interlimb MMR modulation}

The current study included individuals with SCI both above and below the segmental innervation of the ulnar nerve at C8 (Kendall, McCreary et al. 1983, Sable 1998), in order to gain insight on the nature and prevalence of descending interlimb reflexes in individuals with injuries proximal to the proposed interenlargement circuitry being studied ( $\mathrm{SCl}$ above $\mathrm{C} 8)$, as well as those with an injury between the cervical and lumbosacral enlargements ( $\mathrm{SCl}$ below $\mathrm{C} 8)$. As expected based on differences in mechanism, spinal level, and severity of injury among participants, results varied considerably among participants and within both the motor complete and the motor incomplete groups (Table 7). When taking into account both level of injury and injury severity, it was observed that MMR facilitation only occurred in a single muscle in one of four participants with 
motor complete injury in the thoracic segments (Table 8). Conversely, descending interlimb reflexes were observed in 6 of 7 motor complete participants injured rostral to $\mathrm{C} 8$, indicating that sparing and/or re-establishment of descending interlimb reflexes occurs more frequently in individuals with more rostral cervical injuries.

Descending interlimb facilitation studies in neurologically intact participants suggest that multiple reflex pathways may be involved in the observed MMR modulation, with propriospinally mediated facilitation at early latencies, and supraspinally mediated facilitation occurring at longer latencies (submitted) (Meinck and Piesiur-Strehlow 1981, Zehr, Collins et al. 2001). Our studies of vestibulospinal (in review) and reticulospinal (manuscript in preparation) further support this interpretation, as do the current results. Similarly, others investigating the reflex effects of these pathways on the soleus H-reflex via galvanic vestibular stimulation (Kennedy and Inglis 2001) or acoustic startle (Rossignol and Jones 1976) found the strongest facilitation at 100ms and 110-130ms, respectively. When comparing the latencies at which MMR facilitation was first detected, facilitation at early latencies $(60-90 \mathrm{~ms})$ represented $18 \%$ of the total number of observations in motor incomplete participants, while long latencies (140ms-300ms) represented $59 \%$. In contrast, in motor complete participants, facilitation at early latencies represented $40 \%$ of all occurrences of MMR facilitation, with long latencies representing $37 \%$. The relative increase in early latency facilitation and decrease in long latency facilitation in motor complete individuals may represent the expected sparing of inter-enlargement 
projections located caudal to the level of lesion, while the substantial loss of supraspinal input in these more severely injured participants is represented both by the decrease in the total number of reflex observations, and by the relative decrease in MMR facilitation at longer latencies (Table 8).

\section{MMR facilitation and volitional activation within lumbosacral motor pools}

Long descending propriospinal neurons within the cervical enlargement have been shown to receive excitatory input from both reticulospinal and corticospinal tract fibers in mice, rats, cats, and primates (Alstermark, Lundberg et al. 1987, Rosenzweig, Courtine et al. 2010, Ni, Nawabi et al. 2014, Mitchell, McCallum et al. 2016) possibly providing an alternative route for supraspinal input which would allow integration of relevant afferent information 'en route' to the lumbosacral motor networks. Further, following incomplete $\mathrm{SCl}$ in animal models, propriospinal neurons have been shown to play a role in re-establishing supraspinal influence on spinal circuits caudal to the injury (Bareyre, Kerschensteiner et al. 2004, Courtine, Song et al. 2008, Filli, Engmann et al. 2014), accomplished by the sprouting of severed cortico- and reticulospinal axons to establish synaptic contacts with propriospinal neurons, which then relay the descending signal around the zone of injury. The current study examined the relationship between descending interlimb pathways and volitional muscle activation below the level of lesion. While volitional activation of a muscle and presence of MMR modulation was found to be related $(p<.0001)$, it should be noted that this relationship varied among participants. Interlimb modulation was observed in just a single muscle in two participants who demonstrated volitional 
activation of $9 / 10$ and $10 / 10$ leg muscles tested and were community ambulators functionally. In contrast, in two other participants who demonstrated similar volitional activation (9/10 and 10/10 of tested muscles) and were also community ambulators, MMR modulation was observed in all but a single muscle (Tables 6, 7). Additionally, one participant demonstrated voluntary activity in knee, ankle, and foot muscles of the left leg only, however MMR facilitation was observed bilaterally in response to both left and right ulnar nerve stimulation, but was primarily confined to knee muscles representing the most proximal lumbar segments (Figure 20). This variability among participants is difficult to interpret in the absence of information regarding the viability of other major descending projections, but suggests that relative sparing of different descending systems can lead to similar functional recovery.

In the current study, the presence of either a positive reinforcement maneuver or of a tonic vibratory reflex in sublesional muscles did not predict the presence of descending interlimb MMR modulation. Positive responses to these maneuvers have been purported to indicate an anatomically and physiologically incomplete $\mathrm{SCl}$ in the absence of motor or sensory function below the level of lesion, but are not correlated to motor and/or sensory function (Sherwood, Dimitrijevic et al. 1992). Although it was suggested that they are most frequently seen in individuals with spastic hypertonia, we did not assess this relationship in the current study. To the author's knowledge, no correlation with any diagnostic measures or clinical assessments has been found, which previously raised 
speculation that the spread of muscle activation observed may be mechanical rather than neural(Sherwood, Dimitrijevic et al. 1992).

\section{$\underline{\text { Conclusions }}$}

The current study revealed the existence of descending interlimb projections onto the lumbosacral motor excitability in SCI participants with a spectrum of injury severities sustained at various rostro-caudal levels, including those with motor complete injuries. Differences were observed in the nature and extent of descending modulatory inputs, both across and within clinically defined injury categories, which cannot be appreciated using currently available clinical methods of injury classification. These results reflect the anatomical and physiological heterogeneity which exists in clinical SCI populations, and suggest that assessment of descending spinal transmission as reported here may be used to identify and better account for these differences among study participants, which has direct implications on clinical diagnosis, prognosis, and intervention prescription, as well as for researchers investigating recovery of motor function over time and/or as a result of therapeutic intervention. Future studies incorporating additional stimulation paradigms and comprehensive assessment of the major descending pathways will be important to further our understanding of the mechanisms allowing the emergence of volitional muscle activitation/modulation observed in the presence of spinal electrical stimulation. 
Table 5. Patient Demographics

\begin{tabular}{|c|c|c|c|c|c|c|c|}
\hline Participant & gender & $\begin{array}{l}\text { Age } \\
\text { (yrs.) }\end{array}$ & $\begin{array}{l}\text { time post } \\
\text { injury (yrs.) }\end{array}$ & $\begin{array}{c}\text { AIS } \\
\text { Neuro } \\
\text { Level }\end{array}$ & $\begin{array}{c}\text { AlS } \\
\text { Class. }\end{array}$ & Mechanism Of Injury & $\begin{array}{l}\text { Primary } \\
\text { Mobility }\end{array}$ \\
\hline A37 & $\mathrm{m}$ & 29 & 6.11 & T2 & A & motorcycle accident & Manual w/c \\
\hline A41 & $\mathrm{m}$ & 24 & 6.80 & C4 & A & skiing accident & Manual w/c \\
\hline A59 & $\mathrm{m}$ & 26 & 2.32 & $\mathrm{~T} 4$ & A & motor vehicle accident & Manual w/c \\
\hline A60 & $\mathrm{m}$ & 22 & 2.28 & T4 & $A$ & motorcycle accident & Manual w/c \\
\hline A68 & $\mathrm{m}$ & 34 & 3.47 & C5 & A & assault victim & Power w/c \\
\hline $\mathrm{B} 19$ & $\mathrm{~m}$ & 41 & 1.03 & $\mathrm{C} 6$ & $B$ & motor vehicle accident & Power w/c \\
\hline $\mathrm{B} 20$ & $\mathrm{~m}$ & 28 & 4.18 & $\mathrm{~T} 2$ & $\mathrm{~B}$ & fall & Manual w/c \\
\hline B21 & $\mathrm{m}$ & 29 & 5.15 & C4 & $B$ & motor vehicle accident & Manual w/c \\
\hline $\mathrm{B} 23$ & $\mathrm{~m}$ & 32 & 2.97 & $\mathrm{C} 5$ & $\mathrm{~B}$ & mountain bike accident & Manual w/c \\
\hline $\mathrm{C} 21$ & $f$ & 24 & 1.92 & $\mathrm{C} 4$ & C & hit by trolley & Power w/c \\
\hline $\mathrm{C} 32$ & $\mathrm{~m}$ & 35 & 5.32 & $\mathrm{~T} 2$ & $\mathrm{C}$ & motorcycle accident & Manual w/c \\
\hline C34 & $\mathrm{m}$ & 20 & 4.50 & $\mathrm{C} 4$ & C & hockey accident & Power w/c \\
\hline $\mathrm{C} 36$ & $\mathrm{~m}$ & 24 & 1.35 & T6 & $\mathrm{C}$ & motor vehicle accident & Manual w/c \\
\hline $\mathrm{C} 35$ & $\mathrm{~m}$ & 21 & 1.58 & $\mathrm{C7}$ & $\mathrm{D}$ & diving Accident & Manual w/c \\
\hline $\mathrm{D} 36$ & $\mathrm{~m}$ & 26 & 9.53 & $\mathrm{C} 5$ & $D$ & football accident & Amb w/o AD \\
\hline D37 & $\mathrm{m}$ & 28 & 6.72 & T4 & D & $\begin{array}{c}\text { spontaneous epidural } \\
\text { hematoma }\end{array}$ & Amb w/o AD \\
\hline D38 & $\mathrm{m}$ & 42 & 12.99 & $\mathrm{C} 2$ & D & hit by falling object & Amb w/SPC \\
\hline D41 & $f$ & 44 & 5.41 & T4 & D & motor vehicle accident & Amb w/o AD \\
\hline
\end{tabular}


Table 6. FNPA results summary

\begin{tabular}{|c|c|c|c|c|c|c|c|c|c|c|c|c|}
\hline \multirow{2}{*}{ Participant } & \multicolumn{2}{|c|}{ RF } & \multicolumn{2}{|c|}{ VL } & \multicolumn{2}{|c|}{$\mathrm{MH}$} & \multicolumn{2}{|c|}{ TA } & \multicolumn{3}{|c|}{ SOL } & \multirow[b]{2}{*}{ T.V.R. } \\
\hline & eft & Right & Left & Right & Left & Right & Left & Right & Left & Righ & .M & \\
\hline C36 & 3.5 & 5.2 & 13.5 & 3.9 & 33.5 & 10.4 & 1.9 & 0.0 & 9.7 & 1.2 & + & present \\
\hline D38 & 7.5 & 15.3 & 20.5 & 10.8 & 1.0 & 15.7 & 3.4 & 29.2 & 10.0 & 2.0 & - & present \\
\hline D37 & 70.0 & 23.5 & 10.5 & 30.3 & 34.1 & 29.3 & 58.2 & 50.6 & 10.2 & 9.4 & + & present \\
\hline D36 & 58.2 & 55.6 & 17.2 & 31.0 & 25.2 & 43.0 & 74.5 & 139.7 & 39.9 & 23.5 & - & present \\
\hline C32 & 16.3 & 0.0 & 18.3 & 0.1 & 13.0 & 0.0 & 12.7 & 0.0 & 1.9 & 0.0 & - & present \\
\hline D41 & 88.9 & 87.6 & 36.1 & 50.6 & 20.6 & 15.6 & 95.2 & 31.5 & 14.1 & 14.8 & + & present \\
\hline C35 & 9.3 & 26.3 & 0.0 & 0.1 & 3.8 & 0.1 & 36.8 & 0.1 & 6.6 & 0.1 & + & present \\
\hline A37 & 0.0 & 0.1 & 0.0 & -0.1 & 0.1 & 0.0 & -0.1 & -0.2 & 0.1 & 0.0 & + & absent \\
\hline A41 & 0.0 & 0.0 & 0.0 & 0.1 & 0.0 & 0.0 & 0.0 & 0.0 & 0.0 & 0.0 & + & absent \\
\hline B19 & 0.0 & 0.0 & 0.1 & 0.0 & 0.2 & 0.0 & 0.0 & 0.0 & 0.0 & 0.0 & + & present \\
\hline B21 & 0.0 & 0.1 & 0.0 & 0.0 & 0.0 & 0.0 & -0.1 & 0.0 & 0.0 & 0.0 & + & present \\
\hline B20 & 0.0 & 0.0 & 0.0 & 0.1 & 0.0 & 0.0 & -0.1 & 0.0 & 0.0 & 0.0 & + & present \\
\hline C21 & 0.0 & 0.1 & 0.0 & 0.1 & 0.0 & 0.0 & 0.0 & 0.0 & 0.0 & 0.0 & + & present \\
\hline A59 & 0.0 & 0.0 & 0.0 & 0.0 & 0.0 & 0.1 & -0.1 & 0.0 & -0.1 & 0.0 & + & absent \\
\hline B23 & 0.0 & 0.0 & 0.0 & 0.0 & 0.0 & 0.0 & 0.0 & 0.0 & 0.0 & 0.0 & - & present \\
\hline A68 & 0.0 & 0.0 & 0.0 & 0.0 & 0.0 & 0.0 & 0.0 & 0.0 & 0.0 & 0.1 & - & absent \\
\hline C34 & 0.0 & 0.0 & 0.0 & 0.0 & 0.0 & 0.0 & -0.1 & 0.0 & 0.0 & 0.0 & - & present \\
\hline A60 & 0.1 & 0.0 & 0.0 & 0.0 & 0.0 & 0.0 & 0.0 & 0.0 & 0.0 & 0.0 & - & absent \\
\hline
\end{tabular}

Volitional movement attempts for bilateral RF, VL, MH, TA, and SOL quantified as the ratio of active to baseline EMG (avg. of 3 attempts). Bold indicates volitional activation. Results of reinforcement maneuvers and tonic vibratory reflex are indicated as positive or negative. 
Figure 16. Positive reinforcement maneuver and tonic vibratory reflex
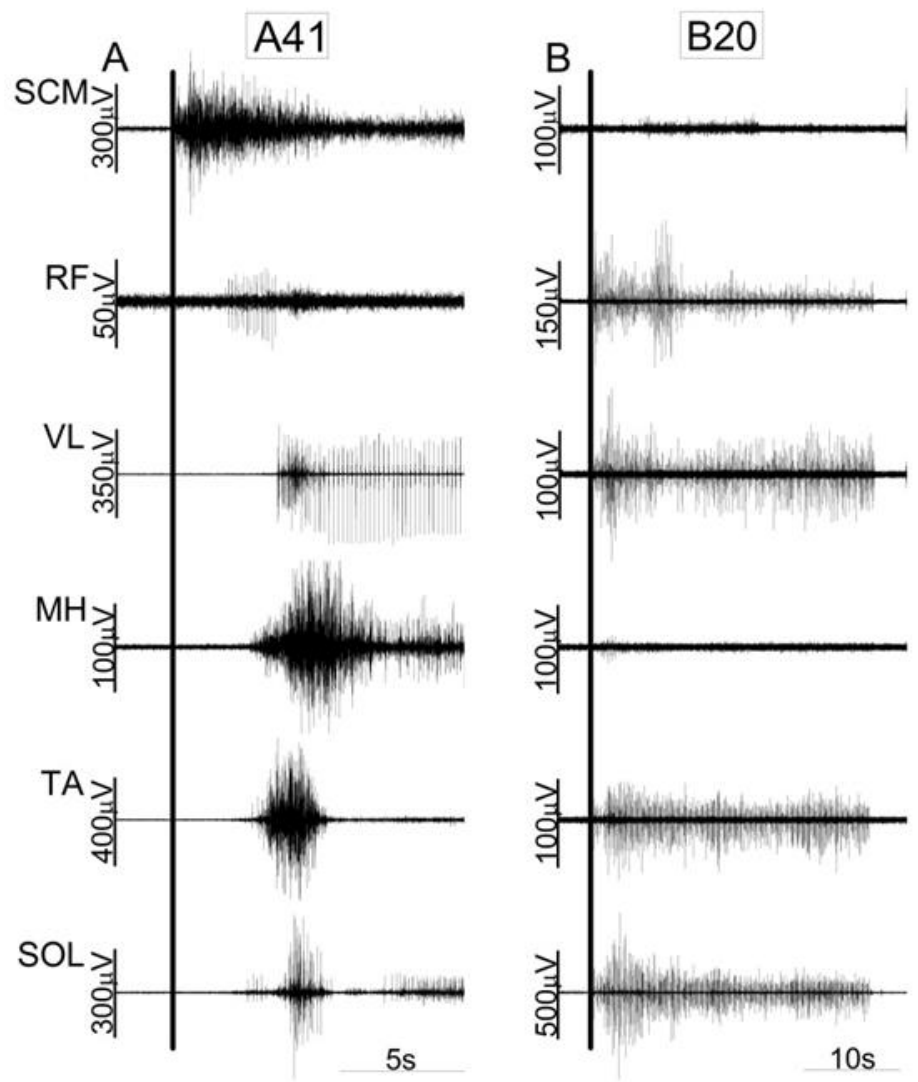

Panel A depicts an example of a positive response to reinforcement maneuver, while panel $B$ depicts an example of a tonic vibratory reflex, in representative participants. EMG from SCM, RF, VL, MH, TA, and MG muscles is presented. The vertical black lines represent the onset of the respective trials. For tonic vibratory reflex, SCM and knee muscles' (RF, VL, MH) EMG taken from patellar tendon vibration trial, and ankle muscles' (TA and SOL) EMG taken from Achilles tendon vibration trial. 
Table 7. MMR facilitation latency by participant and muscle

\begin{tabular}{|c|c|c|c|c|c|c|c|c|c|c|c|c|c|c|c|}
\hline ID & $\begin{array}{c}\text { Injury } \\
\text { level }\end{array}$ & LRF & RRF & LVL & RVL & LMH & RMH & LTA & RTA & LMG & RMG & LSOL & RSOL & LAHB & RAHB \\
\hline C35 & C7 & $90(110)$ & $90(110)$ & $90(110)$ & $90(110)$ & $90(110)$ & $90(110)$ & 90 & $90(110)$ & $90(110)$ & $90(110)$ & 110 & 110 & 110 & 110 \\
\hline D36 & C5 & $90(140)$ & $90(140)$ & $90(140)$ & $90(110)$ & $110(140)$ & $90(190)$ & $90(140)$ & $90(190)$ & $90(140)$ & $110(190)$ & $90(140)$ & $90(140)$ & $90(140)$ & $90(140)$ \\
\hline D38 & $\mathrm{C} 2$ & & & & & 90 & & & & & & & & & \\
\hline A41 & $\mathrm{C} 4$ & & $90(110)$ & & 90 & & & & & & & & & & \\
\hline B21 & $\mathrm{C} 4$ & 90 & 90 & 90 & $60(90)$ & & & & $90(110)$ & & $60(140)$ & & & & \\
\hline B23 & C5 & $90(190)$ & & 90 & 250 & 110 & & & & $60(110)$ & $60(110)$ & $60(110)$ & & $60(90)$ & $60(110)$ \\
\hline B19 & $\mathrm{C} 6$ & & & & & & & & & & & & & & \\
\hline $\mathrm{C} 21$ & $\mathrm{C} 4$ & & & & & & & & & 110 & 300 & & & & \\
\hline A68 & $\mathrm{C} 5$ & & & & & & & & & & 110 & & & & 90 \\
\hline C34 & $\mathrm{C} 4$ & & & 90 & & 90 & & & & & & & & & \\
\hline D41 & $\mathrm{T} 4$ & $110(140)$ & $110(190)$ & $90(190)$ & 110 & $110(190)$ & $90(190)$ & $140(190)$ & $90(140)$ & $140(300)$ & $90(140)$ & & $90(190)$ & $110(140)$ & $90(190)$ \\
\hline C32 & $\mathrm{T} 2$ & $90(110)$ & 90 & 90 & 90 & & & & & & & 90 & $90(190)$ & & \\
\hline C36 & T6 & 90 & 90 & $60(90)$ & $60(90)$ & $90(140)$ & $60(110)$ & $90(110)$ & 110 & $90(110)$ & & 110 & & & \\
\hline D37 & $\mathrm{T} 4$ & & & & & & & & & & & & 110 & & \\
\hline A59 & $\mathrm{T} 4$ & & & & & & & & & & & & 110 & & \\
\hline $\mathrm{A} 60$ & T4 & & & & & & & & & & & & & & \\
\hline A37 & $\mathrm{T} 2$ & & & & & & & & & & & & & & \\
\hline B20 & $\mathrm{T} 2$ & & & & & & & & & & & & & & \\
\hline
\end{tabular}

For each participant and muscle, the condition-test interval (CTI) at which MMR facilitation reached statistical significance is listed, with the CTI at which peak facilitation was observed (if different) given in parenthesis.

Participants in gray are motor incomplete participants, and the horizontal black line separates participants with injuries above and below the C8 segmental level. 
Figure 17. Volitional activation and MMR conditioning in a motor incomplete participant

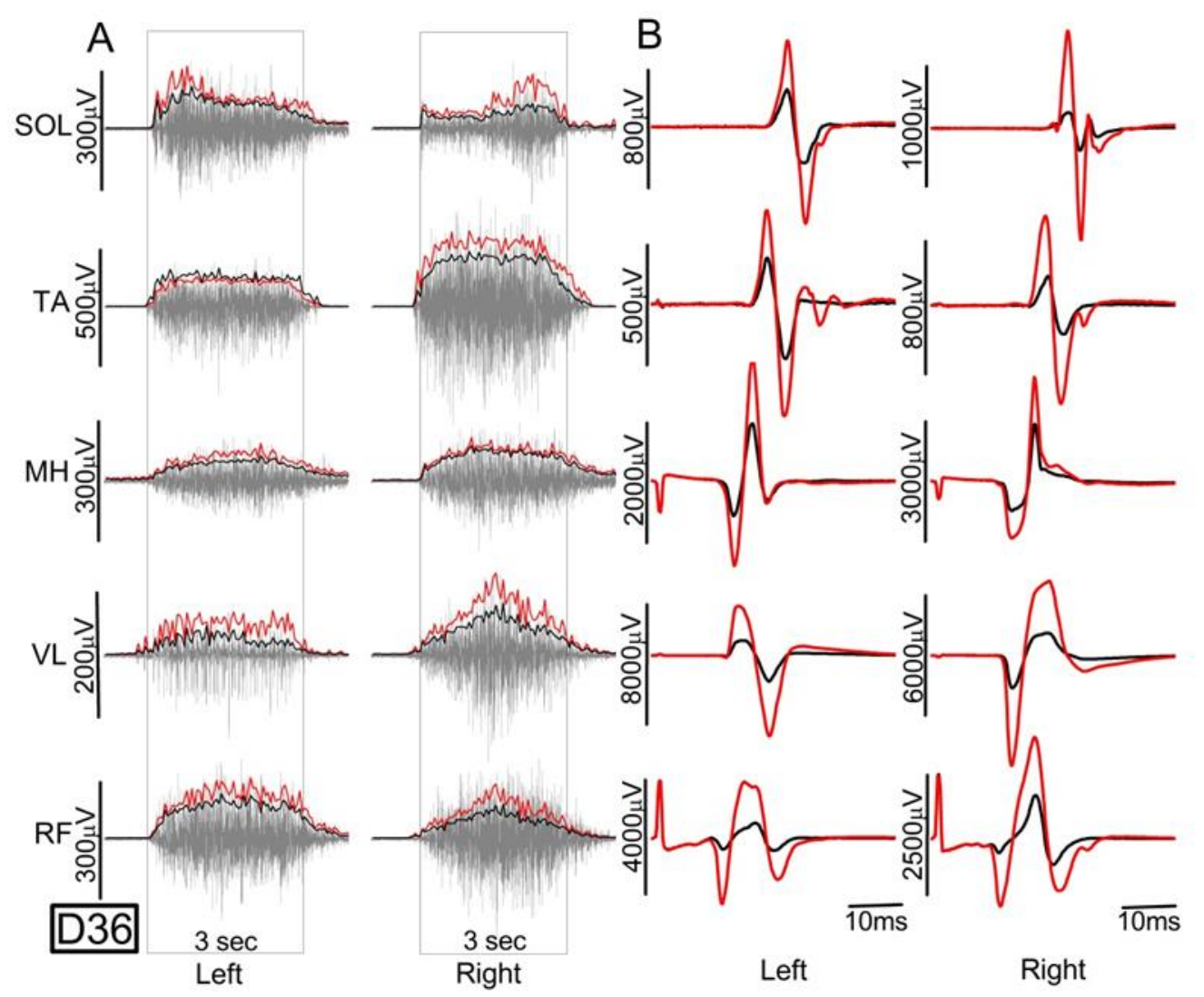

Panel A depicts averaged EMG from RF, VL, MH, TA, and SOL during 3 volitional activation attempts for each muscle. The black trace represents the average EMG envelope, and the red line indicates one standard deviation. The box indicates the 3 second period during which the participant attempted to activate the muscle. Panel B depicts the results of MMR conditioning via right ulnar nerve stimulation in the same muscles. Black traces are the average of 8 control MMRs, red traces are the average of 8 conditioned MMRs (condition test interval $=140 \mathrm{~ms})$. 
Figure 18. Volitional activation and MMR conditioning in motor complete participants

B21

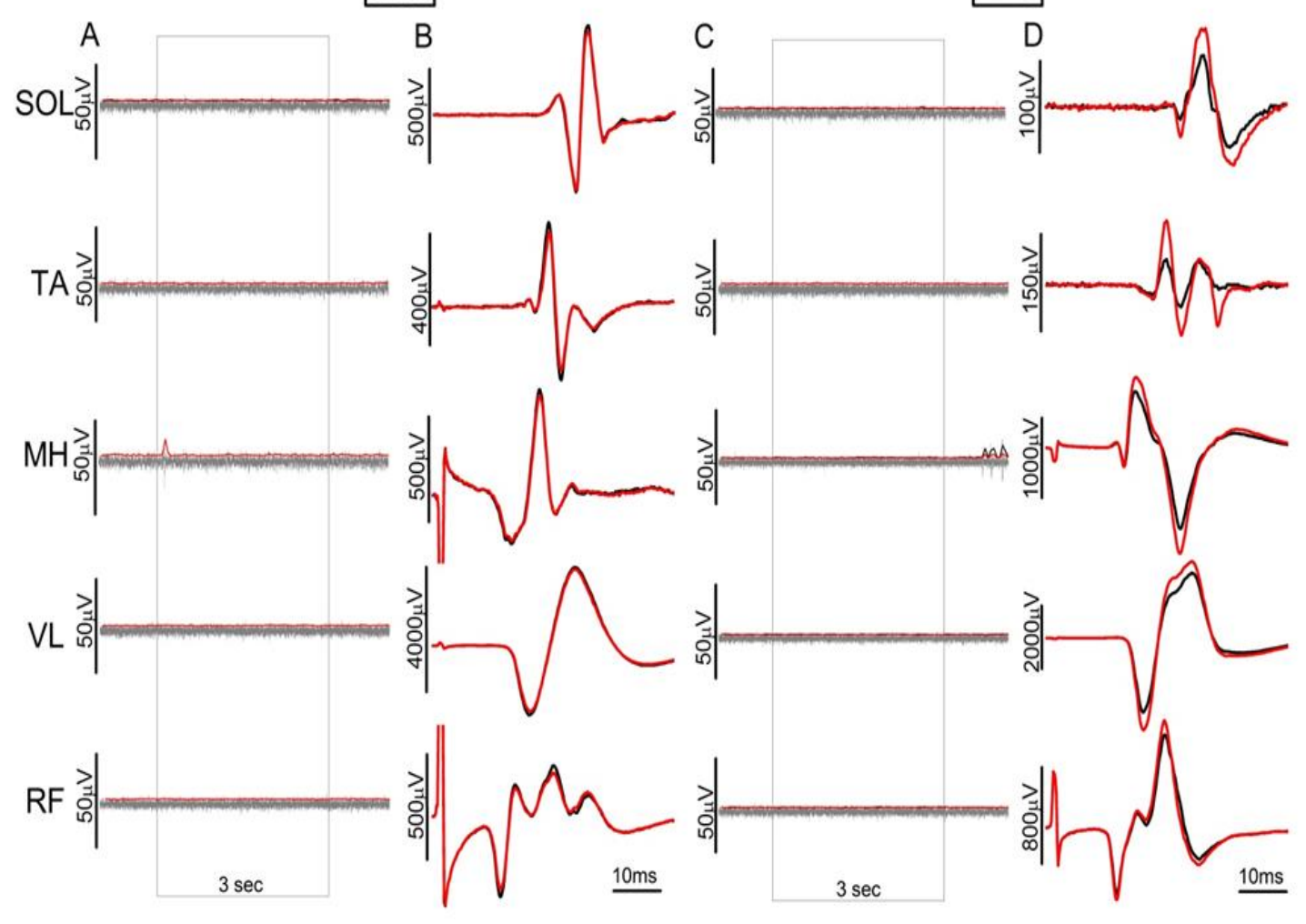

Two participant examples are shown, with panels $A$ and $C$ depicting averaged EMG from right $\mathrm{RF}, \mathrm{VL}, \mathrm{MH}, \mathrm{TA}$, and $\mathrm{SOL}$ during 3 volitional activation attempts for each muscle. The black trace represents the average EMG envelope, and the red line indicates one standard deviation. The box indicates the 3 second period during which the participant attempted to activate the muscle. Panels $B$ and $D$ depict the results of MMR conditioning experiments in the same muscles. Black traces are the average of 8 control MMRs, red traces are the average of 8 conditioned MMRs at a condition-test interval of $110 \mathrm{~ms}$. 
Figure 19. Group MMR conditioning results

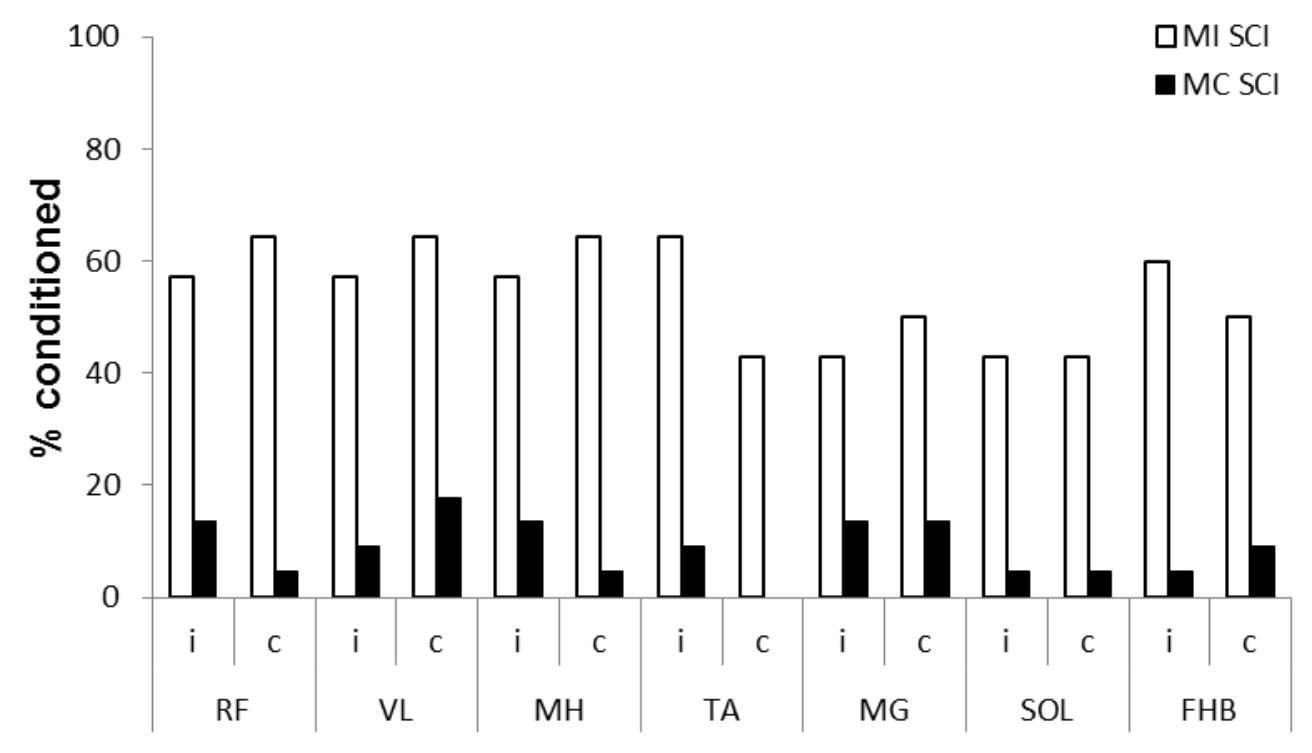

Percentage of muscles demonstrating MMR facilitation at any condition-test interval are illustrated for motor incomplete (MI) and motor complete (MC) participants in response to both ipsilateral (i) and contralateral (c) ulnar nerve stimulation. 
Table 8. Summary of MMR facilitation by group and latency.

\begin{tabular}{|c|c|c|c|c|c|}
\hline \multirow{3}{*}{ CTI } & \multicolumn{4}{c|}{ MI } \\
\cline { 2 - 6 } & $\begin{array}{c}\text { Above } \\
\text { C8 }\end{array}$ & $\begin{array}{c}\text { Below } \\
\text { C8 }\end{array}$ & $\begin{array}{c}\text { Above } \\
\text { C8 }\end{array}$ & $\begin{array}{c}\text { Below } \\
\text { C8 }\end{array}$ & Total \\
\hline 60 & $2(2)$ & $4(2)$ & $8(3)$ & $1(1)$ & $15(8)$ \\
90 & $29(3)$ & $23(4)$ & $16(5)$ & $1(1)$ & $69(13)$ \\
110 & $45(2)$ & $32(4)$ & $14(6)$ & $1(1)$ & $92(13)$ \\
140 & $46(2)$ & $30(4)$ & $5(4)$ & $1(1)$ & $82(11)$ \\
190 & $32(2)$ & $25(4)$ & $6(3)$ & $1(1)$ & $64(10)$ \\
250 & $14(1)$ & $19(2)$ & $5(3)$ & $1(1)$ & $39(7)$ \\
300 & $13(1)$ & $18(1)$ & $6(4)$ & $1(1)$ & $38(7)$ \\
\hline Total & $76 \%(3)$ & $59 \%(4)$ & $30 \%(6)$ & $2 \%(1)$ \\
\hline
\end{tabular}

Number of observations of MMR facilitation in any muscle for each group and condition-test interval $(\mathrm{CTI})$ are listed, with the corresponding number of participants in parenthesis. The far right column gives the total number of MMR facilitation observations at each CTI, while the bottom totals give the percentage of muscles in which MMR facilitation was observed for each group. 
Figure 20. MMR facilitation in a participant with unilateral volitional activation.
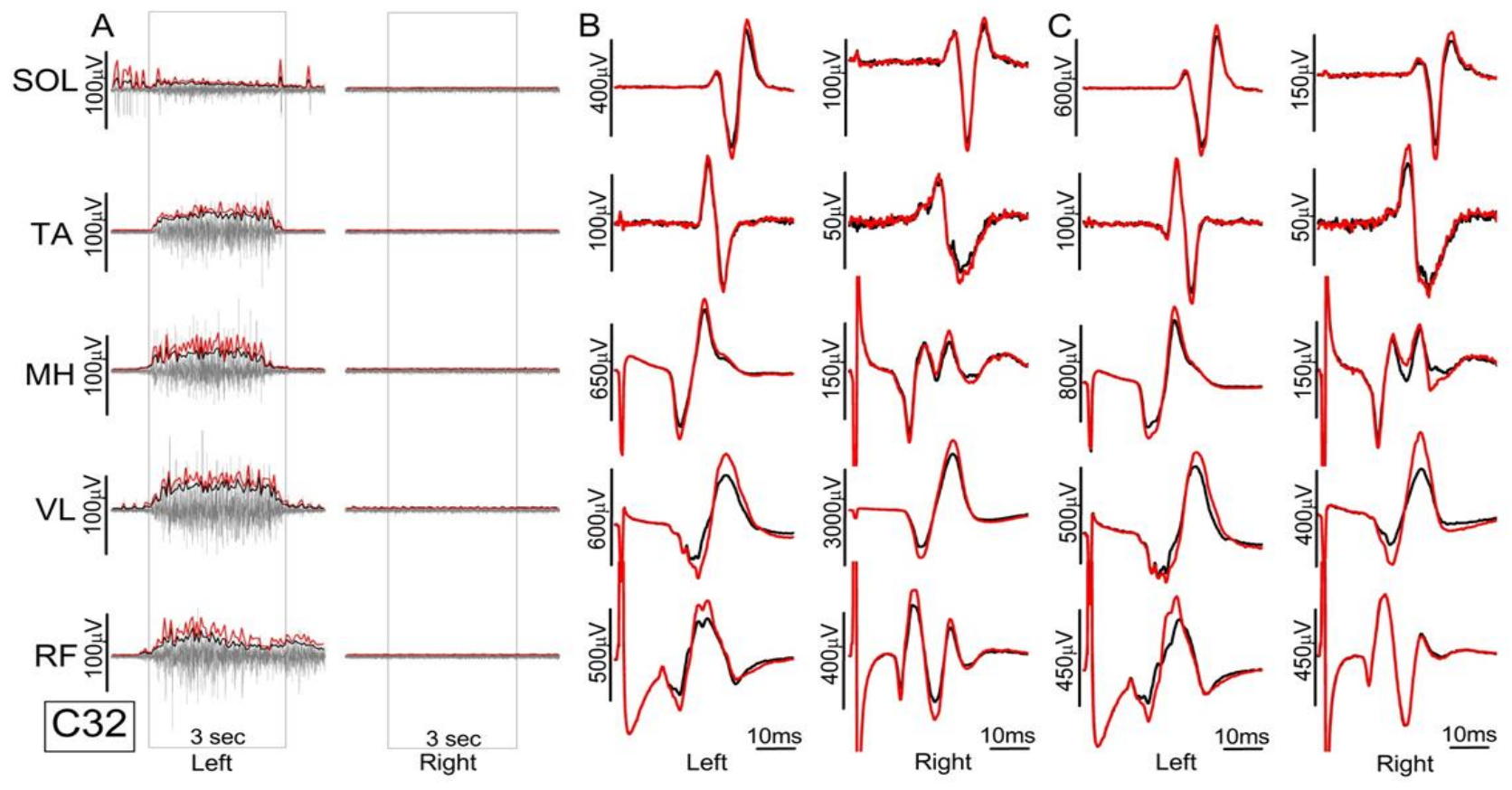

Panel A depicts averaged EMG from RF, VL, MH, TA, and SOL during 3 volitional activation attempts for each muscle. The black trace represents the average EMG envelope, and the red line indicates one standard deviation. The gray box indicates the 3 second period during which the participant attempted to activate the muscle. Panels $B$ and $C$ depict the results of MMR conditioning via left and right ulnar nerve stimulation, in the same muscles. Black traces are the average of 8 control MMRs, red traces are the average of 8 conditioned responses at a conditiontest interval of $110 \mathrm{~ms}$. 


\section{CHAPTER V}

\section{DISCUSSION}

This project aimed to assess the residual and/or de novo descending interlimb influence on lumbosacral motor neuron excitability after clinically complete spinal cord injury, and determine the correlation between electrophysiological identification of descending spinal transmission and neurophysiological measurements of volitional, sublesional muscle activation after incomplete spinal cord injury. This work is part of a broader project, which to our knowledge represents the first attempt to systematically investigate the nature and extent of descending projections of the major descending tracts onto the lumbosacral motor circuitry. Previous studies have suggested that residual supraspinal-spinal connections may exist in individuals clinically classified as having complete spinal lesions (Sherwood, Dimitrijevic et al. 1992, Kakulas and Kaelan 2015), but thus far few attempts have been made to determine the whether the pathways mediating such connections can be identified, and nearly all of these focused solely on the corticospinal tract (Curt, Keck et al. 1998, Calancie, Alexeeva et al. 1999, McKay, Lee et al. 2005) or ascending interlimb reflexes (Calancie 1991, Calancie, Lutton et al. 1996). Although propriospinal pathways have been investigated following human $\mathrm{SCl}$ previously, this work focused on ascending pathways (Calancie, Alexeeva et al. 2005), concluding that 
the appearance and strengthening over time of ascending interlimb reflexes represents maladaptive plasticity and is suggestive of a functionally complete spinal lesion. Here, descending interlimb reflexes were investigated to assess the propriospinal system, based in part on evidence from multiple animal models that propriospinal fibers participate in the restoration of functional cortical input to the sub-lesional spinal circuitry (Bareyre, Kerschensteiner et al. 2004, van den Brand, Heutschi et al. 2012, Filli, Engmann et al. 2014). In order to assess whether descending propriospinal projections might play a role in the recovery of volitional movement after human $\mathrm{SCl}$, we also developed methods for the quantitative assessment of voluntary activation of the leg muscles.

A standardized, objective assessment of volitional motor capacity, the Functional Neurophysiological Assessment (FNPA) was developed (Li, Atkinson et al. 2012), which expands upon the previously validated Brain Motor Control Assessment (Sherwood, McKay et al. 1996). The FNPA provides increased sensitivity in the detection of both volitional and reflex muscle activation by virtue of the standardized nature of the assessment, as well as the objective and quantitative analysis methods. One advantage of this method is the ability to measure muscle activation in muscles innervated by the thoracic segments, which are currently not assessed by the AIS exam. While the standard clinical neurological examination assesses sensory function at all dermatomes, motor function is typically only assessed in limb muscles innervated by the cervical and lumbosacral segmental levels. This means that motor function throughout the entirety of the thoracic spine is not assessed. Unlike the appendicular 
musculature, whose peripheral innervation arises from a neural plexus and contains axons from multiple segmental levels, the axial musculature is innervated by anterior and posterior rami from each segmental level. In addition, these muscles have differing functional roles, with axial musculature being principally involved in breathing, posture, and proximal stabilization during movement of the extremities- all of which are typically not under voluntary control- in addition to volitional movements. Further, while dermatomes and myotomes are thought to occupy the same territory in the thoracic region, this is not always the case, with the most well-known example being the diaphragm. Another important advantage is the ability to assess multi-muscle patterns of activation, which are altered after $\mathrm{SCl}$, and may change over time, coincident with movement recovery (McKay, Ovechkin et al. 2011, Li, Atkinson et al. 2012). As volitional movement recovers, multi-muscle EMG patterns may be initially characterized as widespread muscle activation with a high degree of agonstantagonist co-contraction. As recovery progresses further, the overall magnitude of EMG activity decreases, becoming isolated to the muscles required for the desired movement, with a decrease in co-activation (McKay, Ovechkin et al. 2011). Interestingly, this seemingly staged process of skilled movement recovery mirrors novel skill acquisition and the development of synergies in the noninjured nervous system (Asaka, Wang et al. 2008).

The objectively quantifiable nature of the FNPA presents clear advantages to subjective assessment of motor function as is the current clinical standard. The main advantages involve the number of muscles studied, in particular the 
inclusion of axial musculature. more than 150 FNPA studies were conducted on SCI participants, including those presented here, and greater than $90 \%$ demonstrated volitional activation of erector spinae muscles recorded at T10, regardless of severity, and was only absent and in subset of high cervical injuries. In contrast, the rectus abdominus was volitionally active in less than $10 \%$ of motor complete injuries rostral to T6.

Of note, the axial extensors have a distinctly different innervation pattern than axial flexors and appendicular muscles, with axial extensors being innervated by the posterior rami of the ventral root, while anterior axial and appendicular muscles are innervated by the anterior rami(Shinoda, Sugiuchi et al. 2006). In terms of excitability, the axial extensor motor pools are more strongly innervated by brainstem tracts responsible for balance and posture, in comparison with direct corticospinal inputs. The reverse is true for appendicular muscles, with an apparent gradient from proximal to distal muscles (Shinoda, Sugiuchi et al. 2006).

The relative involvement of cortical and brainstem tracts in volitional activation in these muscles is unclear. It was asserted that corticospinal inputs played a significant role based on transcranial stimulation(TMS) induced motor evoked potentials, however there are multiple problems with the interpretation of these results (Jaberzadeh, Zoghi et al. 2013). First, TMS is frequently used to measure 'corticospinal excitability'(Rossini, Burke et al. 2015) however, there is no direct measurement of corticospinal activity. As the only measurable variable is the amplitude of motor responses, any change in the excitability of spinal motor 
neurons (whether induced by spinal, corticospinal, or other descending tract mechanisms) could lead to the same result. Much focus has been put on the latencies as evidence of a direct cortico-motoneuronal route of transmission, however it is well known that latencies are decreased by as much as $3 \mathrm{~ms}$ when volitional activation of the studied muscle is performed during TMS (Rossini, Burke et al. 2015). Speculation involving excitation of corticospinal axons of different sizes predominates over any suggestion that additional synapses may be involved in motor evoked potentials evoked from near threshold TMS intensities, although the latency discrepancy is clearly long enough for 2-3 synapses. As it relates to the mentioned study of erector spinae muscles, this group found latencies in motor evoked potentials recorded supra-umbilically ( T10 vertebral level) in the rectus abdominus occurred approximately $3 \mathrm{~ms}$ sooner than those recorded at the erector spinae at L3-L4 (Jaberzadeh, Zoghi et al. 2013). Thus the results in this particular study, and in TMS studies in general, are far from conclusive regarding the mechanisms of volitional muscle activation.

Regardless of the mechanisms involved, the extent of axial muscle sparing and potential recovery following high-thoracic and cervical $\mathrm{SCl}$ has been investigated little, likely due to the lack of available measures, namely the lack of inclusion on axial muscles in the International Standards for Neurological Classification of Spinal Cord Injury (Kirshblum, Waring et al. 2011). Unpublished clinical evidence suggests that task-specific interventions aimed at re-training trunk control after spinal cord injury can improve posture and balance after motor complete SCl. Suggesting the apparent sparing of spinal extensor function 
following many injuries, as observed in the FNPA, may have functional relevance. Unfortunately, current clinical SCI rehabilitation textbooks teach that high thoracic and cervical motor complete SCl individuals cannot regain trunk control due to paralysis of these muscles, leading to early training using compensatory techniques, and a lack task-specific, non-compensatory balance and posture training.

The FNPA addresses the need for measurement tools assessing the axial musculature after $\mathrm{SCl}$, along with the other mentioned advantages. The FNPA is feasible to perform clinically as early after injury as the inpatient rehabilitation setting. Objective monitoring of motor function throughout the acute and subacute period after SCl may improve diagnosis and prognosis, and give clinicians new insights on the available residual motor system and its functional implications. This could, in turn, directly impact intervention selection and therapeutic decision making.

\section{Descending Interlimb Modulation of MMRs in neurologically intact participants}

Novel electrophysiological methods for the detection of descending propriospinal input on lumbosacral motor neuron excitability were also developed, and normative data collected. A condition-test paradigm was designed, utilizing transcutaneous spinal electrical stimulation to evoke MMRs in bilateral knee and ankle muscles representing all lumbosacral segments. Robust descending interlimb facilitation of bilateral knee and ankle muscles could be routinely observed in non-injured subjects, with early latencies ( $\leq 90 \mathrm{~ms})$ 
consistent with descending propriospinal transmission. As expected, the overall time-course and magnitude of the effects was in agreement with similar studies investigating descending interlimb modulation of the soleus H-reflex (Meinck and Piesiur-Strehlow 1981, Delwaide and Crenna 1984, Kagamihara, Hayashi et al. 2003). As discussed in chapter three, the indirect methods used preclude direct identification of neuronal circuitry mediating the observed effects. Historically, similar studies have focused on reflex latency to determine the most likely pathway(s) utilized, however distance measurements used to determine conduction velocities must be approximated, and many assumptions must be made regarding central conduction velocities and the potential number of synapses involved. That being said, relative latencies in response to stimulation of different pathways appear to be consistent in non-injured subjects. It seems likely, given the extended period of facilitation and the presence of multiple 'peaks' of facilitation in response to ulnar nerve stimulation at latencies between $60 \mathrm{~ms}$ and $190 \mathrm{~ms}$, that the conditioning stimulation engaged multiple reflex pathways, with early and possibly middle latency $(<100 \mathrm{~ms})$ facilitation likely involving propriospinal pathways, and longer latency facilitation involving brain stem and cortical nuclei (Meinck and Piesiur-Strehlow 1981, Zehr, Collins et al. 2001). This interpretation is further supported by the preliminary results of our studies of the reticulospinal and vestibulospinal pathways, both of which resulted in conditioning of leg MMRs peaking at latencies of $110-140 \mathrm{~ms}$. Similarly, others investigating the reflex effects of these pathways on the soleus $\mathrm{H}$-reflex via galvanic vestibular stimulation (Kennedy and Inglis 2001) or acoustic startle 
(Rossignol and Jones 1976) found the strongest facilitation at 100ms and 110130ms, respectively.

Noteworthy differences with regard to laterality were not observed, however this may be due to the experimental conditions. As the participants were relaxed in supine, the need for functional coordination of the upper and lower limbs was not present. Examining MMR modulation in response to stimulation of upper limb peripheral nerves through different phases of the gait cycle may shed light on the functional relevance of the descending interlimb projections observed.

Although the descending tracts including corticospinal, reticulospinal, vestibulospinal, and descending propriospinal are known to make direct synaptic contact with motor neurons, they also project to interneurons in the intermediate grey and elsewhere (Brockett, Seenan et al. 2013). Interneurons residing in the intermediate grey are both excitatory and inhibitory (Liu, Bannatyne et al. 2010), however they receive much greater input from group I and group II afferents which comprises excitatory inputs, but can effect both excitation and facilitation of motor neurons. Many of the afferent projections onto the the interneuronal population are under strong inhibitory control by supraspinal projections, the relative level of which is dynamic, being modulated based on the task (Liu, Bannatyne et al. 2010). As such, the decrease in overall descending influence associated with the relaxed, supine position due to the lack of functional balance or postural requirements, may contribute to the preponderance of excitatory effects observed in this and similar studies. Similarly, damage to the descending 
tracts results in disinhibition of group I and II afferents, which can contribute to hypertonia and spasticity.

Still, it could be considered surprising that the MMR modulation observed was limited to excitation without an inhibitory component- although the longest condition-test interval tested was $300 \mathrm{~ms}$, and inhibitory effects beyond this latency cannot be ruled out.

Phasic modulation of the soleus $\mathrm{H}$-reflex has been investigating during different upper and lower lomb cycling paradigms, shown to involve interlimb reflexes (Frigon, Collins et al. 2004, de Ruiter, Hundza et al. 2010). However, these studies suggested that local cutaneous afferent input as well as central pattern generator acitivty might be responsible for the suppressive or inhibitory effects on specific motor pools at functionally relevant phases of gait as opposed to interlimb reflex effects. It is also notable that the similar interlimb reflex modulation was not seen in standing (Suzuki, Nakajima et al. 2016), as has been similarly reported by others (Dietz, Fouad et al. 2001). As was mentioned above with regard to the lack of left-right differences in MMR modulation, the simplest explanation might be that the supine test position was not ideal for the examination of functionally relevant interlimb interactions, and the general excitatory effects observed could have been a consequence of the nonphysiological and unfamiliar, abrupt sensory input induced by electrical stimulation of the ulnar nerve.

To the author's knowledge, 4 groups have studied descending interlimb reflexes extensively in neurologically intact humans- all began in a seated or 
supine relaxed position (Meinck and Piesiur-Strehlow 1981, Delwaide and Crenna 1984, Zehr, Collins et al. 2001, Kagamihara, Hayashi et al. 2003). None observed inhibitory effects on the soleus H-reflex in response to upper limb mixed or cutaneous nerve stimulation, which is generally analogous to the current experimental methods. However, 3 groups did not address the lack of inhibitory effects at all. Two also examined reflex responses in averaged EMG, and both found an early phase of inhibition (Meinck and Piesiur-Strehlow 1981, Kagamihara, Hayashi et al. 2003). Subjects were asked to maintain a constant low level of volitional activation during these experiments, as is typical in such studies, and the one author who addressed the difference in results between the two methods did not speculate beyond the conclusion that additional descending drive was required to induce inhibition (Meinck and Piesiur-Strehlow 1981). Given these results, it is also plausible that inhibition is not seen in evoked reflex modulation studies due to the fact that the motor neurons in such experiments are not under the influence of tonic excitatory influence, thus may be obscured by a 'floor effect'.

\section{Descending interlimb modulation of MMRs after SCI}

Eighteen participants with chronic spinal cord injury were assessed both for the presence of volitional muscle activation via FNPA, and for the presence of descending interlimb facilitation, identifying descending inter-enlargement spinal transmission. Results of interlimb MMR conditioning studies in motor complete SCI participants revealed descending facilitation of MMRs in 7/11 (64\%) individuals, providing evidence of residual descending systems capable of 
modulating lumbosacral motor neuron excitability which cannot be appreciated on clinical evaluation. However, when taking in account the level of injury, was observed that, in motor complete participants, descending interlimb modulation of leg MMRs almost exclusively occurred in participants with a lesion rostral to the C8 segment supplying the ulnar nerve, meaning descending conduction did not cross the injury zone. In the current study, $6 / 7 \mathrm{SCl}$ participants with a lesion above $\mathrm{C} 8$ demonstrated some MMR facilitation in response to ulnar nerve stimulation. In contrast, in only a single muscle in 1/4 participants injured caudal to C8 was MMR facilitation observed. When comparing the latencies at which MMR facilitation was first detected in motor complete participants, facilitation at early latencies represented $40 \%$ of all occurrences of MMR facilitation, with long latencies representing $37 \%$. In contrast, facilitation at early latencies $(60-90 \mathrm{~ms})$ represented $18 \%$ of the total number of observations in motor incomplete participants, while long latencies (140ms-300ms) represented $59 \%$. The greater percentage of early latency facilitation and decrease in long latency facilitation in motor complete participants injured above C8 may represent the expected sparing of inter-enlargement projections located caudal to the level of lesion, while the substantial loss of supraspinal input in these more severely injured participants is represented both by the decrease in the total number of reflex observations and the relative decrease in MMR facilitation at longer latencies.

Results of interlimb MMR conditioning studies in motor incomplete SCI participants revealed descending facilitation of MMRs in all individuals. It was remarkable that the location of the injury relative to the conditioning stimuli 
seemed to have little effect on the latencies at which MMR facilitation was first observed, although they frequently demonstrated the greatest magnitude of facilitation at longer latencies than motor complete participants. The variability in the results among subjects might be expected based on differences in level, mechanism, and severity of the injury. Additionally some of this variability may be related to the experimental methods, as some SCI participants had remarkable baseline variability in MMR amplitude at a given stimulus intensity, which may have masked the conditioning stimulation effects in those muscles. In particular this was the case for two of the motor incomplete subjects in which statistically significant MMR facilitation was only observed in one muscle each.

Ultimately, the goal was to identify spared descending spinal transmission capable of modulating lumbosacral motor excitability, which the current results establish. Although this is a small sample size, these results support the interpretation that MMR facilitation at early latencies represents a propriospinallymediated descending interlimb reflex which may be spared by injuries proximal to the lowest levels of the cervical enlargement, but is disrupted by severe $\mathrm{SCl}$ in the thoracic region. For individual subjects, comparison with results of other descending pathways studies including vestibulospinal, reticulospinal, and corticospinal pathways will allow more definitive conclusions to be drawn regarding the precise nature of the descending influence identified.

Voluntary muscle activation and interlimb MMR facilitation after spinal cord injury Long descending propriospinal neurons within the cervical enlargement have been shown to receive excitatory input from both reticulospinal and 
corticospinal tract fibers in mice, rats, cats, and primates (Alstermark, Lundberg et al. 1987, Rosenzweig, Courtine et al. 2010, Ni, Nawabi et al. 2014, Mitchell, McCallum et al. 2016), possibly providing an alternative route for supraspinal input which would allow integration of relevant afferent information 'en route' to the lumbosacral motor networks. As previously mentioned, animal models of SCl have also indicated a role for propriospinal networks in recovering supraspinalspinal connectivity after SCI (Bareyre, Kerschensteiner et al. 2004, Courtine, Song et al. 2008, Filli, Engmann et al. 2014). Based on these findings, the relationship between interlimb MMR facilitation and volitional leg muscle activation was also examined.

The FNPA allowed objective identification of volitional muscle activation, which was expressed as the ratio of active to baseline EMG amplitude. Seven of eighteen participants demonstrated volitional muscle activation of at least one leg muscle, indicating some residual neural connectivity to the lumbosacral motor circuitry caudal to the level of lesion. In comparing MMR conditioning studies and FNPA results, $73 \%$ of muscles in which volitional activation was observed also demonstrated MMR facilitation in response to ulnar nerve stimulation. Similarly $83 \%$ of muscles in which volitional activation was not observed also showed no statistically significant change in MMR amplitude in response to ulnar nerve stimulation. As discussed in the previous chapter, although the group results showed a significant relationship $(p<.0001)$, the results were variable among those participants with volitional activation. In particular, 4 participants, who were all full time community ambulators with similar levels of functional independence, 
were able to volitionally activate all lower extremity muscles. However MMR facilitation of all muscles was observed in only two of these participants, while the remaining two only demonstrated MMR conditioning in just one muscle each. Such a discrepancy between individuals with similar levels of function can most probably be attributed to differences in the location and mechanism of injury, and the individualized reorganization of the spared circuitry.

\section{Limitations}

Interlimb modulation studies in SCI participants were difficult to analyze and interpret. The primary methodological limitations included the inability to directly identify the route of transmission, and increased variability in responses observed in SCl participants, presumably related to pathological changes in sublesional spinal circuits. Analysis of the variability in the control responses revealed increased variability in these subjects, which in some instances revealed longer lasting effects on motor neuron excitability from a single stimulation than were observed in the non-injured participants. This may have played a role in the incomplete participants in whom no conditioning was observed. In addition, while we attempted to target the ideal test MMR amplitude in each muscle for conditioning studies, this was not always possible in all subjects due to pathological differences in MMR recruitment thresholds among muscles as well as time constraints. For these reasons, it is possible that methodological limitations can account for the lack of modulation in some muscles demonstrating volitional activation. 
Additionally, while we insured that all participants had not participated in any therapeutic activities and were not taking any anti-spasticity medications the day of the assessment, we did not control for the time of day or diet, both which might have influenced the overall physiological state of nervous system.

\section{Future Directions}

The current study represents a first step in the direction of pathway specific spinal cord injury assessment. Several methodological modifications may be recommended for future studies of the putative inter-enlargement propriospinal pathways in humans. Lingering questions regarding the involvement of supraspinal relays in interlimb reflexes may be more directly assessed by electrical stimulation of both enlargements in the same conditiontest paradigm as used in the current study. This simplifies the calculation of central conduction by removing peripheral conduction from the equation, and movement of either electrode rostro-caudally along the spinal cord could provide the most definitive evidence to date of the existence (or lack thereof) of both long ascending and long descending propriospinal neurons in humans. The proposed methodology might further be improved by the use of pulse trains and/or continuous stimulation as both test and conditioning stimuli.

Simultaneous stimulation of both enlargments in therapeutic stimulation paradigms is also intriguing. It might be hypothesized that sub-motor threshold cervical spinal stimulation, in a similar manner to what is observed with lumbosacral stimulation, could raise the excitability level of local cervical networks through the increased afferent input, and allowing more efficient and 
effective relay of supraspinal inputs via long descending propriospinal neurons residing in these segments.

Spinal stimulation frequency in the current study was $1 / 6 \mathrm{sec}$, or $.167 \mathrm{~Hz}$. In contrast, epidural spinal stimulation frequencies reported to promote postural muscle activation in standing or volitional movement in paralyzed muscles are typically 25-40Hz (Angeli, Edgerton et al. 2014, Rejc, Angeli et al. 2015). As such, future studies should also assess conditioning stimuli while lumbosacral spinal stimulation delivered at these more functionally relevant frequencies. Interestingly, despite the very low stimulation frequency used in the current study, a large number of participants reported a subjective decrease in spasticity and/or neuropathic pain which persisted for several hours following the experiment. At present only preliminary reports exist on the effects of transcutaneous spinal stimulation on spasticity and neuropathic pain, and this line of investigation seems intriguing, especially given the scarcity of effective non-pharmacological treatment strategies for either condition.

While a single index of motor function might summarize the extent of residual volitional motor function in individuals with $\mathrm{SCl}$, it fails to effectively characterize the capacity of the spinal cord to effectively integrate sensory information and to generate appropriate motor behaviors (Sherwood, Dimitrijevic et al. 1992). One of the primary reasons for this is the nature of the testing conditions under which the assessment(s) is/are carried out (Maegele, Muller et al. 2002, Forrest, Sisto et al. 2008). As such, studies of descending pathways in functionally relevant conditions may yield different results. Recent studies of 
propriospinal function in humans have frequently utilized similar conditioning paradigms during walking or cycling of the upper and/or lower extremities. Such conditions require arm and leg coordination, so the presence or absence of phase modulation as well as the functional latencies would add to the interpretation of the nature of the descending facilitation. Use of the current and proposed conditioning paradigms in these more functionally relevant circumstances can further elucidate the extent to which a functional long propriospinal system and/or other descending systems are involved in producing task-specific muscle activation seen during standing and stepping, as well as volitional movement. Conditioning stimulation applied during the performance of these tasks in the presence of continuous spinal stimulation would provide the most direct information regarding their roles, in muscle activity observed. Further, once residual and or de novo pathways are identified, comparison with clinical outcomes could provide additional insights into the role or the respective descending tracts in the clinical presentation of individual subjects. For example, clinical presentation of hypertonicity or 'spasticity' as it is usually referred to the clinical setting, can vary greatly among individuals in terms of local vs. global effects, severity, and variability throughout the sleep-wake cycle and in different functional positions. While it has been hypothesized that increased hypertonicity during standing and gait is related to residual vestibulospinal and reticulospinal inputs which control posture and gait to a large extent, these and similar hypotheses could be more directly examined using the described techniques. 
Several studies have shown that paired-associative stimulation can strengthen existing neural synapses via Hebbian mechanisms (Possover 2014, Dixon, Ibrahim et al. 2016). In the current study, a facilitative effect was seen at the spinal motor neurons following stimulation of both the ulnar nerve and the lumbosacral spinal cord at the appropriate latency. Future studies should seek to determine whether repetitive dual-site stimulation can strengthen these connections over time. Stimulation of both upper and lower limbs during ambulation might serve to strengthen descending intralimb inputs, thus improving the overall locomotor neuromuscular and kinematic patterns. This technique has the advantage of incorporated the paired associative stimulation techniques, providing excitation both above and below the the level of lesion except in high cervical lesions, into a activities-based intervention which also engages other supraspinal-spinal tracts in the maintenance of balance and posture during the task.

In particular, combining multi-muscle neuro-muscular electrical stimulation (NMES) with spinal stimulation could promote muscle activation sufficient for standing/stepping at much lower stimulation intensities than either NMES or spinal stimulation applied alone. This would of particular importance to training paradigms incorporating transcutaneous spinal stimulation in patients with intact sensation and/or risk of autonomic dysreflexia.

As discussed, altering the functional state of the spinal cord via spinal stimulation at sub-motor threshold intensities allows the lumbosacral circuitry to produce functional muscle activation patterns in response to relevant 
proprioceptive input; however even when using multiple sites of spinal stimulation and/or multiple spinal stimulation configurations concurrently, the overall muscle output that is produced, in particular the relative balance between flexors and extensors, can vary by participant, and by limb or joint within a participant. To address these asymetries, NMES could be used to augment the activation in specific muscles where needed, introducing additional level of customization to the stimulation for use in standing, stepping, or volitional movement training. Not only could NMES be applied at different intensities to individual muscles, but the on/off timing of this stimulation could be varied by muscle as well, which would be advantageous for gait training as well as volitional movement training.

Also of note, while the presence of descending volitional control and/or modulation of motor output in the presence of spinal electrical stimulation has received much well deserved interest, the effects of such stimulation on the ongoing ascending output from the spinal cord should not be overlooked. Spinal stimulation provided transcutaneously or epidurally is applied over the dorsal aspect of the spinal cord, and the effects on local networks' excitability states are mediated at least in part by stimulation of dorsal root afferents (Rattay, Minassian et al. 2000, Ladenbauer, Minassian et al. 2010, Danner, Hofstoetter et al. 2011). Whether the sensory input to supraspinal centers is 1) altered in the presence of spinal electrical stimulation and 2) required for the re-emergence of volitional movement are important questions which need to be investigated. 


\section{REFERENCES}

(2008). "Early acute management in adults with spinal cord injury: a clinical practice guideline for health-care professionals." J Spinal Cord Med 31(4): 403-479.

Alstermark, B., T. Isa, L. G. Pettersson and S. Sasaki (2007). "The C3-C4 propriospinal system in the cat and monkey: a spinal pre-motoneuronal centre for voluntary motor control." Acta Physiol (Oxf) 189(2): 123-140.

Alstermark, B., A. Lundberg, M. Pinter and S. Sasaki (1987). "Long C3-C5 propriospinal neurones in the cat." Brain Res 404(1-2): 382-388.

Alstermark, B., J. Ogawa and T. Isa (2004). "Lack of monosynaptic corticomotoneuronal EPSPs in rats: disynaptic EPSPs mediated via reticulospinal neurons and polysynaptic EPSPs via segmental interneurons." J Neurophysiol 91(4): 1832-1839.

Alstermark, B., L. G. Pettersson, Y. Nishimura, K. Yoshino-Saito, F. Tsuboi, M. Takahashi and T. Isa (2011). "Motor command for precision grip in the macaque monkey can be mediated by spinal interneurons." $\underline{\mathrm{J}}$ Neurophysiol 106(1): 122-126.

Angeli, C. A., V. R. Edgerton, Y. P. Gerasimenko and S. J. Harkema (2014). "Altering spinal cord excitability enables voluntary movements after chronic complete paralysis in humans." Brain 137(Pt 5): 1394-1409.

Asaka, T., Y. Wang, J. Fukushima and M. L. Latash (2008). "Learning effects on muscle modes and multi-mode postural synergies." Exp Brain Res 184(3): 323-338. 
Bachmann, L. C., N. T. Lindau, P. Felder and M. E. Schwab (2014). "Sprouting of brainstem-spinal tracts in response to unilateral motor cortex stroke in mice." J Neurosci 34(9): 3378-3389.

Ballion, B., D. Morin and D. Viala (2001). "Forelimb locomotor generators and quadrupedal locomotion in the neonatal rat." Eur J Neurosci 14(10): 17271738.

Bareyre, F. M., M. Kerschensteiner, O. Raineteau, T. C. Mettenleiter, O. Weinmann and M. E. Schwab (2004). "The injured spinal cord spontaneously forms a new intraspinal circuit in adult rats." Nat Neurosci 7(3): 269-277.

Barthelemy, D., M. Willerslev-Olsen, H. Lundell, F. Biering-Sorensen and J. B. Nielsen (2015). "Assessment of transmission in specific descending pathways in relation to gait and balance following spinal cord injury." Prog Brain Res 218: 79-101.

Behrman, A. L., E. Ardolino, L. R. Vanhiel, M. Kern, D. Atkinson, D. J. Lorenz and S. J. Harkema (2012). "Assessment of functional improvement without compensation reduces variability of outcome measures after human spinal cord injury." Arch Phys Med Rehabil 93(9): 1518-1529.

Benjamini, Y. and Y. Hochberg (1995). "Controlling the False Discovery Rate: A Practical and Powerful Approach to Multiple Testing." Journal of the Royal Statistical Society. Series B (Methodological) 57(1): 289-300.

Brockett, E. G., P. G. Seenan, B. A. Bannatyne and D. J. Maxwell (2013). "Ascending and descending propriospinal pathways between lumbar and 
cervical segments in the rat: evidence for a substantial ascending excitatory pathway." Neuroscience 240: 83-97.

Bucy, P. C., J. E. Keplinger and E. B. Siqueira (1964). "DESTRUCTION OF THE

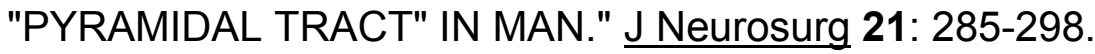

Bucy, P. C., R. Ladpli and A. Ehrlich (1966). "Destruction of the pyramidal tract in the monkey. The effects of bilateral section of the cerebral peduncles." $\underline{\mathrm{J}}$ Neurosurg 25(1): 1-23.

Buehner, J. J., G. F. Forrest, M. Schmidt-Read, S. White, K. Tansey and D. M. Basso (2012). "Relationship between ASIA examination and functional outcomes in the NeuroRecovery Network Locomotor Training Program." Arch Phys Med Rehabil 93(9): 1530-1540.

Bunge, R., W. R. Puckett, J. L. Becerra, A. Marcillo and R. M. Quencer (1993). "Observations on the pathology of human spinal cord injury." Adv Neurol 59: 75-89.

Bunge, R. P., W. R. Puckett, J. L. Becerra, A. Marcillo and R. M. Quencer (1993). "Observations on the pathology of human spinal cord injury. A review and classification of 22 new cases with details from a case of chronic cord compression with extensive focal demyelination." Adv Neurol 59: 75-89.

Butler, J. E., S. Godfrey and C. K. Thomas (2016). "Interlimb Reflexes Induced by Electrical Stimulation of Cutaneous Nerves after Spinal Cord Injury." PLoS One 11(4): e0153063.

Calancie, B. (1991). "Interlimb reflexes following cervical spinal cord injury in man." Exp Brain Res 85(2): 458-469. 
Calancie, B., N. Alexeeva, J. G. Broton and M. R. Molano (2005). "Interlimb reflex activity after spinal cord injury in man: strengthening response patterns are consistent with ongoing synaptic plasticity." Clin Neurophysiol 116(1): 75-86.

Calancie, B., N. Alexeeva, J. G. Broton, S. Suys, A. Hall and K. J. Klose (1999). "Distribution and latency of muscle responses to transcranial magnetic stimulation of motor cortex after spinal cord injury in humans." $\underline{\mathrm{J}}$ Neurotrauma 16(1): 49-67.

Calancie, B., S. Lutton and J. G. Broton (1996). "Central nervous system plasticity after spinal cord injury in man: interlimb reflexes and the influence of cutaneous stimulation." Electroencephalogr Clin Neurophysiol 101(4): 304-315.

Cioni, B., M. R. Dimitrijevic, W. B. McKay and A. M. Sherwood (1986). "Voluntary supraspinal suppression of spinal reflex activity in paralyzed muscles of spinal cord injury patients." Exp Neurol 93(3): 574-583.

Conta Steencken, A. C. and D. J. Stelzner (2010). "Loss of propriospinal neurons after spinal contusion injury as assessed by retrograde labeling." Neuroscience 170(3): 971-980.

Courtine, G., S. J. Harkema, C. J. Dy, Y. P. Gerasimenko and P. Dyhre-Poulsen (2007). "Modulation of multisegmental monosynaptic responses in a variety of leg muscles during walking and running in humans." $\underline{\mathrm{J} \text { Physiol }}$ 582(Pt 3): 1125-1139. 
Courtine, G., B. Song, R. R. Roy, H. Zhong, J. E. Herrmann, Y. Ao, J. Qi, V. R. Edgerton and M. V. Sofroniew (2008). "Recovery of supraspinal control of stepping via indirect propriospinal relay connections after spinal cord injury." Nat Med 14(1): 69-74.

Cowley, K. C., B. J. MacNeil, J. W. Chopek, S. Sutherland and B. J. Schmidt (2015). "Neurochemical excitation of thoracic propriospinal neurons improves hindlimb stepping in adult rats with spinal cord lesions." Exp Neurol 264: 174-187.

Cowley, K. C., E. Zaporozhets and B. J. Schmidt (2010). "Propriospinal transmission of the locomotor command signal in the neonatal rat." Ann $\mathrm{N}$ Y Acad Sci 1198: 42-53.

Crone, C., H. Hultborn, L. Mazieres, C. Morin, J. Nielsen and E. PierrotDeseilligny (1990). "Sensitivity of monosynaptic test reflexes to facilitation and inhibition as a function of the test reflex size: a study in man and the cat." Exp Brain Res 81(1): 35-45.

Curt, A., M. E. Keck and V. Dietz (1998). "Functional outcome following spinal cord injury: significance of motor-evoked potentials and ASIA scores." Arch Phys Med Rehabil 79(1): 81-86.

Danner, S. M., U. S. Hofstoetter, J. Ladenbauer, F. Rattay and K. Minassian (2011). "Can the human lumbar posterior columns be stimulated by transcutaneous spinal cord stimulation? A modeling study." Artif Organs 35(3): 257-262. 
De Gail, P., J. W. Lance and P. D. Neilson (1966). "Differential effects on tonic and phasic reflex mechanisms produced by vibration of muscles in man." J Neurol Neurosurg Psychiatry 29(1): 1-11.

de Ruiter, G. C., S. R. Hundza and E. P. Zehr (2010). "Phase-dependent modulation of soleus H-reflex amplitude induced by rhythmic arm cycling." Neurosci Lett 475(1): 7-11.

Delwaide, P. J. and P. Crenna (1984). "Cutaneous nerve stimulation and motoneuronal excitability. II: Evidence for non-segmental influences." $\underline{\mathrm{J}}$ Neurol Neurosurg Psychiatry 47(2): 190-196.

Delwaide, P. J. and B. Schepens (1995). "Auditory startle (audio-spinal) reaction in normal man: $\mathrm{EMG}$ responses and $\mathrm{H}$ reflex changes in antagonistic lower limb muscles." Electroencephalogr Clin Neurophysiol 97(6): 416423.

Dietz, V., K. Fouad and C. M. Bastiaanse (2001). "Neuronal coordination of arm and leg movements during human locomotion." Eur J Neurosci 14(11): 1906-1914.

Dietz, V. and J. Michel (2009). "Human bipeds use quadrupedal coordination during locomotion." Ann N Y Acad Sci 1164: 97-103.

Dimitrijevic, M., C. Y. Hsu and W. B. McKay (1992). "Neurophysiological assessment of spinal cord and head injury." J Neurotrauma 9: S293-300. Dimitrijevic, M. R., M. M. Dimitrijevic, J. Faganel and A. M. Sherwood (1984). "Suprasegmentally induced motor activity in paralyzed muscles of patients with established spinal cord injury." Ann Neurol 16(2): 2116-2221. 
Dimitrijevic, M. R., M. M. Dimitrijevic, J. Faganel and A. M. Sherwood (1984). "Suprasegmentally induced motor unit activity in paralyzed muscles of patients with established spinal cord injury." Ann Neurol 16(2): 216-221.

Dimitrijevic, M. R., C. Y. Hsu and W. B. McKay (1992). "Neurophysiological assessment of spinal cord and head injury." J Neurotrauma 9 Suppl 1: S293-300.

Dimitrijevic, M. R., W. A. Spencer, J. V. Trontelj and M. Dimitrijevic (1977). "Reflex effects of vibration in patients with spinal cord lesions." Neurology 27(11): 1078-1086.

Dixon, L., M. M. Ibrahim, D. Santora and M. Knikou (2016). "Paired Associative Transspinal and Transcortical Stimulation Produces Plasticity in Human Cortical and Spinal Neuronal Circuits." J Neurophysiol: jn.00259.02016.

Dragert, K. and E. P. Zehr (2009). "Rhythmic arm cycling modulates Hoffmann reflex excitability differentially in the ankle flexor and extensor muscles." Neurosci Lett 450(3): 235-238.

Du Beau, A., S. Shakya Shrestha, B. A. Bannatyne, S. M. Jalicy, S. Linnen and D. J. Maxwell (2012). "Neurotransmitter phenotypes of descending systems in the rat lumbar spinal cord." Neuroscience 227: 67-79.

Dy, C. J., Y. P. Gerasimenko, V. R. Edgerton, P. Dyhre-Poulsen, G. Courtine and S. J. Harkema (2010). "Phase-dependent modulation of percutaneously elicited multisegmental muscle responses after spinal cord injury." $\underline{\mathrm{J}}$ Neurophysiol 103(5): 2808-2820. 
Edgerton, V. R., J. L. Smith and D. R. Simpson (1975). "Muscle fibre type populations of human leg muscles." Histochem J 7(3): 259-266.

Ellaway, P. H., A. Kuppuswamy, A. V. Balasubramaniam, R. Maksimovic, A. Gall, M. D. Craggs, C. J. Mathias, M. Bacon, A. Prochazka, J. Kowalczewski, B. A. Conway, S. Galen, C. J. Catton, D. B. Allan, A. Curt, B. Wirth and H. J. van Hedel (2011). "Development of quantitative and sensitive assessments of physiological and functional outcome during recovery from spinal cord injury: a clinical initiative." Brain Res Bull 84(4-5): 343357.

Filli, L., A. K. Engmann, B. Zorner, O. Weinmann, T. Moraitis, M. Gullo, H. Kasper, R. Schneider and M. E. Schwab (2014). "Bridging the gap: a reticulo-propriospinal detour bypassing an incomplete spinal cord injury." $\underline{\mathrm{J}}$ Neurosci 34(40): 13399-13410.

Forrest, G. F., S. A. Sisto, H. Barbeau, S. C. Kirshblum, J. Wilen, Q. Bond, S. Bentson, P. Asselin, C. M. Cirnigliaro and S. Harkema (2008). "Neuromotor and musculoskeletal responses to locomotor training for an individual with chronic motor complete AIS-B spinal cord injury." $\underline{\mathrm{J} \text { Spinal }}$ Cord Med 31(5): 509-521.

Frigon, A., D. F. Collins and E. P. Zehr (2004). "Effect of rhythmic arm movement on reflexes in the legs: modulation of soleus $\mathrm{H}$-reflexes and somatosensory conditioning." J Neurophysiol 91(4): 1516-1523.

Gerasimenko, Y., P. Musienko, I. Bogacheva, T. Moshonkina, A. Savochin, I. Lavrov, R. R. Roy and V. R. Edgerton (2009). "Propriospinal bypass of the 


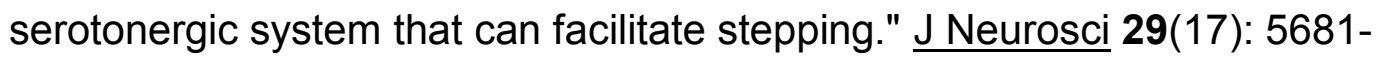
5689.

Gerasimenko, Y. P., D. C. Lu, M. Modaber, S. Zdunowski, P. Gad, D. G. Sayenko, E. Morikawa, P. Haakana, A. R. Ferguson, R. R. Roy and V. R. Edgerton (2015). "Noninvasive Reactivation of Motor Descending Control after Paralysis." $\underline{\mathrm{J} \text { Neurotrauma. }}$

Gillies, J. D., D. J. Burke and J. W. Lance (1971). "The supraspinal control of the tonic vibration reflex." Proc Aust Assoc Neurol 8: 143-146.

Gillies, J. D., D. J. Burke and J. W. Lance (1971). "Tonic vibration reflex in the cat." J Neurophysiol 34(2): 252-262.

Gorodnichev, R. M., E. A. Pivovarova, A. Pukhov, S. A. Moiseev, A. A. Savokhin, T. R. Moshonkina, N. A. Shcherbakova, V. A. Kilimnik, V. A. Selionov, I. B. Kozlovskaia, V. R. Edgerton and P. Gerasimenko lu (2012).

"[Transcutaneous electrical stimulation of the spinal cord: non-invasive tool for activation of locomotor circuitry in human]." Fiziol Cheloveka 38(2): 46-56.

Graves, D. E., R. G. Frankiewicz and W. H. Donovan (2006). "Construct validity and dimensional structure of the ASIA motor scale." $\underline{\mathrm{J} \text { Spinal Cord Med }}$ 29(1): 39-45.

Harkema, S., Y. Gerasimenko, J. Hodes, J. Burdick, C. Angeli, Y. Chen, C. Ferreira, A. Willhite, E. Rejc, R. G. Grossman and V. R. Edgerton (2011). "Effect of epidural stimulation of the lumbosacral spinal cord on voluntary 
movement, standing, and assisted stepping after motor complete paraplegia: a case study." The Lancet 377(9781): 1938-1947.

Harkema, S., Y. Gerasimenko, J. Hodes, J. Burdick, C. Angeli, Y. Chen, C.

Ferreira, A. Willhite, E. Rejc, R. G. Grossman and V. R. Edgerton (2011).

"Effect of epidural stimulation of the lumbosacral spinal cord on voluntary

movement, standing, and assisted stepping after motor complete

paraplegia: a case study." Lancet 377(9781): 1938-1947.

Hofstoetter, U. S., K. Minassian, C. Hofer, W. Mayr, F. Rattay and M. R.

Dimitrijevic (2008). "Modification of reflex responses to lumbar posterior root stimulation by motor tasks in healthy subjects." Artif Organs 32(8): 644-648.

Isa, T., Y. Ohki, B. Alstermark, L. G. Pettersson and S. Sasaki (2007). "Direct and indirect cortico-motoneuronal pathways and control of hand/arm movements." Physiology (Bethesda) 22: 145-152.

Jaberzadeh, S., M. Zoghi, P. Morgan and M. Storr (2013). "Corticospinal

Facilitation of Erector Spinae and Rectus Abdominis Muscles During Graded Voluntary Contractions is Task Specific: A Pilot Study on Healthy Individuals." Basic Clin Neurosci 4(3): 209-216.

Juvin, L., J. Simmers and D. Morin (2005). "Propriospinal circuitry underlying interlimb coordination in mammalian quadrupedal locomotion." $\underline{\mathrm{J} \text { Neurosci }}$ 25(25): 6025-6035. 
Kagamihara, Y., A. Hayashi, Y. Masakado and Y. Kouno (2003). "Long-loop reflex from arm afferents to remote muscles in normal man." Exp Brain Res 151(1): 136-144.

Kakulas, B. A. (1999). "The applied neuropathology of human spinal cord injury." Spinal Cord 37(2): 79-88.

Kakulas, B. A. (1999). "A review of the neuropathology of human spinal cord injury with emphasis on special features." J Spinal Cord Med 22(2): 119124.

Kakulas, B. A. and C. Kaelan (2015). "The neuropathological foundations for the restorative neurology of spinal cord injury." Clin Neurol Neurosurg 129 Suppl 1: S1-7.

Kendall, F. P., E. K. McCreary and H. O. Kendall (1983). Muscles, testing and function. Baltimore, Williams \& Wilkins.

Kennedy, P. M. and J. T. Inglis (2001). "Modulation of the soleus H-reflex in prone human subjects using galvanic vestibular stimulation." $\underline{\text { Clin }}$ Neurophysiol 112(11): 2159-2163.

Kirshblum, S. and W. Waring, 3rd (2014). "Updates for the International Standards for Neurological Classification of Spinal Cord Injury." Phys Med Rehabil Clin N Am 25(3): 505-517, vii.

Kirshblum, S. C. and K. C. O'Connor (1998). "Predicting neurologic recovery in traumatic cervical spinal cord injury." Arch Phys Med Rehabil 79(11): 1456-1466. 
Kirshblum, S. C., W. Waring, F. Biering-Sorensen, S. P. Burns, M. Johansen, M. Schmidt-Read, W. Donovan, D. Graves, A. Jha, L. Jones, M. J. Mulcahey and A. Krassioukov (2011). "Reference for the 2011 revision of the International Standards for Neurological Classification of Spinal Cord Injury." J Spinal Cord Med 34(6): 547-554.

Ladenbauer, J., K. Minassian, U. S. Hofstoetter, M. R. Dimitrijevic and F. Rattay (2010). "Stimulation of the human lumbar spinal cord with implanted and surface electrodes: a computer simulation study." IEEE Trans Neural Syst Rehabil Eng 18(6): 637-645.

Lavrov, I., C. J. Dy, A. J. Fong, Y. Gerasimenko, G. Courtine, H. Zhong, R. R. Roy and V. R. Edgerton (2008). "Epidural stimulation induced modulation

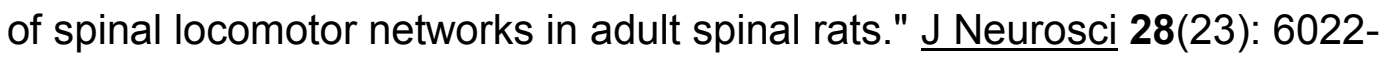
6029 .

Lee, D. C., H. K. Lim, W. B. McKay, M. M. Priebe, S. A. Holmes and A. M. Sherwood (2004). "Toward an objective interpretation of surface EMG

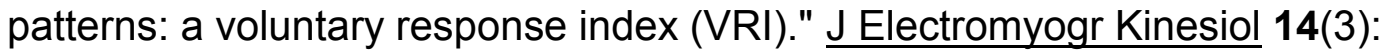
379-388.

Lemon, R. N. (2008). "Descending pathways in motor control." Annu Rev Neurosci 31: 195-218.

Li, K., D. Atkinson, M. Boakye, C. Z. Tolfo, S. Aslan, M. Green, B. McKay, A. Ovechkin and S. J. Harkema (2012). "Quantitative and sensitive assessment of neurophysiological status after human spinal cord injury." $\underline{\mathrm{J}}$ Neurosurg Spine 17(1 Suppl): 77-86. 
Lim, H. K., D. C. Lee, W. B. McKay, M. M. Priebe, S. A. Holmes and A. M. Sherwood (2005). "Neurophysiological assessment of lower-limb voluntary control in incomplete spinal cord injury." Spinal Cord 43(5): 283-290.

Liu, T. T., B. A. Bannatyne, E. Jankowska and D. J. Maxwell (2010). "Properties of axon terminals contacting intermediate zone excitatory and inhibitory premotor interneurons with monosynaptic input from group I and II muscle afferents." J Physiol 588(Pt 21): 4217-4233.

Lloyd, D. and A. McIntyre (1948). "Analysis of forelimb-hindlimb reflex activity in acutely decapitate cats." $\underline{\mathrm{J} \text { Neurophysiol }}$ 11(5): 455-470.

Maegele, M., S. Muller, A. Wernig, V. R. Edgerton and S. J. Harkema (2002). "Recruitment of spinal motor pools during voluntary movements versus stepping after human spinal cord injury." J Neurotrauma 19(10): 12171229.

Magladery, J. W. and D. D. Mc, Jr. (1950). "Electrophysiological studies of nerve and reflex activity in normal man. I. Identification of certain reflexes in the electromyogram and the conduction velocity of peripheral nerve fibers." Bull Johns Hopkins Hosp 86(5): 265-290.

Magladery, J. W., W. E. Porter, A. M. Park and R. D. Teasdall (1951). "Electrophysiological studies of nerve and reflex activity in normal man. IV. The two-neurone reflex and identification of certain action potentials from spinal roots and cord." Bull Johns Hopkins Hosp 88(6): 499-519. 
Marchand-Pauvert, V., M. Simonetta-Moreau and E. Pierrot-Deseilligny (1999). "Cortical control of spinal pathways mediating group II excitation to human thigh motoneurones." $\underline{\text { J Physiol }} 517$ ( Pt 1): 301-313.

Marino, R. J., T. Barros, F. Biering-Sorensen, S. P. Burns, W. H. Donovan, D. E. Graves, M. Haak, M. A. Hudson and M. M. Priebe (2003). "International Standards for Neurological Classification of Spinal Cord Injury." j spinal cord med 26: S50-S56.

Marino, R. J. and D. E. Graves (2004). "Metric properties of the ASIA motor score: Subscales improve correlation with functional activities." Archives of Physical Medicine and Rehabilitation 85(11): 1804-1810.

Marino, R. J., L. Jones, S. Kirshblum, J. Tai and A. Dasgupta (2008). "repeatability and reliability of the motor and sensory examination of the international standards for neurological classification of spinal cord injury." J spinal cord med 11(2): 166-170.

Matsuyama, K., F. Mori, K. Nakajima, T. Drew, M. Aoki and S. Mori (2004).

"Locomotor role of the corticoreticular-reticulospinal-spinal interneuronal system." Prog Brain Res 143: 239-249.

McKay, W. B., D. C. Lee, H. K. Lim, S. A. Holmes and A. M. Sherwood (2005). "Neurophysiological examination of the corticospinal system and voluntary motor control in motor-incomplete human spinal cord injury." Exp Brain Res 163(3): 379-387. 
McKay, W. B., H. K. Lim, M. M. Priebe, D. S. Stokic and A. M. Sherwood (2004). "Clinical neurophysiological assessment of residual motor control in postspinal cord injury paralysis." Neurorehabil Neural Repair 18(3): 144-153.

McKay, W. B., A. V. Ovechkin, T. W. Vitaz, D. G. Terson de Paleville and S. J. Harkema (2011). "Long-lasting involuntary motor activity after spinal cord injury." Spinal Cord 49(1): 87-93.

McKay, W. B., A. V. Ovechkin, T. W. Vitaz, D. G. Terson de Paleville and S. J. Harkema (2011). "Neurophysiological characterization of motor recovery in acute spinal cord injury." Spinal Cord 49(3): 421-429.

Meinck, H.-M. and B. Piesiur-Strehlow (1981). "Reflexes evoked in leg muscles from arm afferents: a propriospinal pathway in man?" Exp Brain Res 43: 78-86.

Meinck, H. M. and B. Piesiur-Strehlow (1981). "Reflexes evoked in leg muscles from arm afferents: a propriospinal pathway in man?" Exp Brain Res 43(1): 78-86.

Meunier, S., E. Pierrot-Deseilligny and M. Simonetta-Moreau (1994). "Pattern of heteronymous recurrent inhibition in the human lower limb." Exp Brain Res 102(1): 149-159.

Middleton, J. W., G. Truman and T. J. Geraghty (1998). "Neurological level effect on the discharge functional status of spinal cord injured persons after rehabilitation." Arch Phys Med Rehabil 79(11): 1428-1432. 
Minassian, K., I. Persy, F. Rattay, M. R. Dimitrijevic, C. Hofer and H. Kern (2007). "Posterior root-muscle reflexes elicited by transcutaneous stimulation of the human lumbosacral cord." Muscle Nerve 35(3): 327-336.

Mitchell, E. J., S. McCallum, D. Dewar and D. J. Maxwell (2016). "Corticospinal and Reticulospinal Contacts on Cervical Commissural and Long Descending Propriospinal Neurons in the Adult Rat Spinal Cord; Evidence for Powerful Reticulospinal Connections." PLoS One 11(3): e0152094.

Nathan, P. W. (1994). "Effects on movement of surgical incisions into the human spinal cord." Brain 117 ( Pt 2): 337-346.

Nathan, P. W., M. Smith and P. Deacon (1996). "Vestibulospinal, reticulospinal and descending propriospinal nerve fibres in man." Brain 119 (Pt 6): 1809-1833.

Ni, Y., H. Nawabi, X. Liu, L. Yang, K. Miyamichi, A. Tedeschi, B. Xu, N. R. Wall, E. M. Callaway and Z. He (2014). "Characterization of long descending

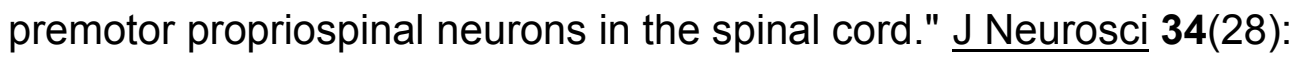
9404-9417.

Pierrot-Deseilligny, E. (1989). "Peripheral and descending control of neurones mediating non-monosynaptic la excitation to motoneurones: a presumed propriospinal system in man." Prog Brain Res 80: 305-314; discussion 295-307.

Pierrot-Deseilligny, E. (2002). "Propriospinal transmission of part of the corticospinal excitation in humans." Muscle Nerve 26(2): 155-172. 
Pierrot-Deseilligny, E. (2005). "The Circuitry of the Human Spinal Cord: its role in motor control and movement disorders." Cambridge University Press, New York: 1-642.

Piesiur-Strehlow, B. and H. M. Meinck (1980). "Response patterns of human lumbo-sacral motoneurone pools to distant somatosensory stimuli." Electroencephalogr Clin Neurophysiol 48(6): 673-682.

Pinheiro, J. C. B., D.M. (2000). Effects Models in S and S-Plus. Verlag, NY, Springer.

Possover, M. (2014). "Recovery of sensory and supraspinal control of leg movement in people with chronic paraplegia: a case series." Arch Phys Med Rehabil 95(4): 610-614.

Rattay, F., K. Minassian and M. R. Dimitrijevic (2000). "Epidural electrical stimulation of posterior structures of the human lumbosacral cord: 2 . quantitative analysis by computer modeling." Spinal Cord 38(8): 473-489.

Reed, W. R., A. Shum-Siu, S. M. Onifer and D. S. Magnuson (2006). "Interenlargement pathways in the ventrolateral funiculus of the adult rat spinal cord." Neuroscience 142(4): 1195-1207.

Rejc, E., C. Angeli and S. Harkema (2015). "Effects of Lumbosacral Spinal Cord Epidural Stimulation for Standing after Chronic Complete Paralysis in Humans." PLoS One 10(7): e0133998.

Rosenzweig, E. S., G. Courtine, D. L. Jindrich, J. H. Brock, A. R. Ferguson, S. C. Strand, Y. S. Nout, R. R. Roy, D. M. Miller, M. S. Beattie, L. A. Havton, J. C. Bresnahan, V. R. Edgerton and M. H. Tuszynski (2010). "Extensive 
spontaneous plasticity of corticospinal projections after primate spinal cord injury." Nat Neurosci 13(12): 1505-1510.

Rossignol, S. and G. M. Jones (1976). "Audio-spinal influence in man studied by the H-reflex and its possible role on rhythmic movements synchronized to sound." Electroencephalogr Clin Neurophysiol 41(1): 83-92.

Rossini, P. M., D. Burke, R. Chen, L. G. Cohen, Z. Daskalakis, R. Di lorio, V. Di Lazzaro, F. Ferreri, P. B. Fitzgerald, M. S. George, M. Hallett, J. P. Lefaucheur, B. Langguth, H. Matsumoto, C. Miniussi, M. A. Nitsche, A. Pascual-Leone, W. Paulus, S. Rossi, J. C. Rothwell, H. R. Siebner, Y. Ugawa, V. Walsh and U. Ziemann (2015). "Non-invasive electrical and magnetic stimulation of the brain, spinal cord, roots and peripheral nerves: Basic principles and procedures for routine clinical and research application. An updated report from an I.F.C.N. Committee." $\underline{\text { Clin }}$ Neurophysiol 126(6): 1071-1107.

Roy, F. D., D. Bosgra and R. B. Stein (2014). "Interaction of transcutaneous spinal stimulation and transcranial magnetic stimulation in human leg muscles." Exp Brain Res 232(6): 1717-1728.

Roy, F. D., G. Gibson and R. B. Stein (2012). "Effect of percutaneous stimulation at different spinal levels on the activation of sensory and motor roots." Exp Brain Res 223(2): 281-289.

Sable, A. W. (1998). "Median and ulnar nerves in the hand." Phys Med Rehabil Clin N Am 9(4): 737-753. 
Sarica, Y. and C. Ertekin (1985). "Descending lumbosacral cord potentials (DLCP) evoked by stimulation of the median nerve." Brain Res 325(1-2): 299-301.

Sasada, S., T. Tazoe, T. Nakajima, G. Futatsubashi, H. Ohtsuka, S. Suzuki, E. P. Zehr and T. Komiyama (2016). "A common neural element receiving rhythmic arm and leg activity as assessed by reflex modulation in arm

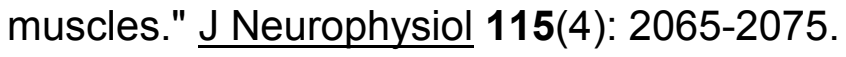

Sayenko, D. G., D. A. Atkinson, C. J. Dy, K. M. Gurley, V. L. Smith, C. Angeli, S. J. Harkema, V. R. Edgerton and Y. P. Gerasimenko (2015). "Spinal segment-specific transcutaneous stimulation differentially shapes activation pattern among motor pools in humans." J Appl Physiol (1985) 118(11): 1364-1374.

Schomburg, E. D., H. M. Meinck, J. Haustein and J. Roesler (1978). "Functional organization of the spinal reflex pathways from forelimb afferents to hindlimb motoneurones in the cat." Brain Res 139(1): 21-33.

Sharrard, W. J. (1964). "THE SEGMENTAL INNERVATION OF THE LOWER LIMB MUSCLES IN MAN." Ann R Coll Surg Engl 35: 106-122.

Sherwood, A. M., M. R. Dimitrijevic and W. B. McKay (1992). "Evidence of subclinical brain influence in clinically complete spinal cord injury: discomplete SCI." J Neurol Sci 110(1-2): 90-98.

Sherwood, A. M., W. B. McKay and M. R. Dimitrijevic (1996). "Motor control after spinal cord injury: assessment using surface EMG." Muscle Nerve 19(8): 966-979. 
Shinoda, Y., Y. Sugiuchi, Y. Izawa and Y. Hata (2006). "Long descending motor tract axons and their control of neck and axial muscles." Prog Brain Res 151: $527-563$.

Skinner, R. D., R. Nelson, M. Griebel and E. Garcia-Rill (1989). "Ascending projections of long descending propriospinal tract (LDPT) neurons." Brain Res Bull 22(2): 253-258.

Squair, J. W., A. Bjerkefors, J. T. Inglis, T. Lam and M. G. Carpenter (2016). "Cortical and vestibular stimulation reveal preserved descending motor pathways in individuals with motor-complete spinal cord injury." $\underline{\mathrm{J} \text { Rehabil }}$ Med.

Suzuki, S., T. Nakajima, G. Futatsubashi, R. A. Mezzarane, H. Ohtsuka, Y. Ohki, E. P. Zehr and T. Komiyama (2016). "Soleus Hoffmann reflex amplitudes are specifically modulated by cutaneous inputs from the arms and opposite leg during walking but not standing." Exp Brain Res.

Suzuki, S., T. Nakajima, R. A. Mezzarane, H. Ohtsuka, G. Futatsubashi and T. Komiyama (2014). "Differential regulation of crossed cutaneous effects on the soleus H-reflex during standing and walking in humans." Exp Brain Res 232(10): 3069-3078.

Troni, W., A. Di Sapio, E. Berra, S. Duca, A. Merola, F. Sperli and A. Bertolotto (2011). "A methodological reappraisal of non invasive high voltage electrical stimulation of lumbosacral nerve roots." Clin Neurophysiol 122(10): 2071-2080. 
van den Brand, R., J. Heutschi, Q. Barraud, J. DiGiovanna, K. Bartholdi, M. Huerlimann, L. Friedli, I. Vollenweider, E. M. Moraud, S. Duis, N. Dominici, S. Micera, P. Musienko and G. Courtine (2012). "Restoring voluntary control of locomotion after paralyzing spinal cord injury." Science 336(6085): 1182-1185.

Verburgh, C. A., H. G. Kuypers, J. Voogd and H. P. Stevens (1989). "Spinocerebellar neurons and propriospinal neurons in the cervical spinal cord: a fluorescent double-labeling study in the rat and the cat." Exp Brain Res 75(1): 73-82.

Wolfe, D. L., K. C. Hayes, P. J. Potter and G. A. Delaney (1996). "Conditioning lower limb H-reflexes by transcranial magnetic stimulation of motor cortex reveals preserved innervation in SCI patients." J Neurotrauma 13(6): 281291.

Zaaimi, B., S. A. Edgley, D. S. Soteropoulos and S. N. Baker (2012). "Changes in descending motor pathway connectivity after corticospinal tract lesion in macaque monkey." Brain 135(Pt 7): 2277-2289.

Zaporozhets, E., K. C. Cowley and B. J. Schmidt (2006). "Propriospinal neurons contribute to bulbospinal transmission of the locomotor command signal in

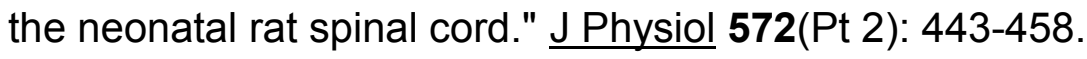

Zaporozhets, E., K. C. Cowley and B. J. Schmidt (2011). "Neurochemical excitation of propriospinal neurons facilitates locomotor command signal transmission in the lesioned spinal cord." J Neurophysiol 105(6): 28182829. 
Zehr, E. P. (2002). "Considerations for use of the Hoffmann reflex in exercise studies." Eur J Appl Physiol 86(6): 455-468.

Zehr, E. P., D. F. Collins and R. Chua (2001). "Human interlimb reflexes evoked by electrical stimulation of cutaneous nerves innervating the hand and foot." Exp Brain Res 140(4): 495-504.

Zehr, E. P., A. Frigon, N. Hoogenboom and D. F. Collins (2004). "Facilitation of soleus H-reflex amplitude evoked by cutaneous nerve stimulation at the wrist is not suppressed by rhythmic arm movement." Exp Brain Res 159(3): 382-388. 
CURRICULUM VITAE

Darryn Atkinson, MPT

\author{
4214 Hewitt Ave \\ Louisville KY 40220 \\ darrynatkinson@catholichealth.net \\ c: $832-693-9828$
}

\title{
Present Position:
}

Graduate PhD candidate

Anatomical Sciences and Neurobiology

University of Louisville, KY

Expected Graduation: October 2016

\section{Education:}

2013 Masters of Science, Anatomical Sciences and Neurobiology Dept.

University of Louisville, Louisville, KY

2005 Masters of Physical Therapy

University of Texas Southwestern Medical Center, Dallas, Texas

2003 Bachelor of Science in Exercise Science 
Harding University, Searcy, AR

\section{Professional experience:}

2016- $\quad$ Senior Instructor, Introduction to Locomotor Training continuing education course, Neurorecovery Training Institute

2012-2015 PRN Physical Therapist, Inpatient and Outpatient Rehabilitation Frazier Rehabilitation Institute Louisville, KY

2013-2015 Instructor, health and sports sciences program, college of education and human development, University of Louisville Louisville, KY

2005-2010 TIRR Memorial Hermann Hospital Houston, Texas Senior Physical Therapist, inpatient spinal cord injury rehabilitation

\section{Additional certifications:}

2007 Clinical Instructor Certification

\section{Licensure:}

2005-2012 Texas Physical Therapy license \# 1162793

2011-present Kentucky Physical Therapy license \# 005746

\section{Publications:}


2015 Sayenko DG, Atkinson DA, Floyd TC, Gorodnichev RM, Moshonkina TR, Harkema SJ, Edgerton VR, Gerasimenko YP. Effects of paired transcutaneous electrical stimulation delivered at single and dual sites over lumbosacral spinal cord. Neurosci Lett. 4;609:229-234 Nov 2015

2015 Sayenko DG, Atkinson DA, DY C, Gurley KM, Smith VL, Angeli CA, Harkema SJ, Edgerton VR, Gerasimenko, YP. Spinal segment-specific transcutaneous stimulation differentially shapes activation pattern among motor pools in humans. J Appl Physiol Epub ahead of print Mar 26, 2015

2014 Caudle KL, Atkinson DA, Brown EH, Donaldson K, Seibt E,Chea T,Smith E, Chung K, Shum-sui A, Cron CC, Magnuson DS. Hindlimb stretching alters locomotor function after spinal cord injury in adult rats. Neurorehabil Neural Repair Epub ahead of print Aug 8, 2014

2012 Behrman AL, Ardolino E, VanHiel LR, Kern M, Atkinson D, Lorenz DJ, Harkema SJ. Assessment of Functional Improvement Without Compensation Reduces Variability of Outcome Measures After Human Spinal Cord Injury. Arch Phys Med Rehabil 93:1518-1529, 2012

Ullrich PM, Spungen AM, Atkinson D, Bombardier CH, Chen Y, Erosa NA, Groer S, Ottomanelli L, Tulsky DS. Activity and participation after spinal cord injury: State-of-the-art report. J Rehabil Res Dev. 49(1):15574, 2012. 
Li K, Atkinson D, Boakye M, Tolfo CZ, Aslan S, Green M, McKay B, Ovechkin A, Harkema SJ. Quantitative and sensitive assessment of neurophysiological status after human spinal cord injury. J Neurosurg Spine (Suppl) 17:77-86, 2012

\section{Presentations:}

2014 Effects of ulnar nerve stimulation on excitability of lumbosacral motor neuron pools: method of investigation of long propriospinal pathways. 20th Annual Kentucky Spinal Cord and Head Injury Research Trust (KSCHIRT) Symposium, Lexington, KY

2010 The Thoraco-Lumbar Control Scale. NeuroRecovery Network National Summit, Louisville KY

2009 The Thoraco-lumbar Control Scale (as part of the symposium "Developing an International Data Set for SCl) American Congress of Rehabilitation Medicine, Denver CO

\section{Abstracts:}

2016 Atkinson DA, Harkema SJ, Trimble SA, Mendez L, Behrman AL. Functional neurophysiological assessment of volitional motor control following pediatric spinal cord injury. Society for Neuroscience Annual Conference, San Diego, CA. 
Behrman AL, Trimble SA, Alvarado L, Atkinson DA. Task-dependent recruitment of spinal motor pools after pediatric spinal cord injury. Society for Neuroscience Annual Conference, San Diego, CA.

Trimble SA, Atkinson DA, Behrman AL. Unexpected stepping gains in a young child with a functionally isolated spinal cord. Society for Neuroscience Annual Conference, San Diego, CA.

2015 Atkinson DA, Sayenko DG, Mink AM, Gurley KM, Smith V, Gerasimenko YP, Harkema SJ. Identifying descending propriospinal influence on lumbosacral motor neuron excitability after spinal cord injury: Effects of ulnar nerve stimulation on MMR amplitude in leg muscles. Society for Neuroscience Annual Conference, Chicago, II.

Mink AM, Sayenko DG, Atkinson DA, Gerasimenko YP, Harkema SJ. Identifying supraspinal influences on lumbosacral motor neuron excitability after spinal cord injury: Effects of galvanic vestibular stimulation and acoustic startle reflex on MMR amplitude in leg muscles. Society for Neuroscience Annual Conference, Chicago, II.

2013 Atkinson DA, Sayenko DG, Gurley KM, Smith V, Ferreira CK, Angeli CA, Harkema SJ Modulation of multi-segmental monosynaptic responses from leg muscles by electrical excitation of the ulnar nerve: Investigation of long descending propriospinal pathways. Society for Neuroscience Annual Conference, San Diego, CA. Sayenko DG, Atkinson DA, Dy C, Rejc E, Gurley K, Smith V, Ferreira C, Angeli CA, Edgerton VR, Gerasimenko Y, Harkema SJ Location-specific 
effects of transcutaneous lumbar spinal electrical stimulation on the recruitment of proximal and distal leg muscles in healthy individuals. Society for Neuroscience Annual Conference, San Diego, CA.

2012 Atkinson D, Aslan S, Tolfo CZ, Green M, McKay B, Ovechkin A, Harkema SJ. Quantitative Assessment of Neurophysiological Status after Spinal Cord Injury. Academy for Spinal Cord Injury Professionals, Annual Conference, Las Vegas, NV.

Ward P, Shah CN, Herrity AN, Atkinson DA, Stewart BR, Harkema SJ, and Hubscher $\mathrm{CH}$. Step training improves overground locomotion following moderate contusion in rats: open field scoring, kinematics, and gait analysis. Society for Neuroscience Annual Conference, New Orleans, LA.

Terson de Paleville DG, Atkinson DA, Chopra M, Lorenz DJ, Tolfo CZ, Harkema SJ. Functional Neurophysiological Assessment of the Trunk Muscles in individuals with Spinal Cord Injury. Society for Neuroscience Annual Conference, New Orleans, LA.

2011 Caudle KL, Atkinson DA, Brown EH, Chea T, Smith E, Chung K, Shum Siu A, Morehouse J, Burke D, Magnuson DSK. The effect of hindlimb stretch on locomotor recovery after contusive $\mathrm{SCl}$ in the rat. Society for Neuroscience Annual Conference, Washington, DC.

2010 Atkinson, D and Graves, DE. Interrelation of Trunk Scale and Functional Outcome Measures for Spinal Cord Injury $4^{\text {th }}$ National (Canadian) Spinal Cord Injury Conference, Niagara Falls, Ontario, Canada. 
Atkinson,D and Graves, DE. Reliability and Sensitivity of the ThoracoLumbar Control Scale. American Spinal Cord Injury Professionals (ASCIPRO) annual meeting, Las Vegas, NV

2009 Atkinson, D and Graves, DE. Scale Structure of the Thoracolumbar Control Scale American Spinal Injury Association Annual Conference, Dallas, Texas.

2008 Atkinson D, Atkinson K, Kern M, Hale J, Feltz M, Graves DE. Reliability of a Thoracic-Lumbar Control Scale for Use in Spinal Cord Injury Research National Spinal Cord Injury Conference \& Interurban Spinal Cord Injury Conference, Toronto, Canada

Atkinson D, Atkinson K, Kern M, Hale J, Feltz M, Graves DE. Reliability of a Thoracic-Lumbar Control Scale for Use in Spinal Cord Injury Research American Congress of Rehabilitation Medicine and the American Society of Neurorehabilitation Joint Educational Conference, Toronto, Canada

Abbott R, Atkinson D, Atkinson K, Hyde L, Kern M, Marquart M, Seale J, White C, Graves D, Latorre J. Comparing Gait Outcome Measures: the $\underline{\text { Role of the GAITRite }{ }^{\circledR} \text { Walkway System in Assessing Functional }}$ Ambulators with Spinal Cord Injury. Combined Sections Meeting of the APTA, Nashville, Tennessee

2007 Abbott R, Atkinson D, Atkinson K, Hyde L, Kern M, Marquart M, Seale J, White C, Graves D, Latorre J. Comparing Gait Outcome Measures: the Role of the GAITRite ${ }^{\circledR}$ Walkway System in Assessing Functional 
Ambulators with Spinal Cord Injury. American Spinal Injury Association, Tampa, Florida.

\section{Teaching Experience:}

2016 Introduction to Locomotor Training Senior Instructor. Organize and lead a team of physical therapists and rehab technicians in putting on a 2.5 day continuing education course teaching the physical skills and scientific framework for the implementation of standardized body-weight support treadmill training for recovery of neurological function after spinal cord injury at neurorehabilitation sites within the Neurorecovery network.

2013-2015 Human Biomechanics, University of Louisville, Louisville, Kentucky 2010 TIRR neurologic PT residency program module instructor:

- Shoulder dysfunction following SCI: assessment and intervention

- SCl transfers: assessment and training (emphasis on tetraplegic transfer analysis and training)

TIRR Memorial Hermann, Houston, Texas

2007-2010 Spinal Cord Injury Functional Mobility Lab, Texas Woman's University (DPT program), TIRR Memorial Hermann, Houston, Texas

SCI Mobility Labs- Ventilators, Bathroom Equipment and Mechanical Lift Transfers, University of Texas Medical Branch at 
Galveston (DPT program), TIRR Memorial Hermann, Houston, Texas

NeuroRecovery Network (NRN) Regional Training course (Lecture and lab components) TIRR Memorial Hermann, Houston, Texas 